Aus der Klinik für Neurochirurgie

(Prof. Dr. med. V. Rohde)

der Medizinischen Fakultät der Universität Göttingen

\title{
Der Einfluss des temporären Clippings auf die Häufigkeit zerebraler Vasospasmen nach aneurysmatischer Subarachnoidalblutung
}

\author{
INAUGURAL-DISSERTATION \\ zur Erlangung des Doktorgrades \\ für Zahnheilkunde \\ der Medizinischen Fakultät der \\ Georg-August-Universität zu Göttingen
}

\author{
vorgelegt von \\ Martin Voit \\ aus Hagen
}

Göttingen 2017 
Referentin:

Prof. Dr. med. D. Mielke

Ko-Referent:

Prof. Dr. med. M. Knauth

Drittreferent:

Datum der mündlichen Prüfung: 28. Juni 2017 
Hiermit erkläre ich, die Dissertation mit dem Titel

"Der Einfluss des temporären Clippings auf die Häufigkeit zerebraler Vasospasmen nach aneurysmatischer Subarachnoidalblutung" eigenständig angefertigt und keine anderen als die von mir angegebenen Quellen und Hilfsmittel verwendet zu haben.

Göttingen, den 01.06.2017

(Unterschrift) 


\section{Inhaltsverzeichnis}

Inhaltsverzeichnis I

Abbildungsverzeichnis III

Tabellenverzeichnis III

Abkürzungsverzeichnis IV

$1 \quad$ Einleitung $\quad 1$

1.1 Das Krankheitsbild der Subarachnoidalblutung .......................................... 1

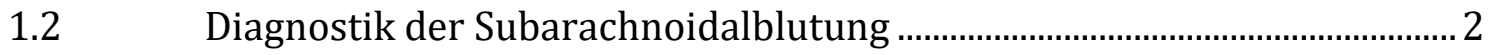

1.3 Therapie der Subarachnoidalblutung................................................................ 4

1.3.1 Neurochirurgischer Aneurysma-Verschluss: Clipping ................................. 4

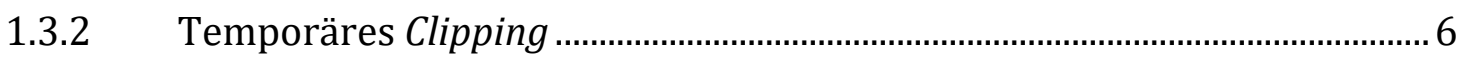

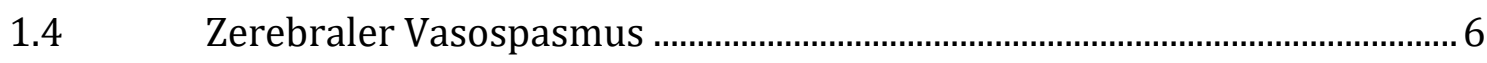

1.5 Gegenstand und Zielsetzung dieser Arbeit ...................................................... 7

$2 \quad$ Material und Methoden $\quad 8$

2.1 Studiendesign und Patientenkollektiv ....................................................... 8

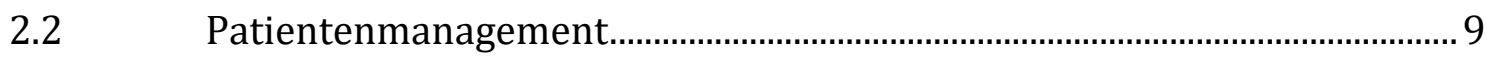

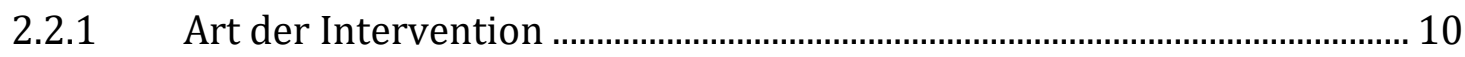

2.3 Ausgewertete bildgebende Verfahren ............................................................. 10

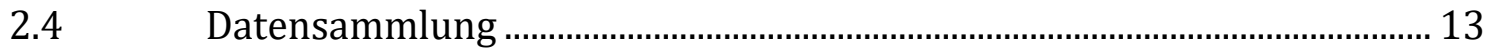

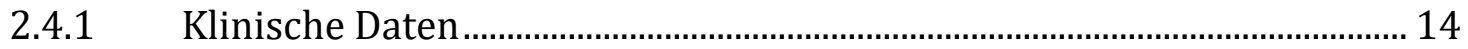

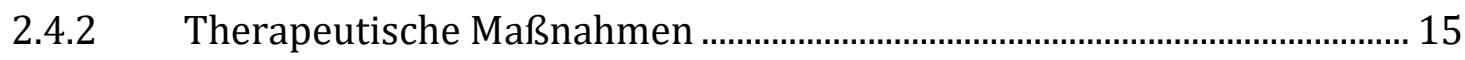

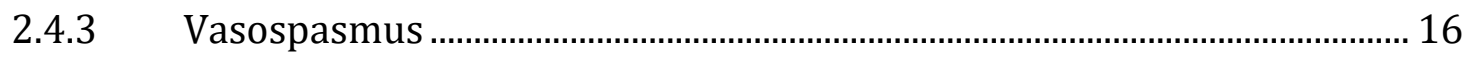

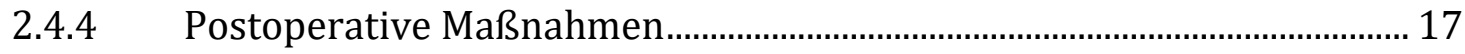

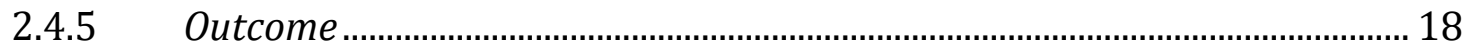

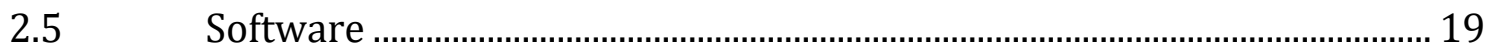

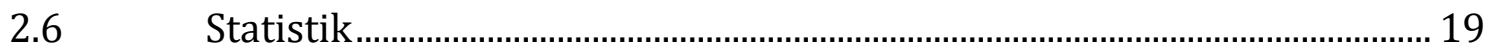

$\begin{array}{llr}3 & \text { Ergebnisse } & 21\end{array}$

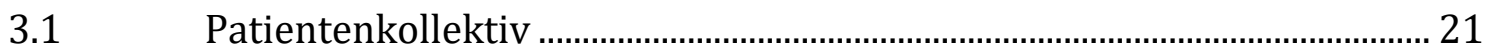




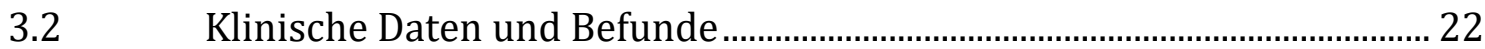

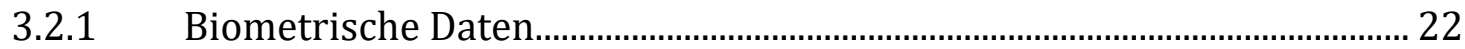

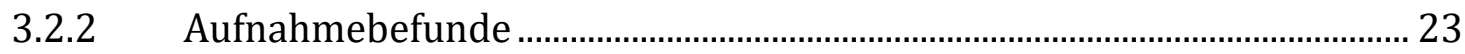

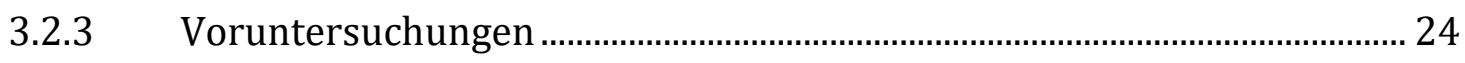

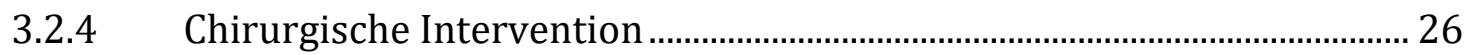

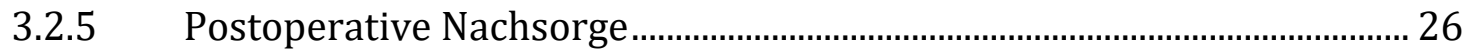

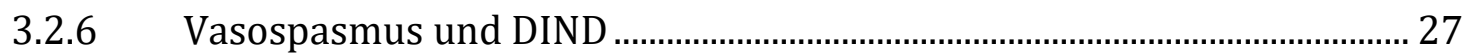

3.2.7 Temporäres Clipping …................................................................................... 28

3.2.8 Multivariate Analyse.................................................................................. 31

$4 \quad$ Diskussion $\quad 33$

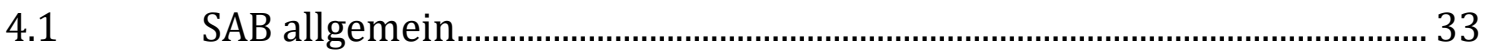

4.1.1 Anatomische Grundlagen ............................................................................ 34

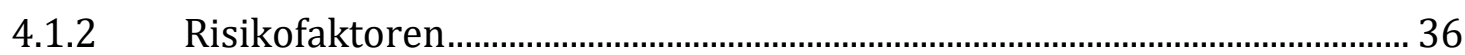

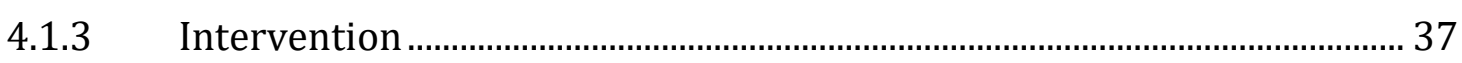

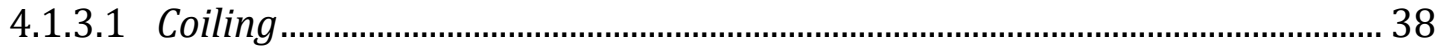

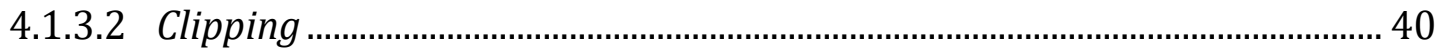

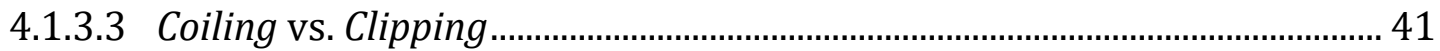

4.2 Temporäres Gefäß-Clipping ……................................................................ 44

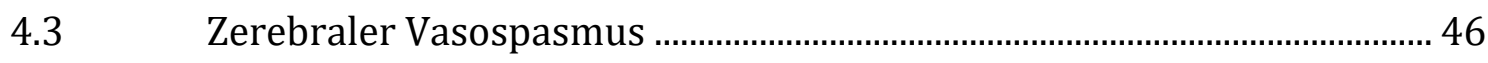

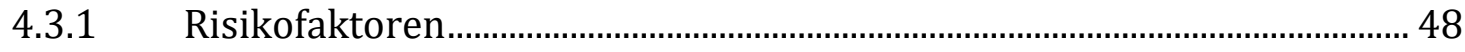

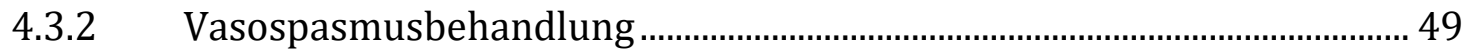

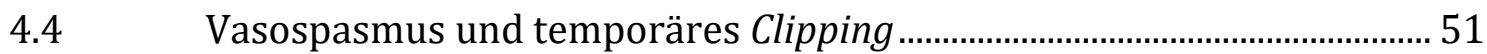

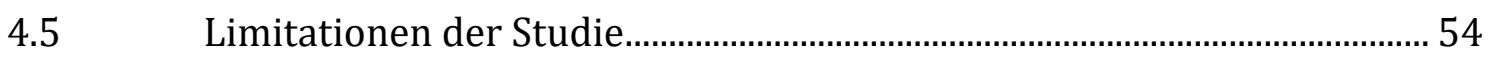

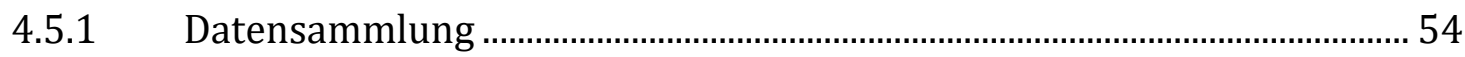

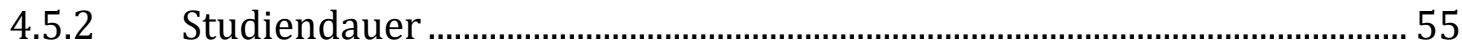

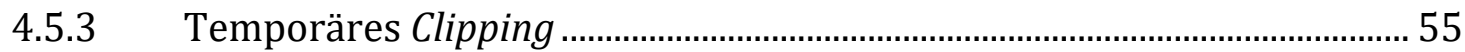

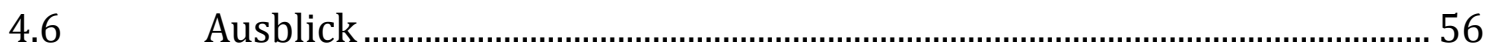

$\begin{array}{lll}5 & \text { Zusammenfassung } & 57\end{array}$

$6 \quad$ Literaturverzeichnis $\quad 59$ 


\section{Abbildungsverzeichnis}

Abbildung 1-1: Schematische Darstellung des Circulus arteriosus Willisi .......................................................

Abbildung 1-2: Diagnostik einer aSAB . .........................................................................................................

Abbildung 1-3: Intraoperative Aufnahmen einer pterionalen Kraniotomie....................................................

Abbildung 2-1: Axiale cCT-Aufnahmen mit Nachweis von aSABs verschiedener Fisher-Grade........... 11

Abbildung 2-2: DSA-Aufnahme eines ACI-Aneurysmas........................................................................................ 12

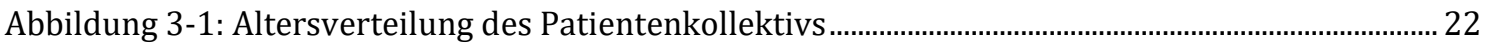

Abbildung 3-2: Patientenverteilung nach Hunt und Hess ………………………………………………….. 23

Abbildung 3-3: Patientenverteilung nach Glasgow Coma Scale …………………………………………... 24

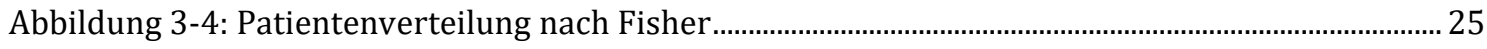

Abbildung 3-5: Patientenverteilung nach Glasgow Outcome Scale .................................................................. 27

Abbildung 3-6: Vergleich des GOS bei Patienten mit und ohne temporäres Clipping.............................. 29

Abbildung 3-7: DIND-Aufkommen bei Patienten mit und ohne temporäres Clipping............................... 29

Abbildung 3-8: Aufkommen Spasmus-induzierter Infarkte bei Patienten mit und ohne

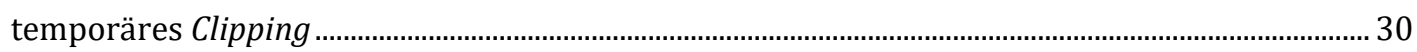

Abbildung 3-9: Vasospasmus-Aufkommen bei Patienten mit und ohne temporäres Clipping ............. 30

Abbildung 4-1: Anordnung der Hirnhäute und deren Räume...................................................................... 35

Abbildung 4-2: Schematische Darstellung eines Aneurysma-Verschlusses mittels Coiling.................... 39

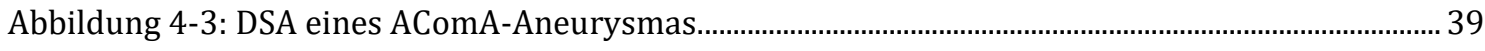

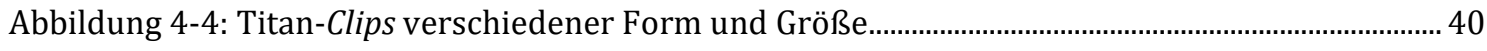

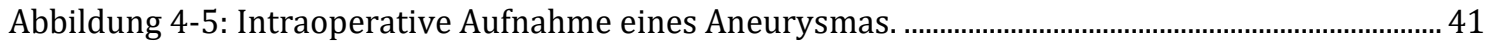

Abbildung 4-6: Zeichnerische Darstellung eines temporären Clippings....................................................... 45

\section{Tabellenverzeichnis}

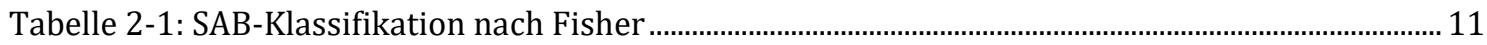

Tabelle 2-2: Glasgow Coma Scale nach Teasdale und Jennett ........................................................................... 14

Tabelle 2-3: SAB-Klassifikation nach Hunt und Hess ........................................................................................ 15

Tabelle 2-4: Glasgow Outcome Scale nach Jennett und Bond …………………………………………...... 18

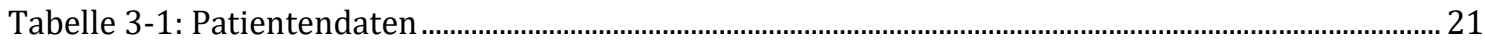

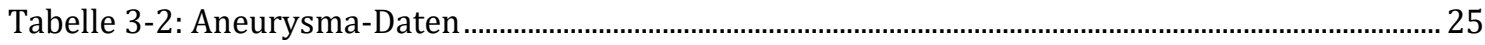

Tabelle 3-3: Logistische Regressionsanalyse der angegebenen Parameter in Bezug auf das

Vorliegen eines Vasospasmus.................................................................................................................. 32 


\section{Abkürzungsverzeichnis}

A./Aa.

ACA

AChoA

ACI

AComA

AICA

aSAB

BA

BRAT

cCT

CTA

DIND

DSA

EVD

GCS

GOS

$\mathrm{H} \& \mathrm{H}$

ICB

ICG

ISAT

KI

LD

MCA
Arteria/Arteriae

Arteria cerebri anterior

Arteria choroidea anterior

Arteria carotis interna

Arteria communicans anterior

Arteria cerebelli inferior anterior

aneurysmatische Subarachnoidalblutung

Arteria basilaris

The Barrow Ruptured Aneurysm Trial

kraniale Computertomographie

computertomographische Angiographie

Delayed Ischaemic Neurological Deficit

digitale Subtraktionsangiographie

externe Ventrikeldrainage

Glasgow Coma Scale

Glasgow Outcome Scale

Hunt und Hess-Grad

intrazerebrale Blutung

Indozyaningrün-Fluoreszenz

International Subarachnoid Aneurysm Trial

Konfidenz-Intervall

Lumbaldrainage

Arteria cerebri media 
MRT

$\mathrm{OA}$

OR

PCA

PComA

PICA

SAB

SAH

SUCA

TCD

tSAB

UMG

VA

WFNS
Magnetresonanztomographie

Arteria ophtalmica

Odds Ratio

Arteria cerebri posterior

Arteria communicans posterior

Arteria cerebelli inferior posterior

Subarachnoidalblutung

Subarachnoid Haemorrhage

Arteria cerebelli superior

transkranielle Dopplersonographie

traumatische Subarachnoidalblutung

Universitätsmedizin Göttingen

Arteria vertebralis

World Federation of Neurosurgical Societies (SAH Grading Scale) 


\section{Einleitung}

\subsection{Das Krankheitsbild der Subarachnoidalblutung}

Die Subarachnoidalblutung (SAB) ist ein schwerwiegendes neurologisches Krankheitsbild, bei dem es zum Austritt von Blut in den das Gehirn umgebenden liquorgefüllten Subarachnoidalraum kommt.

Die klassischen Symptome dieser Erkrankung sind plötzlich eintretende, stärkste, vernichtende Kopfschmerzen, häufig im Zusammenhang mit Übelkeit und Erbrechen, Meningismus und Bewusstseinseintrübung (Edlow und Caplan 2000; Linn et al. 1998). Zusätzlich können epileptische Anfälle, Paresen oder Sprachstörungen auftreten.

Die SAB gehört zu den Unterformen des Schlaganfalls, macht etwa 2 - $5 \%$ aller Schlaganfälle aus (Sudlow und Warlow 1997; van Gijn et al. 2007) und geht mit einer sehr hohen Morbidität und Mortalität einher. Sie weist eine Inzidenz von ca. 10/100.000 Personen/Jahr auf (Linn et al. 1996; Pobereskin 2001). Etwa die Hälfte aller Patienten, die eine SAB erleiden, überlebt die Erkrankung nicht, wobei ca. $10 \%$ bereits versterben, bevor eine medizinische Versorgung möglich ist. Im Falle des Überlebens leidet etwa ein Drittel der Patienten unter anhaltenden, teils schwerwiegenden neurologischen Schäden und Behinderungen, die eine dauerhafte pflegerische Versorgung nötig machen (Suarez et al. 2006).

Die häufigste Ursache für eine Subarachnoidalblutung liegt in der Ruptur eines Aneurysmas der intrakraniellen basalen Arterien, die für die Blutversorgung des Gehirns zuständig sind. Dies betrifft ca. 85 \% aller Subarachnoidalblutungen, weitere Fälle sind unter anderem die milde verlaufende nicht-aneurysmatische, perimesenzephale Blutung, die traumatische SAB (tSAB) bei Unfällen mit Schädel-Hirn-Trauma oder arterio-venöse Malformationen (van Gijn et al. 2007; van Gijn und Rinkel 2001). In unserer Studie betrachteten wir ausschließlich Patienten mit nachgewiesener aneurysmatischer SAB.

In den meisten Fällen treten die Aneurysmen am Circulus arteriosus Willisi auf, der an der Hirnbasis liegt und die großen, paarig angelegten Arterien des Gehirns zu einem Kreislauf verbindet (s. Abbildung 1-1). Diese Gefäße und vor allem ihre Verzweigungen (Bifurkationen) sind bevorzugte Stellen für die Ausbildung von Aussackungen der Gefäßwände, sogenannten Aneurysmen. 


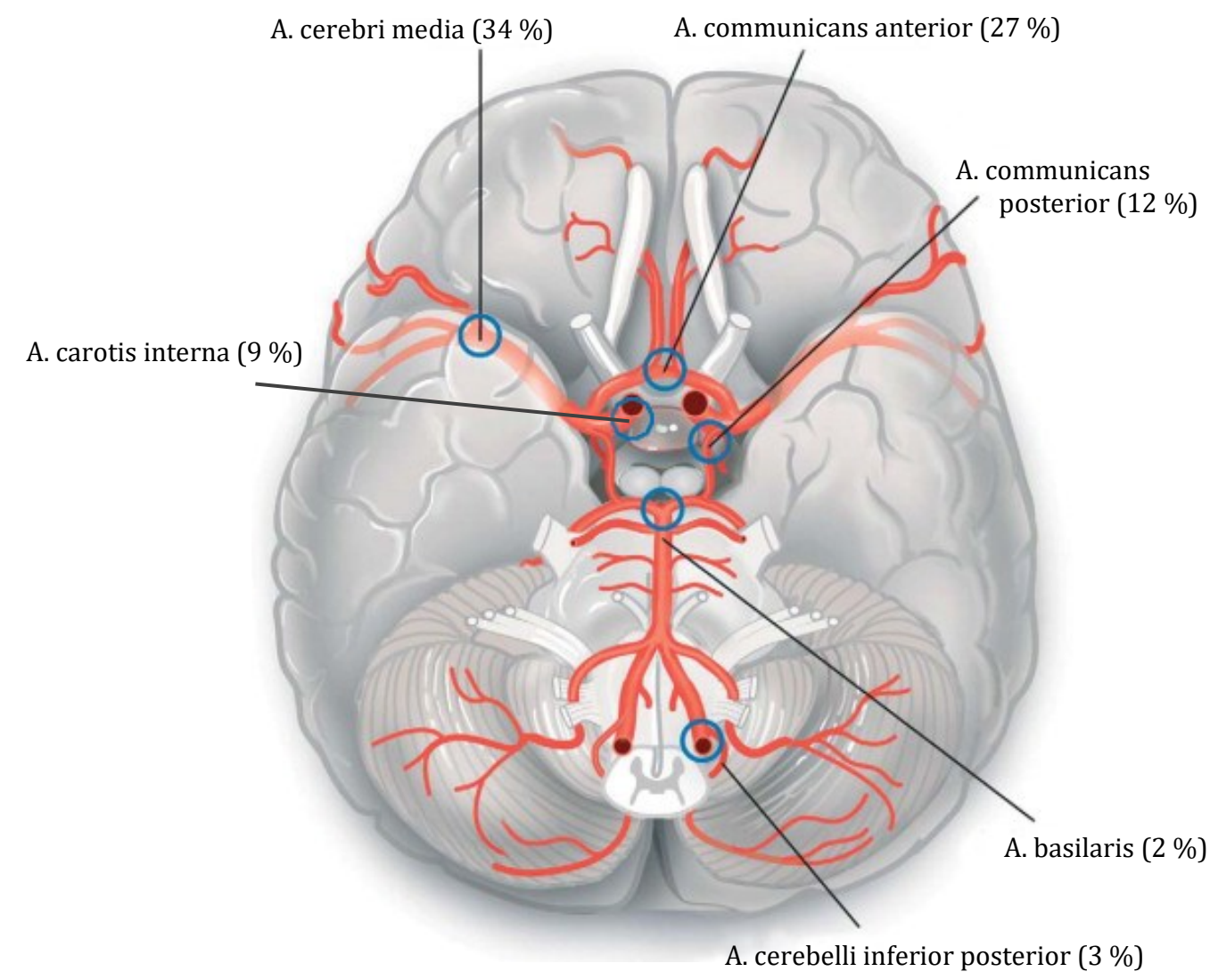

Abbildung 1-1: Schematische Darstellung des Circulus arteriosus Willisi

Angaben der häufigsten Aneurysma-Lokalisationen dieser Untersuchung in \% (modifiziert entnommen aus van Gijn et al. 2007; die Verwendung erfolgt mit Genehmigung des Elsevier-Verlags).

Bei der Ruptur eines Aneurysmas kommt es zum Austritt von Blut in den umgebenden Subarachnoidalraum. Im Zuge einer Subarachnoidalblutung kann es über Verbindungen zum inneren Liquorraum auch zu Bluteintritt in das Ventrikelsystem oder zu Einblutungen ins Hirnparenchym (intrazerebrale Blutung = ICB) kommen. Verbunden hiermit sind mögliche neurologische Ausfallerscheinungen in Abhängigkeit vom betroffenen Hirnareal.

\subsection{Diagnostik der Subarachnoidalblutung}

Liegt eine aneurysmatische Subarachnoidalblutung ( $\mathrm{aSAB}$ ) vor, ist eine umgehende medizinische Intervention angezeigt. $\mathrm{Um}$ das Risiko postinterventioneller Komplikationen möglichst gering zu halten, wird eine Versorgung des Aneurysmas innerhalb der ersten 72 Stunden nach Eintreten der Blutung empfohlen (Kassell et al. 1990b). In erster Linie erfolgt eine umfassende Diagnostik, um das Ausmaß und die Lokalisation der Blutung festzustellen. Dies 
geschieht primär durch präoperative kraniale Computertomographien (cCT, s. Abbildung 1-2 A) und CT-Angiographie-Untersuchungen (CTA, s. Abbildung 1-2 B).

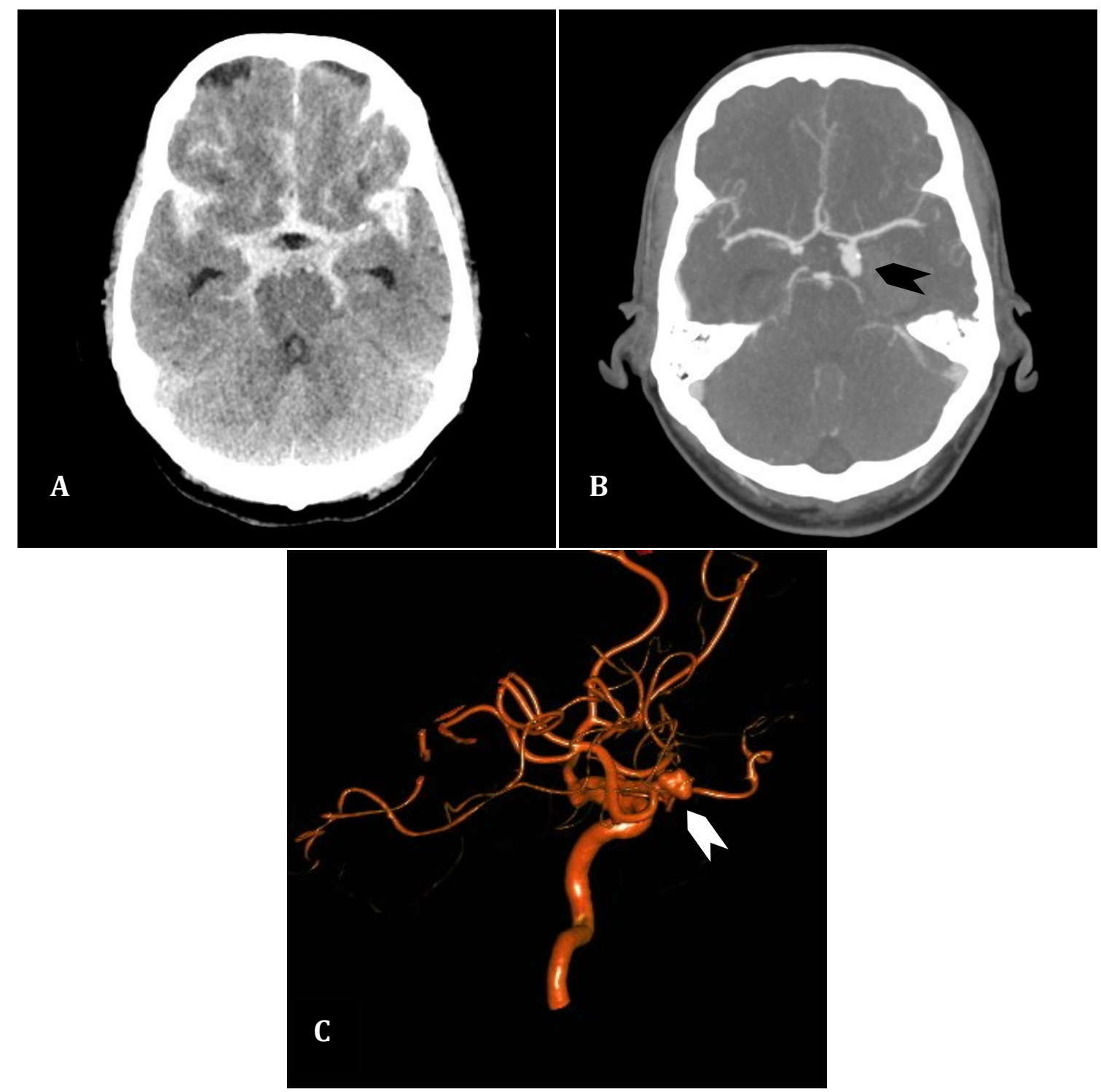

Abbildung 1-2: Diagnostik einer aSAB.

[A] Präoperative axiale cCT-Aufnahme mit Nachweis einer subarachnoidalen Blutansammlung. [B] Nachweis eines Aneurysmas an der ACI links mittels axialer CTA-Aufnahme (s. Pfeil). [C] 3-D-DSA eines Aneurysmas an der MCA rechts (s. Pfeil). (Zur Verfügung gestellt vom Institut für Diagnostische und Interventionelle Neuroradiologie, Universitätsmedizin Göttingen.)

Die daraus gewonnenen Erkenntnisse führen zu einem genauen Bild der von der Blutung betroffenen Gebiete und der Lage und Größe des Aneurysmas, dessen Ruptur die Blutung ausgelöst hat. Falls die radiologische Diagnostik keinen Nachweis einer SAB zeigt, jedoch der dringende klinische Verdacht besteht, ist die Durchführung einer Lumbalpunktion indiziert, um eine eventuelle Blutung durch 
Nachweis im Liquor zu bestätigen bzw. endgültig auszuschließen. Hierbei kann auch eine 3-Gläser-Probe hergestellt werden, die eine Differenzierung zwischen einer artifiziellen, durch die Punktion bedingten Liquoreinblutung (iatrogene Blutung) oder einer stattgehabten SAB erlaubt.

\subsection{Therapie der Subarachnoidalblutung}

In Abhängigkeit von Konfiguration und Lage des Aneurysmas sowie dem klinischen Zustand des Patienten wird entschieden, ob eine mikrochirurgische oder endovaskuläre Therapie durchgeführt wird, um das Aneurysma zu verschließen und die Blutung zu stillen. Bis Mitte der 1990er Jahre stand lediglich die chirurgische Therapie (Clipping) zur Verfügung, bevor die von Guglielmi entwickelte Methode, Aneurysmen interventionell durch einen Katheter mittels Drahtschlingen zu verschließen (Coiling), breitere Anwendung fand (Guglielmi et al. 1991).

\subsubsection{Neurochirurgischer Aneurysma-Verschluss: Clipping}

Im Gegensatz zur endovaskulären Therapie ist bei der chirurgischen Intervention zum Aneurysma-Verschluss eine Eröffnung der Schädeldecke (Kraniotomie) erforderlich, um einen direkten Zugang zum betroffenen Gefäß und zum rupturierten Aneurysma zu ermöglichen. Unter Schonung des Hirngewebes wird nach erfolgter Lokalisation in der cCT das Aneurysma dargestellt. In der Folge wird eine Titan-Klemme - ein sogenannter Clip - um den Hals des Aneurysmas platziert. Dieses Clipping des Aneurysmas sorgt dafür, dass die Aussackung an der Gefäßwand verschlossen wird und somit keine Blutversorgung des Aneurysmas mehr möglich ist. Der arterielle Blutfluss wird nicht mehr durch das Ausfließen von Blut in das Aneurysma gestört und es wird weiterhin verhindert, dass durch die rupturierte Aneurysmawand weiteres Blut in den Subarachnoidalraum gelangt. Je nach Lokalisation des Aneurysmas werden verschiedene Kraniotomien gewählt. Aneurysmen, die an Arterien im anterioren Stromgebiet liegen (Arteria cerebri media [MCA], A. communicans anterior [AComA], A. communicans posterior [PComA], A. carotis interna [ACI], A. cerebri anterior [ACA], A. ophtalmica [OA]), werden über einen pterionalen Zugang aufgesucht (s. Abbildung 1-3). Für die 
Arterien des posterioren Stromgebietes (A. cerebelli inferior posterior [PICA], $A$. cerebelli superior [SUCA], A. cerebelli inferior anterior [AICA]) wird ein suboccipitaler Zugang gewählt. Aneurysmen an $\operatorname{der} A$. basilaris (BA) und $A$. vertebralis (VA) können je nach Lage am Gefäß auch über einen pterionalen, an der PICA ggf. über einen transkondylären Zugang operiert werden. Aneurysmen an der A. pericallosa werden über eine interhemisphärische Kraniotomie versorgt.
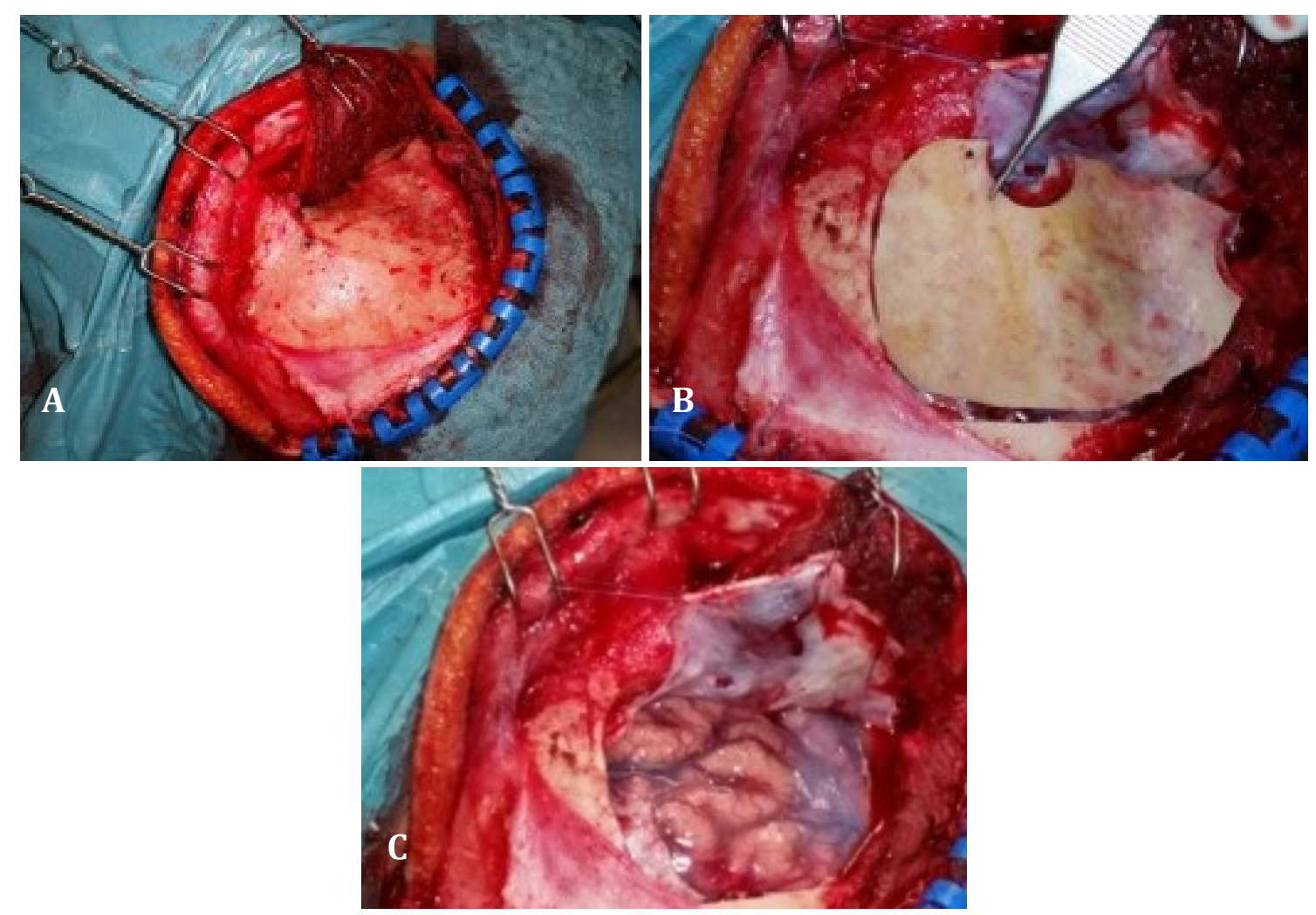

Abbildung 1-3: Intraoperative Aufnahmen einer pterionalen Kraniotomie.

[A] Darstellung der Schädeldecke. [B] Eröffnung der Schädeldecke. [C] Zugang zum Hirngewebe. (Zur Verfügung gestellt von Prof. Dr. D. Mielke, Klinik für Neurochirurgie, UMG.)

Nach Platzierung des Clips wird intraoperativ per Mikrodoppler-Sonographie und/oder Indozyaningrün-Fluoreszenz-Angiographie (ICG) kontrolliert, dass keine Störung der Durchblutung durch akzidentellen Verschluss von beteiligten Gefäßen vorliegt und außerdem eine komplette Aneurysmaausschaltung ohne Restperfusion besteht (Siasios et al. 2012). 


\subsubsection{Temporäres Clipping}

Eine zusätzliche operative Interventionsmöglichkeit bei der mikrochirurgischen Versorgung rupturierter Aneurysmen stellt der temporäre Verschluss der zuführenden Gefäße dar. Nach Freilegung des Aneurysmas wird das versorgende Gefäß durch einen proximal (und eventuell zusätzlich distal) des Aneurysmas angelegten Clip so im Blutfluss eingeschränkt, dass anschließend der nicht mehr durchblutete Aneurysmasack mit definitiven, permanenten Clips ausgeschaltet werden kann. Dadurch wird die Gefahr einer zusätzlichen, intraoperativen Blutung durch Manipulation des Aneurysmas deutlich reduziert. Außerdem ermöglicht es eine gute Gefäßrekonstruktion, beispielsweise bei breitbasigen Aneurysmen (Taylor et al. 1996). Die Entwicklung und Anwendung dieser Methode geht schon viele Jahrzehnte zurück. Erstmals berichtete Jefferson 1928 darüber und spätestens in den 1960er Jahren wurde sie zur routinemäßigen Anwendung weiterentwickelt (Pool 1961). Insbesondere ist diese Intervention erforderlich, wenn es zu einer intraoperativen Ruptur des Aneurysmas kommt, um somit die Blutung zu stoppen.

\subsection{Zerebraler Vasospasmus}

Eine schwerwiegende Komplikation im Zuge der Behandlung von Subarachnoidalblutungen ist das Auftreten zerebraler Vasospasmen. Diese sind hauptverantwortlich für bleibende neurologische Schäden und/oder den Tod der Patienten nach Behandlung einer SAB (Kassell et al. 1990a). Sie treten sowohl bei neurochirurgischem als auch bei endovaskulärem Aneurysmaverschluss gleichermaßen auf (Charpentier et al. 1999; Hoh et al. 2004). Viele verschiedene Faktoren werden als Ursache für die Entstehung von Vasospasmen angeführt, beispielsweise Bestandteile des aus dem Aneurysma stammenden Blutgerinnsels, Proteine der rupturierten Gefäßwand oder aber auch die Manipulation des Gefäßes und der umgebenden Gewebe beim Verschluss des Aneurysmas (Pluta et al. 2009; Weir 1995). Bei bis zu 70 \% der Patienten kommt es im postoperativen Verlauf zu dieser Komplikation (Crowley et al. 2011; Dorsch und King 1994; Torbey et al. 2001). Besonders gefährdet sind die Patienten innerhalb der ersten 14 Tage nach Intervention (Dorsch und King 1994; Mindea et al. 2006; van Gijn et 
al. 2007; Weir et al. 1978), weshalb vor allem in diesem Zeitraum eine intensivmedizinische Überwachung und entsprechende Untersuchungen der Patienten von großer Bedeutung sind.

\subsection{Gegenstand und Zielsetzung dieser Arbeit}

Da Vasospasmen sowohl bei Patienten mit chirurgischer als auch mit endovaskulärer Versorgung der Aneurysmen auftreten, stellt sich die Frage, inwieweit die Art des Eingriffs einen Einfluss auf das Vorkommen dieser Komplikation hat.

Trotz der mittlerweile weitverbreiteten Anwendung des temporären Clippings als Bestandteil der chirurgischen Aneurysma-Therapie gibt es zurzeit kaum wissenschaftliche Studien, die sich mit dem Einfluss und den Auswirkungen desselben auf den Zustand des Patienten während und nach der Operation beschäftigen. Aktuelle Studien zeigen, dass temporäres Clipping keinen Einfluss auf den langfristigen postoperativen Zustand und das Outcome des Patienten hat und als sichere intraoperative Gefäßmanipulation gilt (Griessenauer et al. 2014), wohl aber das Auftreten intraoperativer Aneurysmarupturen und damit verbundener Blutungen verringern kann (Dhandapani et al. 2013).

Gegenstand dieser Dissertationsarbeit ist es, einen Zusammenhang zwischen dem temporären, intraoperativen Gefäß-Clipping und einer der Hauptkomplikationen nach einer Subarachnoidalblutung, dem zerebralen Vasospasmus, zu ergründen.

Dazu wurde eine umfassende, retrospektive Datenanalyse durchgeführt, die ein Patientenkollektiv mit chirurgischer aSAB-Therapie umfasste. Das Auftreten oder Ausbleiben eines Vasospasmus in den ersten 14 Tagen nach der Operation wurde in einer statistischen Gegenüberstellung in einen Zusammenhang mit stattgehabtem oder ausgebliebenem intraoperativem, temporärem Clipping gebracht. 


\section{Material und Methoden}

\subsection{Studiendesign und Patientenkollektiv}

Die Dissertationsarbeit beruht auf der Analyse einer retrospektiven Zusammenstellung eines Patientenkollektivs mit aneurysmatischer Subarachnoidalblutung im Zeitraum von 1990 bis 2013 in einer umfassenden Datenbank. Dabei kam es zur Einbeziehung einer bereits existierenden Datenbank, in der unter Leitung von Prof. Dr. med. V. Rohde Daten von Patienten gesammelt wurden, die im Zeitraum von 1990 bis 2005 in der Neurochirurgischen Universitätsklinik der Rheinisch-Westfälischen Technischen Hochschule Aachen (RWTH Aachen) mit aneurysmatischer SAB behandelt wurden. Diese wurde uns von Prof. Dr. Rohde, seit 2005 Leiter der Abteilung für Neurochirurgie der Universitätsmedizin Göttingen (UMG), zur Verfügung gestellt. Darauf aufbauend wurde für den Zeitraum der Jahre 2005 bis 2013 von mir in Zusammenarbeit mit Frau ZÄ Patricia Suntheim eigenhändig eine an die bereits bestehende Datensammlung angelehnte neue Datenbank angelegt, die alle Patienten einschließt, die in diesem Zeitraum in der neurochirurgischen Abteilung der UMG mit aneurysmatischer SAB behandelt wurden. (Frau Suntheim bearbeitete eine von meiner Arbeit abweichende Fragestellung zu dieser Datensammlung.)

Das vorher in Aachen etablierte Behandlungskonzept für Patienten mit aSAB wurde von Prof. Rohde nach 2005 entsprechend in der UMG fortgeführt, sodass die Therapie über den gesamten beobachteten Zeitraum auf den gleichen Strategien basierte.

In die Studie nicht eingeschlossen wurden Patienten mit traumatischer SAB sowie Patienten mit SAB, bei denen kein Aneurysma nachgewiesen werden konnte. Weitere Ausschlusskriterien waren Blutungen aus arterio-venösen Malformationen und Minderjährigkeit der Patienten. Für jeden Patienten wurden der Verlauf der Erkrankung und deren stationäre und operative Behandlung nach Aktenlage aufgearbeitet und dokumentiert. Dabei wurden Behandlungs- und Anästhesieprotokolle sowie die Dokumentation der intensivmedizinischen Behandlung und Entlassungsbriefe einbezogen. Patienten, deren Datenlage so unvollständig war, dass eine Beurteilung der stationären Behandlung nicht möglich war, blieben für die Analyse nicht berücksichtigt. 
Die einzelnen in die Datenbank aufgenommenen Parameter sind im weiteren Verlauf aufgeführt und erläutert.

Die beiden Datenbanken aus Aachen und Göttingen wurden miteinander zu einer umfassenden Datensammlung verbunden und als Gesamtkollektiv aller aSABPatienten analysiert und ausgewertet. In der Folge wurden für diese Studie lediglich die Patienten ausgewählt, die durch einen operativen Eingriff mit Clipping der Aneurysmen behandelt wurden. Alle Patienten, bei denen statt des Clippings eine endovaskuläre, neuroradiologische Therapie (Coiling) durchgeführt wurde, flossen nicht in die Analyse mit ein. Ebenso ausgeschlossen wurden Patienten, die nach Eintreten der SAB gar keine Therapie erfahren haben. Insgesamt umfasst die Datenbank nach diesen Auswahlkriterien 535 Patienten.

Die Dissertationsarbeit wurde der Ethikkommission der UMG als retrospektive Studie gemeldet (Anmeldenummer: DOC_37_2013). Da die Datenerfassung der jeweiligen Patienten anonym erfolgte, war eine nachträgliche schriftliche Einwilligung nicht erforderlich.

\subsection{Patientenmanagement}

Alle Patienten wurden entweder in der Universitätsklinik der RWTH Aachen oder in der Universitätsmedizin Göttingen intensivmedizinisch versorgt und durch die jeweils ansässige neurochirurgische Abteilung behandelt. Sie wurden in beiden Kliniken nach gleichem Schema behandelt, da die Behandlungsstrategien durch Prof. Dr. V. Rohde von der Aachener zur Göttinger Klinik übertragen wurden (Prof. Rohde war zuvor Oberarzt der Aachener Klinik). Die prä- und postoperative Diagnostik umfasste neben cCT-Aufnahmen direkt nach Einlieferung, vier Stunden postoperativ und bei Bedarf zusätzlich im weiteren postoperativen Verlauf auch eine konsequente Vasospasmuskontrolle mittels täglicher transkranieller Dopplersonographie (TCD) innerhalb der ersten 14 Tage nach chirurgischem Eingriff. Eine eventuelle Vasospasmustherapie erfolgte durch modifizierte Triple$H$-Therapie (Hypertonie, moderate Hypervolämie, keine Hämodilution).

Am Ende des stationären Aufenthaltes erfolgte abschließend zum Zeitpunkt der Entlassung eine Beurteilung des klinischen Zustands nach erfolgter Therapie anhand der Glasgow Outcome Scale (GOS, Jennett und Bond 1975). 
Die gesammelten Daten der UMG wurden den bereits vorhandenen, nach gleichen Kriterien zusammengestellten Daten der Aachener Klinik hinzugefügt und in einer gemeinsamen Datenbank ausgewertet.

\subsubsection{Art der Intervention}

Die Behandlung der Subarachnoidalblutung erfolgte in beiden Kliniken grundsätzlich auf gleiche Art und Weise. Nach abgeschlossener Diagnostik wurde interdisziplinär entschieden, ob eine endovaskuläre oder eine mikrochirurgische Therapie durchgeführt wird. Da sich die Methode des Coilings im Laufe des Studienzeitraumes zunächst entwickelte und erst während der Göttinger Erfassungsperiode als standardisierte Behandlung etablierte, ist folglich das Patientenkollektiv der Coiling-Patienten in der Aachener Klinik sehr klein gewesen. Sowohl das Coiling als auch das Clipping der Aneurysmen erfolgte in beiden Kliniken nach dem gleichen Protokoll zur Behandlung von Patienten mit SAB.

\subsection{Ausgewertete bildgebende Verfahren}

Jeder Patient wurde nach Einlieferung und stationärer Aufnahme im Rahmen der initialen Standard-Diagnostik mit einer kranialen Computertomographie (cCT) untersucht. In den daraus hervorgehenden Schnittbildern des Schädels wurden das Ausmaß sowie die Lokalisation der Blutung festgestellt, wodurch sich in vielen Fällen bereits auch die Lage des ursächlichen Aneurysmas eingrenzen ließ. Des Weiteren ließ sich in der cCT-Aufnahme feststellen, ob es bei der Blutung zu einem Ventrikeleinbruch - einer Ausbreitung des Blutes in den von den vier Ventrikeln gebildeten inneren Liquorraum - gekommen ist. Auch eine sich von der subarachnoidalen Blutung ausweitende intrazerebrale Blutung (ICB) mit einhergehender Schädigung des Hirnparenchyms war im cCT erkennbar (s. Abbildung 2-1 D). Das Ausmaß der Blutung wurde nach der FisherKlassifikation (Grad I - IV) eingeteilt (Fisher et al. 1980, s. Tabelle 2-1 und Abbildung 2-1). 


\section{Grad cCT-Befund}

\begin{tabular}{c|l}
\hline I & kein Blut im Subarachnoidalraum sichtbar \\
II & $\begin{array}{l}\text { diffuse Blutansammlungen oder Blutgerinnsel mit Schichtdicke }<1 \mathrm{~mm} \\
\text { III }\end{array}$ \\
IV & $\begin{array}{l}\text { lokale Blutgerinnsel oder Blutansammlungen mit Schichtdicke }>1 \mathrm{~mm} \\
\text { intraventrikuläre oder intrazerebrale Blutung (mit oder ohne Blutansammlung im }\end{array}$ \\
\hline
\end{tabular}

Tabelle 2-1: SAB-Klassifikation nach Fisher
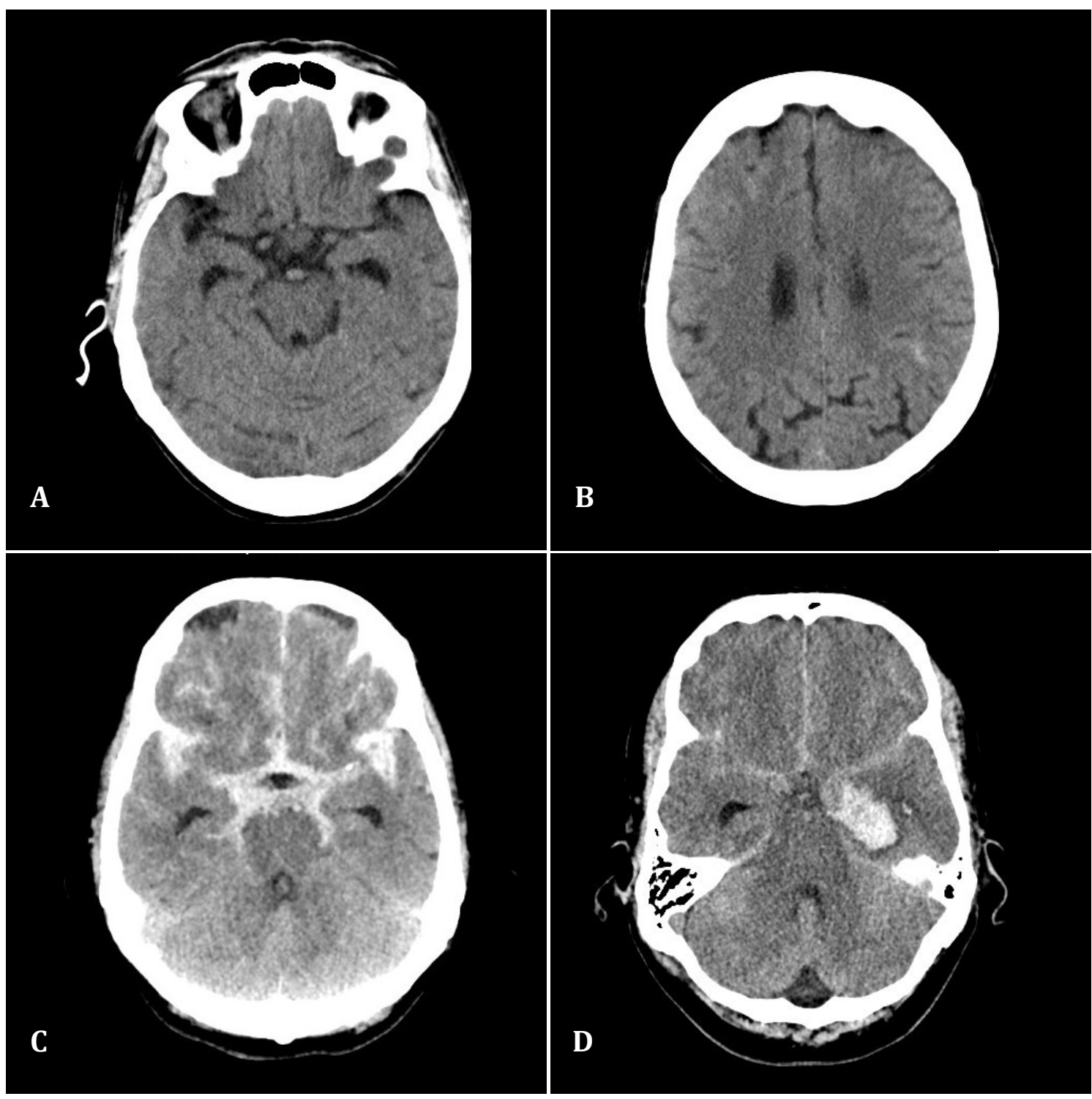

Abbildung 2-1: Axiale cCT-Aufnahmen mit Nachweis von aSABs verschiedener Fisher-Grade.

Fisher-Grade I [A], II [B], III [C] und IV (mit ICB; [D]). (Zur Verfügung gestellt vom Institut für Diagnostische und Interventionelle Neuroradiologie, UMG.) 
Zusätzlich zur präoperativen cCT-Untersuchung wurden bei allen Patienten auch angiographische Untersuchungen durchgeführt.

Zur genauen Lokalisation des Aneurysmas/der Aneurysmen wurden die Patienten ebenfalls mittels CT-Angiographie (CTA) und/oder digitaler Subtraktionsangiographie untersucht. Bei dem Verfahren der CTA wird ein radiologisch sichtbares Kontrastmittel in den zerebralen Blutkreislauf gegeben, das es ermöglicht, in der anschließenden CT-Aufnahme den exakten Verlauf der Blutgefäße darzustellen. Vorhandene Gefäß-Malformationen oder -Aussackungen können so genau erkannt und diagnostiziert werden. Anhand dieser Untersuchung wurden die Anzahl, die Lage und die zuführenden Gefäße der vorhandenen Aneurysmen festgestellt.

Der Goldstandard in der Detektion zerebraler Aneurysmen ist die digitale Subtraktionsangiographie (DSA). Auch hierbei erfolgt eine Kontrastmittelgabe, allerdings nicht, wie bei der konventionellen Angiographie, vor der Untersuchung, sondern erst im Laufe der Untersuchung. So entstehen in einer zeitlichen Abfolge Aufnahmen der zu betrachtenden Region, die die entsprechenden Blutgefäße sowohl kontrastmittelfrei als auch kontrastmittelgefüllt darstellen. Diese Bilder werden digital voneinander subtrahiert. Auf diese Weise ergibt sich eine Darstellung, die lediglich die Strukturen zeigt, die sich während der Kontrastmittelgabe bildgebend verändert haben: die Blutgefäße (Harrington et al. 1982). Alle anderen umgebenden Strukturen werden durch die Subtraktion herausgerechnet, sodass eine sehr exakte, alleinige Darstellung der Gefäße (und damit auch der Aneurysmen) erfolgen kann (s. Abbildung 2-2).

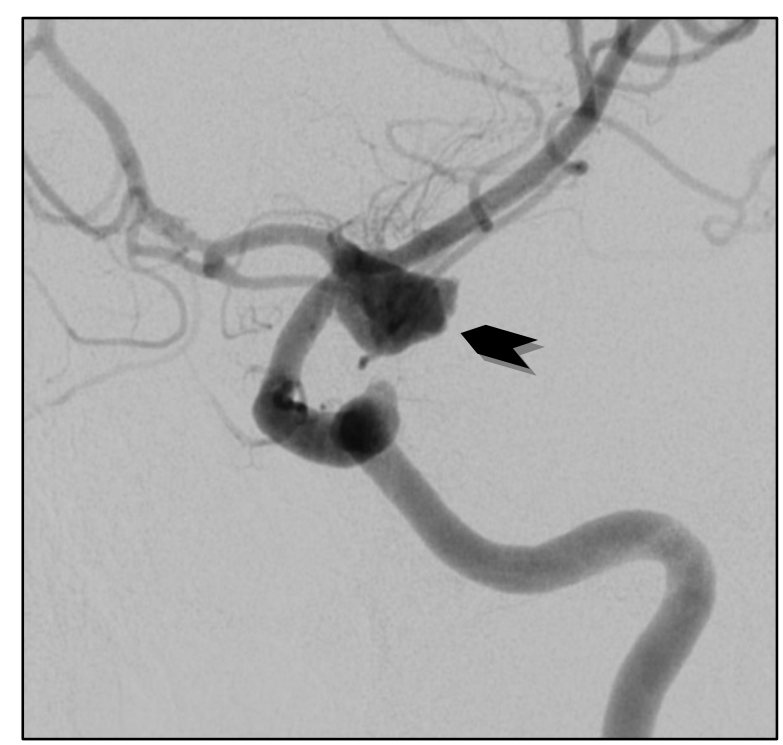

Abbildung 2-2: DSA-Aufnahme eines ACI-Aneurysmas.

(S. Pfeil; zur Verfügung gestellt vom Institut für Diagnostische und Interventionelle Neuroradiologie, UMG.) 
In manchen Fällen wurden als initiale Bildgebung Magnetresonanztomographien (MRT) angefertigt. Dies betraf vor allem Patienten, bei denen cCT-Untersuchungen keinen Blutungsnachweis ergaben (z.B. durch ein bereits einige Tage zurückliegendes Blutungsereignis) oder die Ursachen der Beschwerden nicht eindeutig und sicher zu diagnostizieren waren.

Anhand dieser bildgebenden Verfahren wurde für jeden Patienten das genaue Ausmaß der SAB, die Anzahl der vorhandenen bzw. rupturierten Aneurysmen und deren genaue Lage und Form an den Hirnarterien erkannt und festgehalten.

Die Aufnahme der Daten in die Datenbank und deren Auswertung erfolgte auf Basis der von den durchführenden Neuroradiologen erstellten Befundberichte.

\subsection{Datensammlung}

Für die in der Universitätsmedizin Göttingen behandelten Patienten stammten die studienrelevanten Daten aus den Patientenakten im Zentralarchiv der UMG. Sowohl aus den Übergabeprotokollen der einliefernden Notärzte als auch aus Aufnahme- und Behandlungsprotokollen der Stationen, die die Patienten nach Einlieferung versorgten, aus Anästhesie- und OP-Protokollen und aus Entlassungsbriefen der UMG und weiterbehandelnder Kliniken wurden notwendige Informationen für die Datensammlung entnommen. Zur Recherche lagen die Patientenakten der Neurochirurgie und der Anästhesiologie vor, zusätzlich wurden Daten aus den Dokumentationsprogrammen ixserv.4 (ixmid Software Technologie GmbH, Köln, DE) und ICCA (IntelliSpace Critical Care and Anesthesia; Philips, Amsterdam, NL) hinzugefügt. Die gesammelten Daten wurden mittels Microsoft Excel 2010 (Microsoft, Redmond, USA) in einer umfassenden Tabelle anonymisiert zusammengefügt. 


\subsubsection{Klinische Daten}

Für die Patienten mit eingetretener aSAB wurde der klinische Zustand bei der stationären Aufnahme nach der Glasgow Coma Scale (GCS; Teasdale und Jennett 1974, s. Tabelle 2-2) und die Schwere der SAB nach dem Hunt und Hess-Grad (H\&H; Hunt und Hess 1968, s. Tabelle 2-3) beurteilt.

\begin{tabular}{|c|c|c|}
\hline Kriterium & Antwort & Punkte \\
\hline \multirow[t]{4}{*}{ Augen öffnen } & Spontane Öffnung & 4 \\
\hline & Öffnung auf Ansprache & 3 \\
\hline & Öffnung auf Schmerzreiz & 2 \\
\hline & keine Reaktion & 1 \\
\hline \multirow[t]{5}{*}{ Verbale Kommunikation } & zu Zeit, Ort und Person orientiert, konversationsfähig & 5 \\
\hline & desorientiert, verwirrt, konversationsfähig & 4 \\
\hline & unzusammenhängende Antworten, erkennbare Wörter & 3 \\
\hline & unverständliche Laute & 2 \\
\hline & keine verbale Reaktion & 1 \\
\hline \multirow[t]{6}{*}{ Motorische Reaktion } & Reaktion bei Aufforderung & 6 \\
\hline & gezielte Bewegungen bei Schmerzreiz & 5 \\
\hline & ungezielte Bewegungen bei Schmerzreiz & 4 \\
\hline & abnormale Beugesynergismen bei Schmerzreiz, Dekortikationsstarre & 3 \\
\hline & abnormale Strecksynergismen bei Schmerzreiz, Dezerebrationsstarre & 2 \\
\hline & keine motorische Reaktion & 1 \\
\hline
\end{tabular}

Tabelle 2-2: Glasgow Coma Scale nach Teasdale und Jennett 


\begin{tabular}{c|l}
\hline Grad & Kriterien \\
\hline I & keine Symptome oder leichte Kopfschmerzen und/oder leichter Meningismus \\
Ia & isolierte Hirnnervenparese, kein Meningismus \\
II & $\begin{array}{l}\text { moderate bis schwere Kopfschmerzen, ausgeprägter Meningismus, keine neurologischen Defizite } \\
\text { außer Hirnnervenparesen }\end{array}$ \\
III & Schläfrigkeit, Verwirrtheit oder milde fokale Defizite \\
IV & $\begin{array}{l}\text { Stupor, moderate bis schwere Hemiparese, mögliche beginnende Dezerebrationsstarre und vegetative } \\
\text { Störungen }\end{array}$ \\
V & tiefes Koma, Dezerebrationsstarre, moribundes Erscheinungsbild \\
\hline
\end{tabular}

Tabelle 2-3: SAB-Klassifikation nach Hunt und Hess

Das Auftreten neurologischer Defizite (Hemiparese, Aphasie, Vigilanzminderung) sowie epileptischer Anfälle wurde zusätzlich zu den biometrischen Daten (Alter, Geschlecht) und Vitalparametern (Puls, Blutdruck und Körpertemperatur bei Einlieferung) aufgenommen.

Ein eventuell im Vorfeld aufgetretenes „Warn-Ereignis“ im Sinne von milder auftretenden Symptomen, ähnlich denen der SAB (sog. minor leak), wurde ebenfalls dokumentiert. In einigen Fällen kommt es wenige Tage bis Wochen vor der aSAB zu einem milderen Vorereignis, bei dem bereits eine kleine Blutung aus dem Aneurysma stattfindet und darauf schließen lässt, dass es im weiteren Verlauf zu einer Ruptur des Aneurysmas kommen kann (Leblanc 1987).

Nach Auswertung der radiologischen Befunde und Klassifizierung der SAB nach Fisher wurde das Vorhandensein einer Ventrikelblutung und/oder einer ICB erfasst. Die Anzahl der Aneurysmen und deren genaue Lage fanden ebenfalls Eingang in die Datensammlung der präoperativen Diagnostik.

\subsubsection{Therapeutische Maßnahmen}

Für das therapeutische Vorgehen wurde als erster Parameter vermerkt, ob präoperativ eine Liquordrainage zur Behandlung eines akuten Hydrozephalus (Wasserkopf) angelegt wurde oder nicht. Hierbei wurde zwischen lumbaler 
Liquordrainage (LD) und externer Ventrikeldrainage (EVD) unterschieden. Lumbaldrainagen erhielten Patienten mit gut eingestuftem Hunt und Hess-Grad (I - III), bei schlechtem Zustand (H\&H-Grad IV und V) wurde eine EVD zur Senkung des Hirndrucks präoperativ angelegt. Falls notwendig wurde bei Insuffizienz der liegenden Drainage (LD oder EVD) zusätzlich eine Drainage der jeweils anderen Art gelegt. Alle Patienten, die einer nicht-chirurgischen Therapie zum Aneurysmaverschluss unterzogen wurden oder gänzlich ohne Intervention blieben, sind im nächsten Schritt aus der Datenbank ausgeschlossen worden, sodass lediglich die Patienten evaluiert wurden, die ein Clipping der Aneurysmen erfahren haben. Bei diesen Patienten wurde zusätzlich festgehalten, in welchem zeitlichen Abstand zum Eintreten der SAB die Operation stattfand. Als abschließender Faktor zur Intervention wurde dokumentiert, ob während der Operation ein temporäres Clipping stattgefunden hat. Die Auswertung des Operationsverlaufes erfolgte über das Auftreten OP-bedingter Defizite (Paresen, Aphasie) und die Beurteilung postoperativer cCT-Aufnahmen, die 4 Stunden nach Ende der Operation durchgeführt und in Bezug auf das Neuauftreten von Infarkten im Vergleich zu den präoperativen Aufnahmen evaluiert wurden. Postoperativ wurden alle Patienten für einen Zeitraum von mindestens 14 Tagen intensivmedizinisch überwacht.

\subsubsection{Vasospasmus}

Postoperativ erfolgte bei allen Patienten eine enge Vasospasmuskontrolle. Während der ersten 14 Tage nach der Operation wurde täglich mittels transkranieller Dopplersonographie (TCD) die Flussgeschwindigkeit der hirnversorgenden Arterien untersucht. Für die Doppleruntersuchung wurde der transtemporale Zugang genutzt, um die mittleren zerebralen Flussgeschwindigkeiten zu ermitteln. Hier findet auch heute noch die von Aaslid et al. beschriebene Vorgehensweise Anwendung, nach der die dünne Knochenschicht der Temporalregion genutzt wird, um niedrigfrequente Ultraschallwellen ins Schädelinnere zu senden (Aaslid et al. 1982). Die Aufzeichnung erfolgte in Zentimetern pro Sekunde $(\mathrm{cm} / \mathrm{s})$ durch einen $2 \mathrm{MHz}$ getakteten Doppler-Ultraschallkopf. 
Die Grenze zur Definition eines vorliegenden Vasospasmus lag in diesem Fall bei der Überschreitung einer mittleren Flussgeschwindigkeit von $120 \mathrm{~cm} / \mathrm{s}$ (Aaslid et al. 1984) oder einem sprunghaften Anstieg im Vergleich zum Vortags-Wert um mehr als $50 \mathrm{~cm} / \mathrm{s}$. Zur weiteren Diagnostik wurden teilweise ebenfalls erneute Angiographien durchgeführt. In den letzten ca. 2 Jahren des beobachteten Studienzeitraums standen ebenfalls Perfusions-cCT-Untersuchungen für die Diagnostik zur Verfügung.

Darüber hinaus fand eine Dokumentation der Vasospasmustherapie statt, die in der Regel eine standardmäßige Gabe von Nimodipin (10 ml/h) für 14 Tage beinhaltete. Bei behandlungsbedürftigen Vasospasmen wurde eine sog. Triple-H-Therapie (Hypertension, Hypervolämie, Hämodilution) in modifizierter Form eingesetzt. Eine Hämodilution wurde hierbei nicht durchgeführt. Die Hypervolämie erfolgte nur in normalem bis mildem Ausmaß (Zentralvenendruck zwischen 8 und $12 \mathrm{mmHg}$ ). Geachtet wurde auf eine strenge Hypertension mit systolischem Blutdruck zwischen 160 und 180 mmHg. Während der gesamten Studiendauer (1990 bis 2013) wurde die Vasospasmustherapie nach diesen Vorgaben standardmäßig durchgeführt. Interventionelle Therapiemaßnahmen wie Ballondilatation oder intraarterielle Nimodipin-Gabe wurden in Einzelfällen nach erneuter Angiographie und gezielter interdisziplinärer Absprache durchgeführt und gesondert festgehalten.

\subsubsection{Postoperative Maßnahmen}

Im weiteren postoperativen Verlauf wurde auf das Auftreten verspäteter neurologischer Defizite geachtet (DIND - Delayed Ischaemic Neurological Deficits, wie z.B. Paresen, Aphasie, Verhaltensauffälligkeiten, Bewusstseinstrübung). Mittels cCT-Aufnahmen erfolgte dazu eine Diagnostik eventuell vorliegender - auch spasmusbedingter - Infarkte. Bei entsprechender Indikation wurden - aufgrund moderner Entwicklung nur in den letzten Jahren der Studie - neben erneuten Angiographien routinemäßig auch zusätzliche Untersuchungsmöglichkeiten wie Perfusions-cCTs der von Vasospasmen betroffenen Gebiete durchgeführt. In einigen Fällen erfolgte eine intraarterielle Intervention zur Behandlung der Vasospasmen. Ballondilatationen der betroffenen Gefäße wurden ebenso 
durchgeführt wie die direkte intraarterielle Gabe von Nimodipin zur Relaxation der spastischen Gefäße.

Bei chronischem malresorptivem Hydrozephalus erfolgte im postoperativen Verlauf die Einrichtung einer permanenten Liquorableitung durch Anlage eines ventrikulo-peritonealen Shunts (VP-Shunts) zum Ablauf des überschüssigen Liquors und zur Senkung des Hirndrucks.

\subsubsection{Outcome}

Zur abschließenden Beurteilung des Patientenzustands wurde für jeden Patienten zum Zeitpunkt der Entlassung eine Einstufung nach der Glasgow Outcome Scale (GOS; Jennett und Bond 1975, s. Tabelle 2-4) vorgenommen. Nach gleichen Kriterien erfolgten ebenfalls Kontrolluntersuchungen, die den Verlauf des Patientenzustandes in den Monaten nach der Erkrankung dokumentierten.

\section{Grad Kriterien}

\begin{tabular}{c|l}
\hline I & Tod \\
II & $\begin{array}{l}\text { vegetativer Zustand; schwerste neurologische Schädigungen mit anhaltendem Zustand der } \\
\text { Reaktionslosigkeit und Abhandensein von höheren Geistesfunktionen }\end{array}$ \\
III & $\begin{array}{l}\text { schwere Behinderung; schwere neurologische Schädigungen, dauerhafte Pflegebedürftigkeit aufgrund } \\
\text { von mentaler oder physischer Behinderung }\end{array}$ \\
IV & $\begin{array}{l}\text { mäßige Behinderung; leichte neurologische Defizite, keine dauerhafte Pflegebedürftigkeit in Belangen } \\
\text { des alltäglichen Lebens }\end{array}$ \\
V & $\begin{array}{l}\text { leichte bis keine Behinderung; eventuell leichte neurologische Defizite ohne Beeinträchtigung des } \\
\text { alltäglichen Lebens }\end{array}$ \\
\hline
\end{tabular}

Tabelle 2-4: Glasgow Outcome Scale nach Jennett und Bond 


\subsection{Software}

Citavi 5

ICCA (IntelliSpace Critical Care

and Anesthesia)

ixserv.4

Microsoft Excel 2010

Microsoft Word 2010

SAS version 9.2

STATISTICA 12
Swiss Academic Software GmbH (Wädenswil, CH)

Philips (Amsterdam, NL)

ixmid Software Technologie GmbH (Köln, DE)

Microsoft (Redmond, WA, USA)

Microsoft (Redmond, WA, USA)

SAS Institute Inc. (Cary, USA

StatSoft, Inc. (Tulsa, OK, USA)

\subsection{Statistik}

Die statistischen Auswertungen und grafischen Darstellungen wurden mit Hilfe der Softwares STATISTICA 12, SAS version 9.2 und Microsoft Excel 2010 erstellt. Metrische Studiendaten wurden per deskriptiver Statistik ausgewertet und als Mittelwerte \pm Standardabweichung dargestellt. Für kategorielle Daten wurden absolute und relative Häufigkeiten ermittelt. Häufigkeitsverteilungen ermöglichten die Auswertung der einzelnen aufgenommenen Variablen der Datenbank. Einfache Vergleichsstatistiken dieser Häufigkeitsverteilungen wurden als Kreuztabellen erstellt und mittels Chi-Quadrat-Test auf Signifikanz untersucht. Um den Einfluss des temporären Clippings und verschiedener weiterer Variablen auf den postoperativen Vasospasmus $\mathrm{zu}$ berechnen, wurde eine multivariate Analyse anhand einer multiplen logistischen Regressionsanalyse durchgeführt. Hierzu wurde ein binäres Logit-Modell erstellt, welches den wahrscheinlichen Effekt einzelner ausgewählter Variablen auf das Vorhandensein eines Vasospasmus analysierte. Per Maximum-Likelihood-Schätzungen und Odds Ratio mit entsprechenden $95 \%$ Konfidenz-Intervallen wurde der Effekt der einzelnen untersuchten Parameter auf den Vasospasmus beschrieben. 
In allen Berechnungen wurde eine statistische Signifikanz angenommen, wenn sich durch die Analyse zeigte, dass die untersuchten Gruppen eine Irrtumswahrscheinlichkeit von $\leq 0,05$ auswiesen ( $p \leq 0,05)$. 


\section{Ergebnisse}

\subsection{Patientenkollektiv}

Aus dem Zeitraum zwischen 1990 und 2005 in der Universitätsklinik Aachen fanden insgesamt 456 Patienten, die unter einer aneurysmatischen Subarachnoidalblutung litten, Eingang in die Datenbank. Hinzu kamen aus der Zeit zwischen 2005 und 2013299 Patienten, die mit demselben Krankheitsbild in der Universitätsklinik Göttingen behandelt wurden. Bei diesen insgesamt 755 Patienten wurden 993 Aneurysmen diagnostiziert, die in der Folge entweder chirurgisch (Clipping), endovaskulär (Coiling) oder ohne weitere Intervention therapiert wurden. Zu 3 der 755 Patienten standen keine Daten zur Therapie zur Verfügung, von den restlichen 752 blieben 30 ohne Intervention (4,0\%), 187 wurden per Coiling therapiert $(24,9 \%)$ und 535 erfuhren einen operativen Aneurysmaverschluss durch Clipping (71,1\%).

In der Aachener Klinik erfolgte bei 398 Patienten eine chirurgische Therapie $(87,9 \%, n=456)$. Die Göttinger Klinik führte bei 137 Patienten einen Aneurysma-Verschluss durch Clipping durch $(45,8 \%, \mathrm{n}=299)$.

In der Folge wurden für diese Arbeit

\begin{tabular}{|c|c|c|}
\hline & Anzahl (n) & $\%$ \\
\hline Gesamt & 535 & \\
\hline \multicolumn{3}{|l|}{ Geschlecht } \\
\hline männlich & 184 & 34,4 \\
\hline weiblich & 351 & 65,6 \\
\hline Alter (in Jahren) & $48,4( \pm 12,54)$ & \\
\hline$\leq 60$ Jahre & 468 & 87,5 \\
\hline$>60$ Jahre & 67 & 12,5 \\
\hline \multicolumn{3}{|l|}{ GCS } \\
\hline$<7$ & 136 & 26,7 \\
\hline $7-12$ & 56 & 11,0 \\
\hline$<12$ & 318 & 62,3 \\
\hline fehlend & 25 & \\
\hline
\end{tabular}

\begin{tabular}{lcc} 
H\&H-Grad & & \\
I & 93 & 17,4 \\
II & 139 & 26,0 \\
III & 141 & 26,3 \\
IV & 56 & 10,5 \\
V & 106 & 19,8 \\
\hline Fisher-Grad & & \\
I & 4 & 0,8 \\
II & 73 & 13,7 \\
III & 174 & 32,7 \\
IV & 281 & 52,8 \\
fehlend & 3 & \\
\hline
\end{tabular}

\begin{tabular}{lcc} 
GOS & & \\
1 & 60 & 16,7 \\
2 & 6 & 1,7 \\
3 & 47 & 13,1 \\
4 & 46 & 12,8 \\
5 & 200 & 55,7 \\
fehlend & 4 & \\
\hline
\end{tabular}

Tabelle 3-1: Patientendaten GCS: Glasgow Coma Scale; H\&H: Hunt und Hess; GOS: Glasgow Outcome Scale lediglich die 535 Patienten beachtet, bei denen eine chirurgische Therapie durchgeführt wurde (s. Tabelle 3-1). 
Somit ergab sich ein zu analysierendes Gesamtkollektiv von 535 Patienten mit 695 Aneurysmen, die im Zeitraum von 1990 bis 2013 in Aachen und Göttingen nach eingetretener aneurysmatischer SAB mit Clipping versorgt wurden.

\subsection{Klinische Daten und Befunde}

\subsubsection{Biometrische Daten}

Unter den 535 Patienten mit Aneurysma-Clipping befanden sich 351 weibliche (65,6 \%) und 184 männliche (34,4 \%) Patienten. Das durchschnittliche Alter lag bei 48,4 \pm 12,4 (Standardabweichung) Jahren (s. Abbildung 3-1). Der jüngste Patient war zum Zeitpunkt des Blutungseintritts 18, der älteste 87 Jahre alt. Eine Dichotomisierung des Patientenkollektivs nach Alter ergab, dass 468 Patienten $(87,5 \%) 60$ Jahre oder jünger und 67 Patienten (12,5 \%) älter als 60 Jahre alt waren.

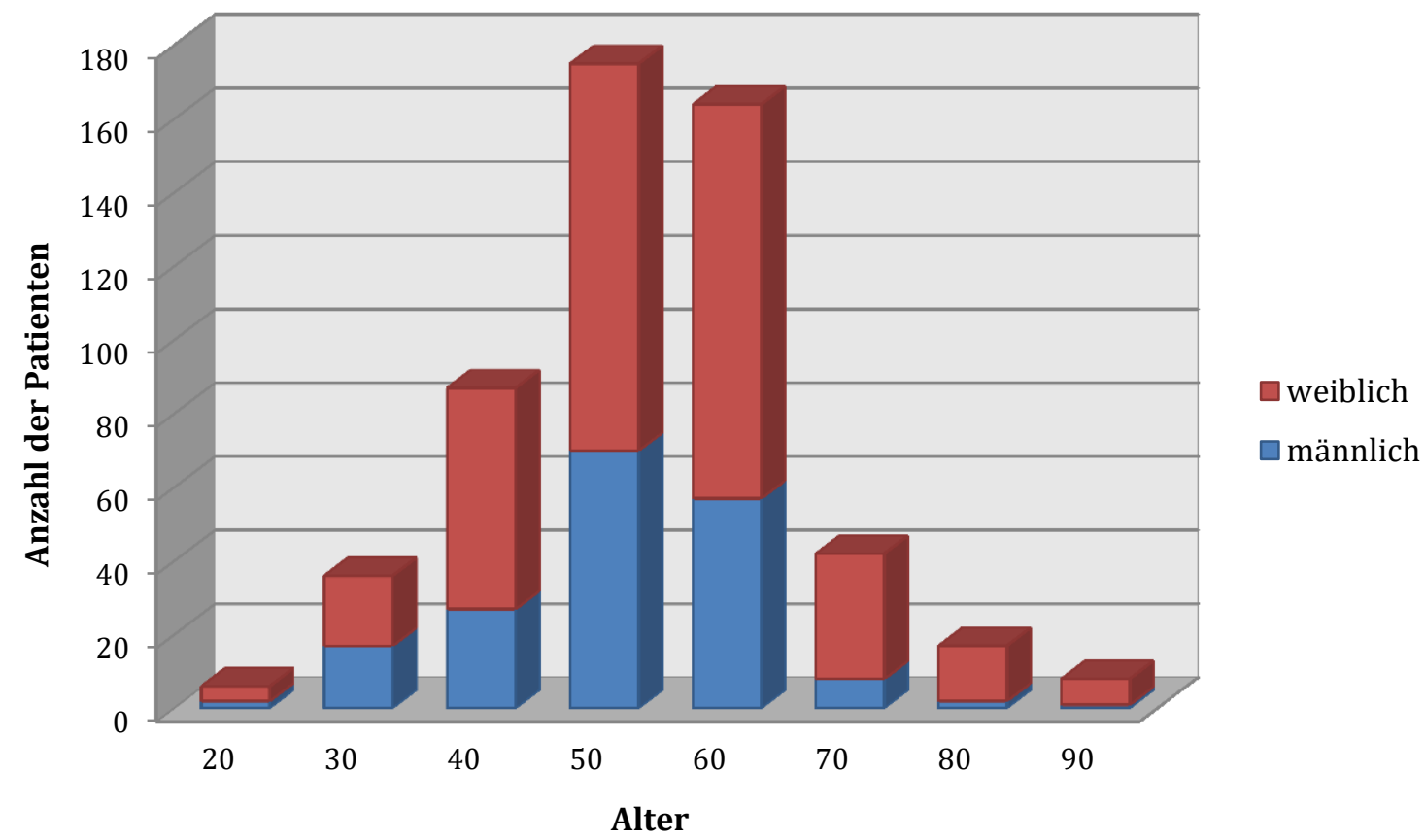

Abbildung 3-1: Altersverteilung des Patientenkollektivs 


\subsubsection{Aufnahmebefunde}

Der Zustand der Patienten bei stationärer Aufnahme wurde durch den Hunt und Hess-Grad (H\&H, s. Abbildung 3-2) und die Glasgow Coma Scale (GCS, s. Abbildung 3-3) erhoben.

Mit H\&H-Grad I präsentierten sich 93 Patienten (17,4 \%), 139 mit Grad II (26,0 \%), 141 und 56 mit Grad III und IV (26,3 \% und 10,5 \%) und 106 mit dem höchsten Grad V (19,8 \%). Die dichotomisierte Einteilung in milde (H\&H-Grad I - III) und schwere SAB (H\&H-Grad IV und V) ergibt, dass beinahe ein Drittel aller untersuchten Patienten an einer als schwer eingestuften SAB litt (30,3\% mit H\&H-Grad IV - V, 69,7 \% mit H\&H-Grad I-III).

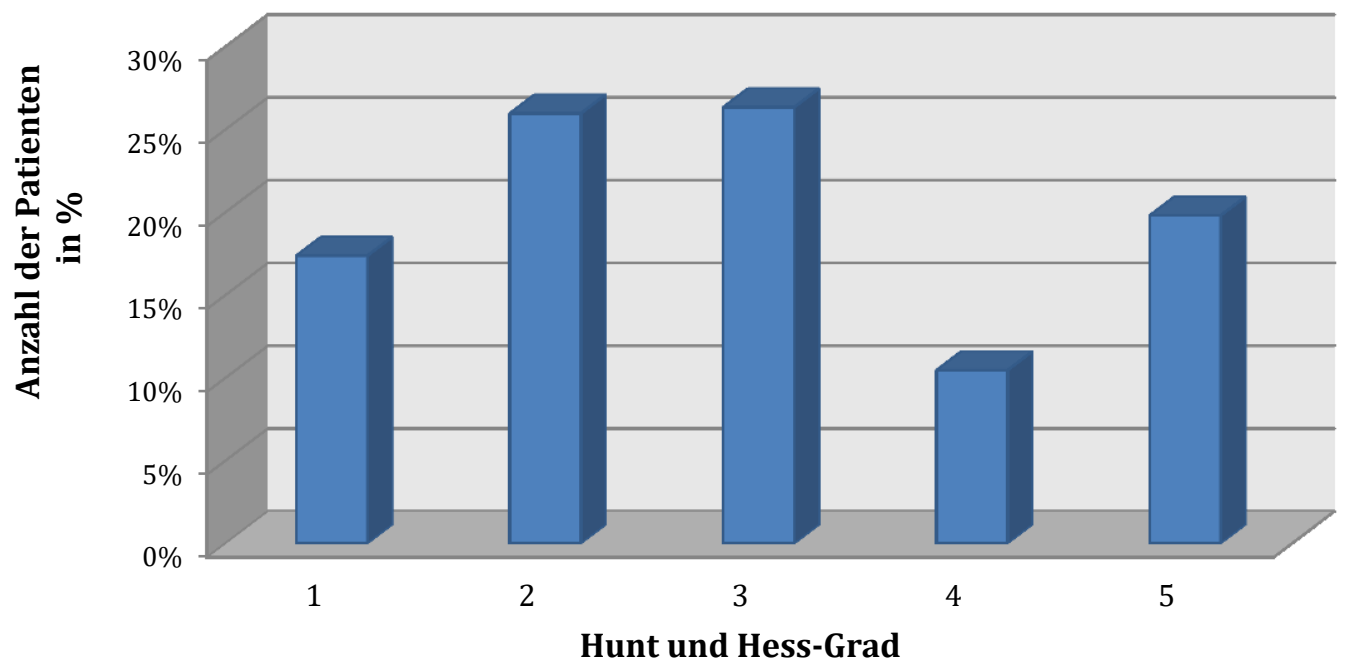

Abbildung 3-2: Patientenverteilung nach Hunt und Hess

Die Glasgow Coma Scale wurde zur besseren Übersichtlichkeit in drei Gruppen unterteilt ( $\mathrm{n}=510)$ : Schlechter Zustand mit schweren Hirnschädigungen und starken Einschränkungen (Gruppe I, GCS < 7), moderate Hirnschädigungen und eingeschränkter Zustand (Gruppe II, GCS 7-12) sowie guter Zustand mit leichten Einschränkungen (Gruppe III, GCS $\geq 13$ ). Die Mehrzahl der Patienten ( $n=318$ ) fiel bei der Aufnahme in die dritte Gruppe (62,3 \%), der nächsthöhere Anteil ( $n=136)$ kam in klinisch schlechtem Zustand (Gruppe I) zur Einlieferung (26,7\%). Die zweite Gruppe enthielt 56 Patienten (11,0 \%). Eine genaue Darstellung der Patientenverteilung zeigt Abbildung 3-3. 


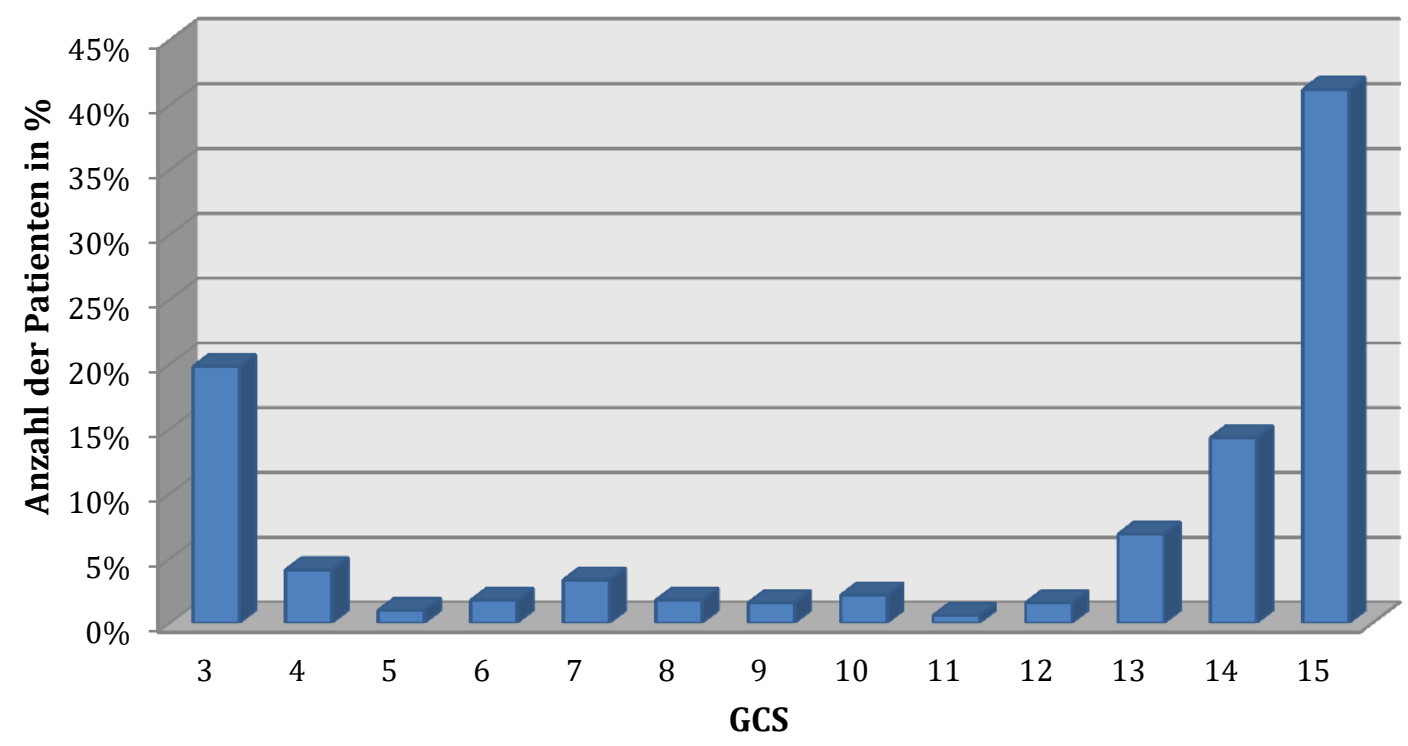

Abbildung 3-3: Patientenverteilung nach Glasgow Coma Scale

Bei 13,7 \% der Patienten ( $\mathrm{n}=506)$ konnte im Vorfeld der SAB eine Warnblutung (minor leak) diagnostiziert werden. Vorhandene neurologische Defizite wurden wie folgt festgestellt: Bei 68 Patienten manifestierte sich eine Hemiparese (13,8\%, $\mathrm{n}=493$ ). Epileptische Anfälle in Zusammenhang mit der SAB traten bei 50 Patienten auf $(9,8 \%, \mathrm{n}=512)$ und unter Anisokorie litten 72 Patienten $(14,1 \%$, $\mathrm{n}=511)$.

Zusätzlich wurden die Vitalparameter Blutdruck (systolisch, diastolisch), Puls und Körpertemperatur erfasst. Durchschnittlich ergaben sich Werte von 144,27 mmHg $( \pm 29,23)$ systolischen Drucks und $88,01 \mathrm{mmHg}( \pm 18,12)$ diastolischen Drucks bei Einlieferung in das Klinikum, bei einem Puls von 80,09 Schlägen/min $( \pm 17,89)$ und einer Körpertemperatur von $36,81^{\circ} \mathrm{C}( \pm 3,63)$.

\subsubsection{Voruntersuchungen}

Nach Auswertung der durchgeführten cCT-, CTA-, DSA- und MRT-Untersuchungen der Patienten ergab sich bei den 535 Patienten eine Anzahl von insgesamt 695 diagnostizierten Aneurysmen (s. Tabelle 3-2). Bei 111 Patienten wurden multiple Aneurysmen festgestellt (20,8\%). Anhand der durchgeführten Diagnostik ließen sich die Aneurysmen wie folgt lokalisieren: A. cerebri media (MCA): 239 (34,4 \%), A. communicans anterior (AComA): 191 (27,5\%), A. communicans posterior (PComA): 85 (12,2 \%), A. carotis interna (ACI): $61 \quad(8,8 \quad \%)$, 
A. cerebri anterior (ACA): $26 \quad(3,7 \%)$,

A. cerebelli inferior posterior (PICA):

22 (3,2 \%), A. basilaris (BA): 15

$(2,2 \%), A$. choroidea anterior (AChoA): 14 (2,0 \%), A. pericallosa:

13 (1,9\%), A. ophtalmica (OA): 11 (1,6\%), A. vertebralis (VA): 7 (1,0\%),

A. cerebri posterior (PCA): 4 (0,6\%),

A. cerebelli superior (SUCA): 4 $(0,6 \%)$, A. cerebelli inferior anterior (AICA): $2 \quad(0,3 \quad \%) . \quad \mathrm{Zu}$ einem Aneurysma war keine Lokalisationsangabe vorhanden $(0,1 \%)$.

Die Schwere und Ausbreitung der

\begin{tabular}{|c|c|c|}
\hline & Anzahl (n) & $\%$ \\
\hline Aneurysmen (gesamt) & 695 & \\
\hline multiple & 111 & 20,8 \\
\hline Lokalisation & 694 & \\
\hline anteriore Zirkulation & 602 & 86,8 \\
\hline posteriore Zirkulation & 92 & 13,2 \\
\hline fehlend & 1 & \\
\hline Fisher-Grad & 532 & \\
\hline I - II & 77 & 14,5 \\
\hline III - IV & 455 & 85,5 \\
\hline fehlend & 3 & \\
\hline
\end{tabular}

Tabelle 3-2: Aneurysma-Daten

SAB wurde nach der Fisher-Klassifikation eingeteilt (Verteilung s. Abbildung 3-4). Lediglich 4 Patienten wurden bei Fisher-Grad I eingestuft $(0,8 \%)$ und 73 bei Grad II (13,7 \%). Die deutliche Mehrheit der Patienten litt unter einer schweren SAB der Fisher-Grade III (174 Patienten, 32,7 \%) und IV (281 Patienten, 52,8 \%, n = 532). Bei 158 Patienten wurde eine intraventrikuläre Blutung $(36,4 \%, n=434)$ festgestellt und 137 erlitten eine intrazerebrale Blutung $(43,91 \%, n=312)$. Bei 72 Patienten wurde sowohl eine intraventrikuläre als auch eine intrazerebrale Blutung diagnostiziert $(26,0 \%, \mathrm{n}=277)$.

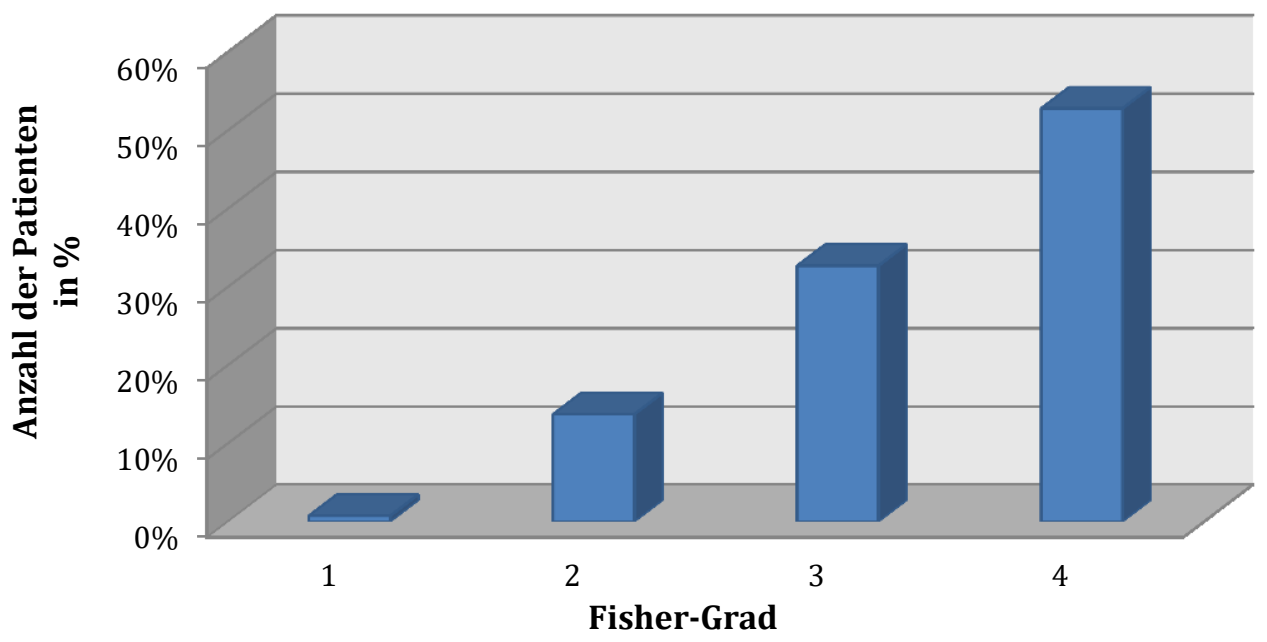

Abbildung 3-4: Patientenverteilung nach Fisher 


\subsubsection{Chirurgische Intervention}

Zu 481 der 535 operierten Patienten lagen Daten zu gelegten Ventrikeldrainagen vor, insgesamt erhielten 403 Patienten eine oder mehrere Drainagen (83,8 \%). Externe Ventrikeldrainagen (EVD) bekamen 176 Patienten (36,6 \%), lumbale Drainagen (LD) wurden bei 299 Patienten (62,2 \%) gelegt. Darunter befanden sich 72 Patienten (15,0 \%), bei denen sowohl eine EVD als auch eine LD gelegt wurden. Für die vorliegende Untersuchung wurden alle Patienten ausgeschlossen, die nicht einer chirurgischen Aneurysma-Therapie unterzogen wurden. Somit erfolgte bei allen für diese Studie betrachteten 535 Patienten ein Clipping des rupturierten Aneurysmas (100 \%, $\mathrm{n}=535)$. Von diesen 535 Patienten wurden 81,3\% innerhalb der ersten 72 Stunden nach Eintritt der Blutung operiert, 10,8 \% nach 4 bis 10 Tagen und die weiteren 7,9 \% zu einem späteren Zeitpunkt.

Während der Operation fand bei 180 Patienten ein temporäres Clipping statt $(n=455,39,6 \%)$.

\subsubsection{Postoperative Nachsorge}

Postoperativ wurden bei 103 Patienten (20,6 \%, n = 500) neurologische Defizite festgestellt. Diese umfassten sowohl bereits präoperativ vorliegende als auch durch die Operation bedingte Defizite. Das Auftreten von DIND wurde gesondert registriert und ausgewertet (s. Kapitel 3.2.6).

In Fällen eines anhaltenden Hydrozephalus wurde als langfristige Therapie ein ventrikulo-peritonealer Shunt angelegt. Dies wurde bei 97 Patienten (18,4\%, $\mathrm{n}=528$ ) durchgeführt.

Zur abschließenden Beurteilung des Erholungsgrades der Patienten nach Abschluss der Therapie wurde die Glasgow Outcome Scale (GOS) verwendet (Verteilung s. Abbildung 3-5). Ein Großteil der Patienten wies bei Entlassung einen guten bis sehr guten Zustand auf (61,0 \% mit GOS IV und V). Eine sehr gute Erholung zeigten 222 Patienten (GOS V, 41,8 \%), 102 Patienten wurden mit nur leichten bis mäßigen Einschränkungen entlassen (GOS IV, 19,2 \%). In deutlich schlechterem Zustand und mit schweren Defiziten und Einschränkungen verließen 124 Patienten die Klinik (GOS III, 23,4 \%). 4,7 \% der Patienten verblieben in dauerhaft vegetativem Zustand (GOS II) und 58 Patienten (10,9\%) überlebten die 
Erkrankung nicht (GOS I). Von vier Patienten lagen keine Daten zum Zustand bei Entlassung vor $(\mathrm{n}=531)$.

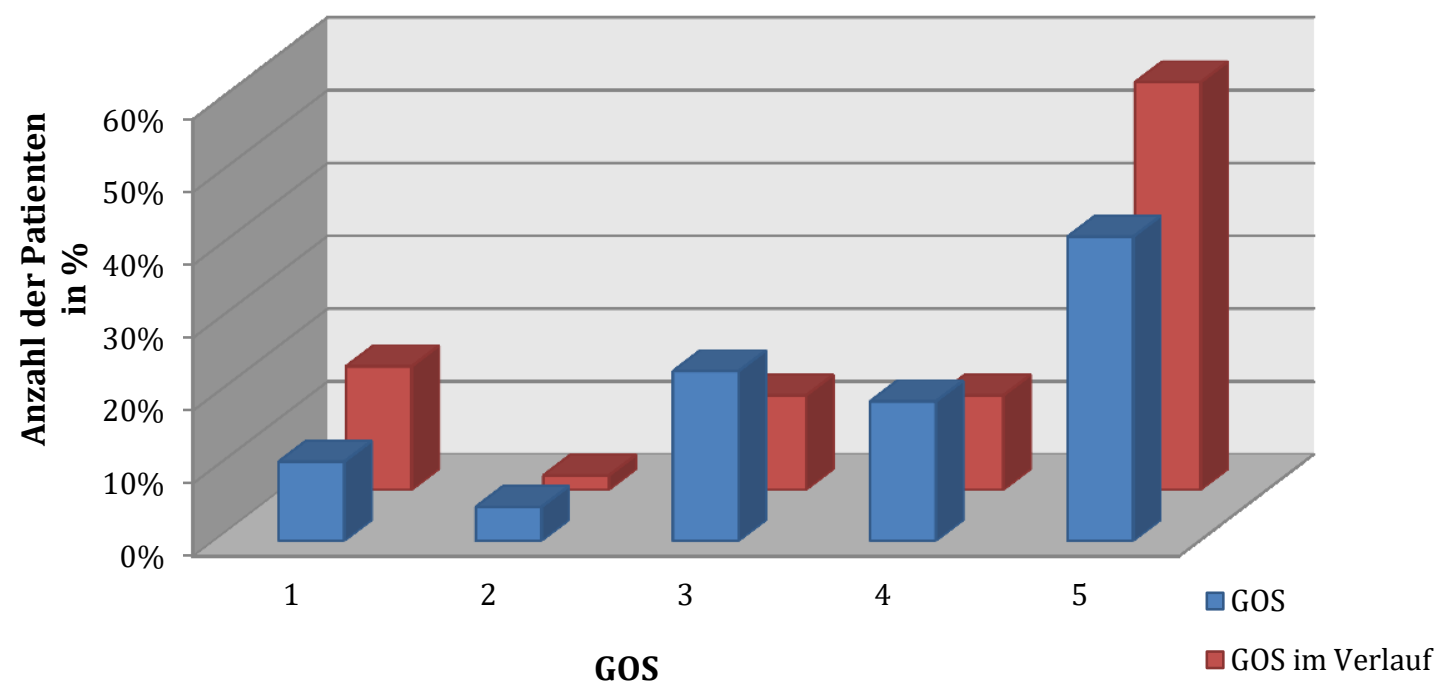

Abbildung 3-5: Patientenverteilung nach Glasgow Outcome Scale.

Zustand zum Zeitpunkt der Entlassung und im weiteren Verlauf nach mind. 6 Monaten.

Für eine langfristige Nachuntersuchung (mindestens 6 Monate nach Entlassung) standen Daten von 359 Patienten zur Verfügung (67,1\%). Hierbei ergab sich folgende Verteilung: GOS I (16,7 \%, 60 Patienten), GOS II (1,7 \%, 6 Patienten), GOS III (13,1 \%, 47 Patienten), GOS IV (12,8 \%, 46 Patienten) und GOS V (55,7 \%, 200 Patienten).

\subsubsection{Vasospasmus und DIND}

Es erfolgte für die ersten 14 Tage nach dem operativen Aneurysma-Verschluss eine tägliche Vasospasmuskontrolle mittels TCD. Im Verlauf dieser Zeit entwickelten 61,4\% der Patienten einen Vasospasmus ( $\mathrm{n}=523)$. Von den betroffenen Patienten wurden 96,7 \% bezüglich der Vasospasmen therapiert, wobei die konservative Therapie in der standardmäßigen prophylaktischen Gabe von Nimodipin bestand, gegebenenfalls unterstützt durch eine zusätzliche modifizierte Triple- $H$-Therapie. 103 Patienten $(22,5 \%, \mathrm{n}=457)$ entwickelten im Verlauf neurologische Defizite (DINDs) und bei 82 wurden Spasmus-induzierte Infarkte per cCT diagnostiziert $(23,4 \%, \mathrm{n}=351)$. 
Der Einfluss einzelner Variablen auf das Vorliegen eines Vasospasmus wurde initial durch Aufstellung von Kreuztabellen und anschließend mittels Chi-QuadratTest ermittelt. Hierbei zeigte sich, dass der initiale Einlieferungszustand eine signifikante Auswirkung auf das Auftreten postoperativer Vasospasmen hat $(p=0,04)$. Patienten in schlechtem Zustand entwickelten häufiger Vasospasmen als Patienten, die in gutem Zustand eingeliefert wurden. Die hierzu herangezogene GCS wurde für diese Analyse in Anlehnung an den WFNS-Grad (World Federation of Neurosurgical Societies SAH Grading Scale, Teasdale et al. 1988) dichotomisiert in (I): GCS 3 - 12 (schlechter bis moderater Zustand, WFNS-Grad IV - V) und (II): GCS 13 - 15 (guter Zustand, WFNS-Grad I - III). Ebenfalls signifikant wirkte sich die Ausdehnung der SAB, beurteilt nach Fisher, aus $(p<0,01)$. Auch hier fand eine Dichotomisierung des Fisher-Grads in leichte (Grad I und II) und schwere SAB (Grad III und IV) statt. Das Geschlecht der Patienten ( $p=0,07$ ) und die Schwere der SAB nach Hunt und Hess $(p=0,08)$ wiesen keinen signifikanten Einfluss auf den Vasospasmus auf. Hingegen hatte das Alter der Patienten eine deutliche Signifikanz. So zeigte sich, dass Patienten mit einem Alter $\leq 60$ Jahren deutlich häufiger Vasospasmen erleiden (65,2 \%) als ältere Patienten (> 60 Jahre, 31,7 \%, p $<0,001)$.

\subsubsection{Temporäres Clipping}

Sowohl in der Aachener als auch in der Göttinger Klinik fand während des gesamten Zeitraums der Untersuchung das temporäre intraoperative GefäßClipping Anwendung. Hierbei zeigt sich, dass die Aachener Klinik im Vergleich zur UMG generell einen deutlich höheren Anteil an operierten Patienten gegenüber endovaskulär behandelten Patienten hatte (87,3 \% vs. 45,8 \%). Ähnliches zeigte sich auch bei der Durchführung des temporären Clippings. Während in Aachen 161 Patienten einen temporären Gefäßverschluss erhielten (40,5 \%, n = 398), lag die Quote in Göttingen deutlich niedriger: Lediglich bei 19 Patienten wurde temporäres Clipping eingesetzt $(13,9 \%, \mathrm{n}=137)$. Insgesamt 180 Patienten $(39,6 \%, \mathrm{n}=455)$ erhielten intraoperativ eine zwischenzeitliche Gefäßausschaltung. 
Mittels Kreuztabellen und Chi-Quadrat-Test wurde initial der Einfluss des temporären Clippings untersucht. Hierbei zeigte sich, dass das temporäre Clipping

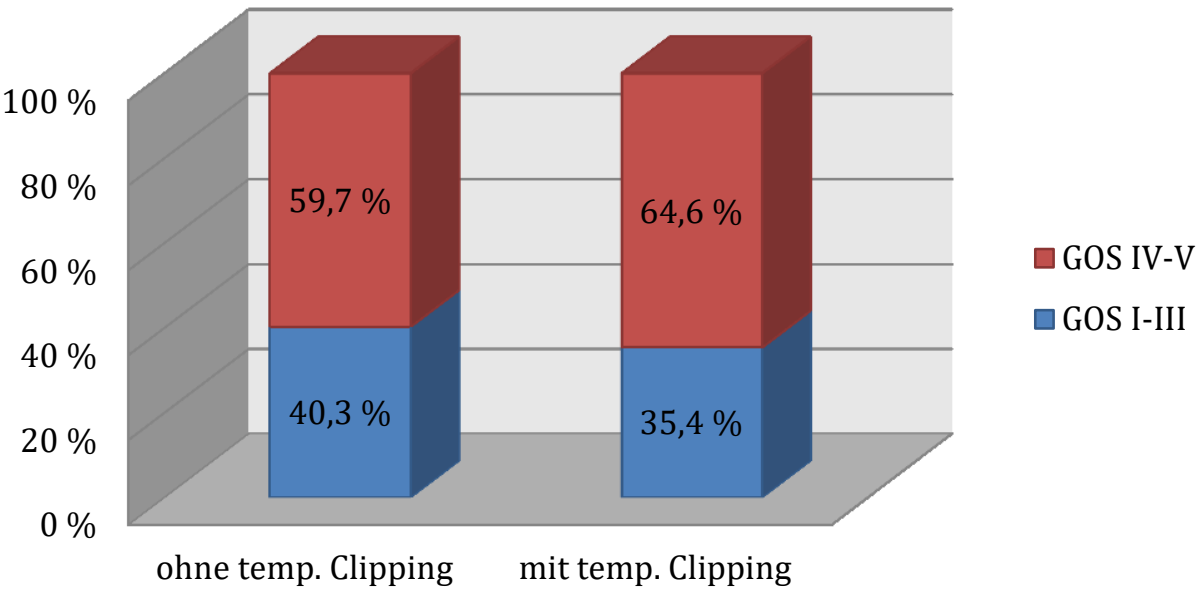

Abbildung 3-6: Vergleich des GOS bei Patienten mit und ohne temporäres Clipping

weder auf das Outcome des Patienten noch auf das Auftreten von DINDs eine signifikante Auswirkung hatte. Das Verhältnis innerhalb der Gruppen von Patienten mit und ohne temporäres Clipping war sehr ähnlich. So lässt sich beim GOS beobachten, dass in der Gruppe ohne temporäres Clipping 59,7\% der Patienten einen GOS von IV oder V aufwiesen. In der Gruppe mit temporärem Clipping waren es 64,6\% ( $\mathrm{p}=0$ 0,19; s. Abbildung 3-6).

Ein ähnliches Verhältnis trat bei der Untersuchung der DINDs auf $(21,5 \%$ vs. $26,2 \% ; p=0,26$; s. Abbildung 3-7).

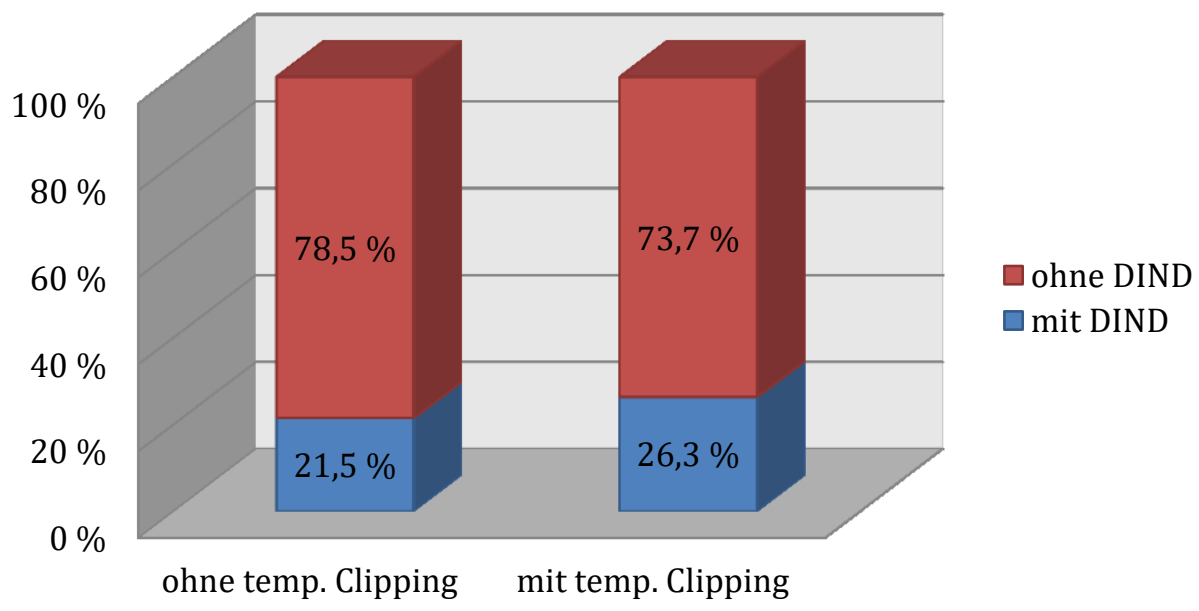

Abbildung 3-7: DIND-Aufkommen bei Patienten mit und ohne temporäres Clipping 
Auch beim Auftreten postoperativer TCD-Vasospasmen gab es vergleichbare, nicht signifikante Ergebnisse. Der Anteil an Patienten, die einen Vasospasmus entwickelten, war in beiden Gruppen in etwa gleich groß (59,3\% vs. 63,3 \%; $\mathrm{p}=0,39 ;$ s. Abbildung 3-9).

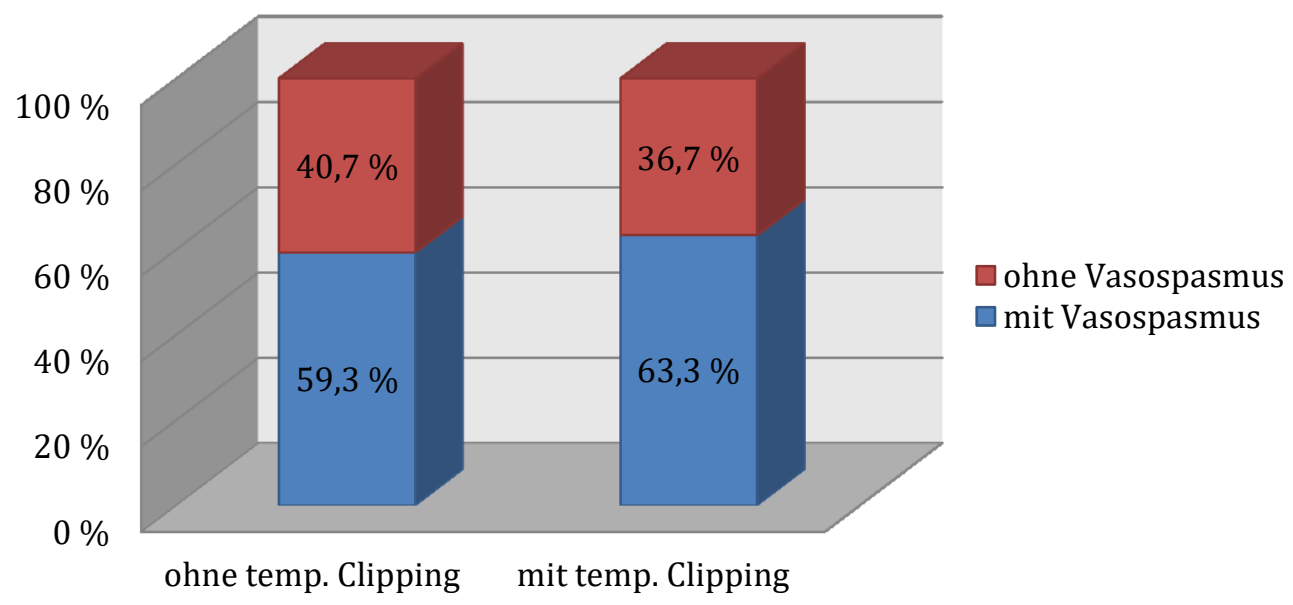

Abbildung 3-9: Vasospasmus-Aufkommen bei Patienten mit und ohne temporäres Clipping

Allerdings zeigte sich eine signifikante Korrelation zwischen temporärem Clipping und postoperativ aufgetretenen, Spasmus-induzierten Hirninfarkten $(p=0,02)$. Hier gab es wesentlich mehr Infarkte in der Gruppe ohne $(28,6 \%)$ als in der Gruppe mit temporärem Clipping (16,7 \%; s. Abbildung 3-8).

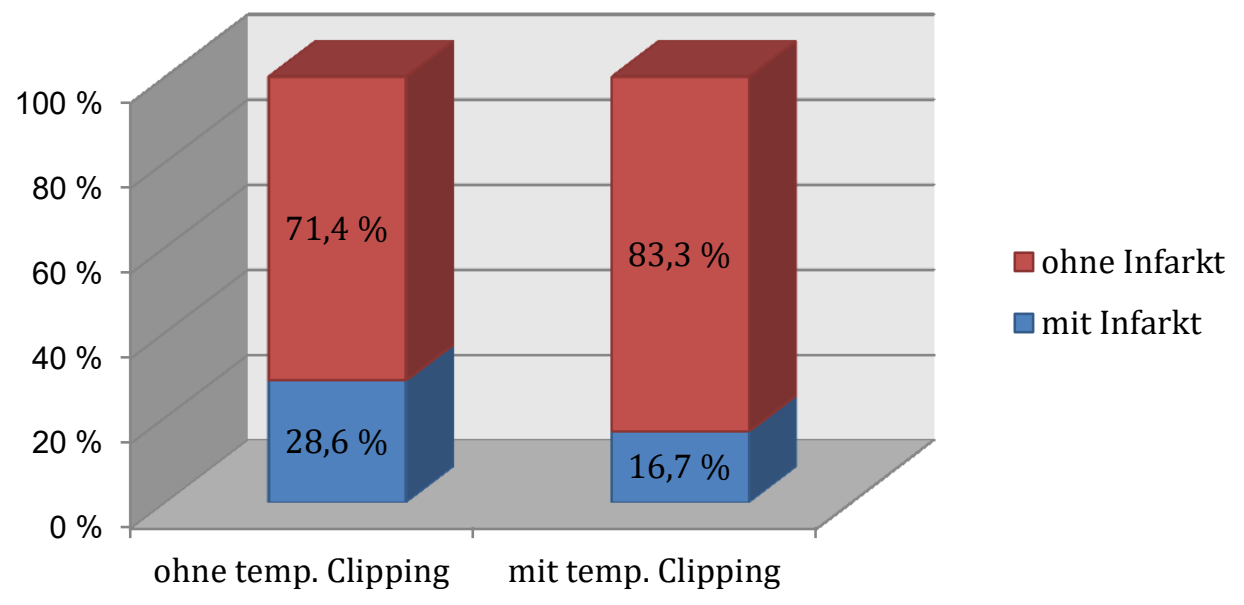

Abbildung 3-8: Aufkommen Spasmus-induzierter Infarkte bei Patienten mit und ohne temporäres Clipping 


\subsubsection{Multivariate Analyse}

Das Vorliegen eines postoperativen, zerebralen Vasospasmus' wurde mittels einer multivariaten logistischen Regressionsanalyse im Hinblick auf mögliche Einflussfaktoren untersucht. Im Zentrum der Untersuchung stand hierbei der Einfluss des temporären intraoperativen Gefäß-Clippings.

Es wurde ein binäres Logit-Model für die Regressionsanalyse erstellt. Grundlage bildete das Vorliegen oder Nicht-Vorliegen eines Vasospasmus kategorisiert in „nein“ (0) oder ,ja“ (1). Somit wurden zwei Werte in das Modell eingefügt (0 und 1). Insgesamt lagen zu 436 Patienten Angaben zum Vasospasmus und den untersuchten Variablen vor. Davon wurden 169 (38,8 \%) mit „nein“ und 267 (61,2 \%) mit ,ja“ kategorisiert.

Hauptvariable für die Regressionsanalyse war das stattgehabte intraoperative temporäre Clipping. Auch hier erfolgte eine Kategorisierung in „ja“ und „nein“. Als weitere Einflussfaktoren wurden der initiale GCS-Wert (dichotomisiert in (I): 3 12 und (II): 13 - 15), der Hunt und Hess-Grad (dichotomisiert in (I): 1 - 3 und (II): 4 - 5), der Fisher-Grad (dichotomisiert in (I): 1 - 2 und (II): 3 - 4), das Alter des Patienten (dichotomisiert in (I): $\leq 60$ Jahre und (II): > 60 Jahre), das Geschlecht des Patienten und der Zeitpunkt der Operation (dichotomisiert in (I): $\leq 3$ Tage nach SAB und (II): > 3 Tage nach SAB).

Es erfolgte die Berechnung der maximalen Wahrscheinlichkeitsschätzung (Maximum Likelihood Estimates) mit Hilfe von Regressionskoeffizient (B), Standardfehler und Wald-Chi-Quadrat-Test. Ebenso wurden Odds Ratios und 95\%-Wald-Konfidenz-Intervalle (KI) für die einzelnen Variablen bestimmt.

Unter Berücksichtigung der verschiedenen Einflussfaktoren, die konsekutiv mit dem Parameter Vasospasmus „nein“ oder „ja“ gegengerechnet wurden, zeigte sich, dass zwischen dem temporären Gefäß-Clipping und dem postoperativen Vasospasmus kein signifikanter Zusammenhang besteht $(\mathrm{p}=0,56)$. Gleiches gilt für die nachfolgend berechneten Variablen: initialer GCS-Wert $(p=0,83)$, Hunt und Hess-Grad der SAB $(\mathrm{p}=0,43)$ und der Ausdehnung der SAB nach Fisher $(\mathrm{p}=0,38)$. Odds Ratio und KI für diese Parameter zeigten ebenfalls, dass jeweils kein hoher Einfluss auf das Eintreten eines Vasospasmus zu erwarten ist: Temporäres Clipping 0,88 [0,57 -1,35]; GCS 1,07 [0,59 - 1,93]; Hunt und Hess 0,91 [0,72 - 1,15] und Fisher 0,87 [0,63 - 1,19]. 
Im Gegensatz dazu gab es signifikante Einflüsse durch das Alter des Patienten $(p<0,01)$, das Geschlecht $(p=0,02)$ und den Zeitpunkt der Operation nach Eintreten der SAB $(\mathrm{p}<0,01)$. Hier zeigten ebenfalls die Werte der Odds Ratio die Abhängigkeit besonders zwischen dem Alter der Patienten $(5,40$ [2,75 - 10,61]) und dem Zeitpunkt der Operation (2,49 [1,47 - 4,21]).

Die genauen Werte der logistischen Regressionsanalyse zeigt Tabelle 3-3.

\begin{tabular}{l|ccccccc}
\hline \multicolumn{1}{l}{ Parameter } & B & SE & Wald & $\mathbf{p}$ & OR & 95\% KI \\
\hline Temp. Clipping & -0.1274 & 0.2190 & 0.3385 & 0.5607 & 0.880 & 0.573 & 1.352 \\
GCS & 0.0643 & 0.3013 & 0.0455 & 0.8310 & 1.066 & 0.591 & 1.925 \\
H\&H & -0.0920 & 0.1159 & 0.6300 & 0.4273 & 0.912 & 0.727 & 1.145 \\
Fisher & -0.1417 & 0.1624 & 0.7610 & 0.3830 & 0.868 & 0.631 & 1.193 \\
Alter & 1.6872 & 0.3443 & 24.0176 & $<.0001$ & 5.404 & 2.752 & 10.612 \\
Geschlecht & -0.5103 & 0.2199 & 5.3863 & 0.0203 & 0.600 & 0.390 & 0.924 \\
OP-Zeitpunkt & 0.9101 & 0.2692 & 11.4256 & 0.0007 & 2.485 & 1.466 & 4.211 \\
\hline
\end{tabular}

Tabelle 3-3: Logistische Regressionsanalyse der angegebenen Parameter in Bezug auf das Vorliegen eines Vasospasmus.

B: Regressionskoeffizient B; SE: Standardfehler; Wald: Wald-Chi-Quadrat-Test; $p$ : Irrtumswahrscheinlichkeit; OR: Odds Ratio; KI: Konfidenzintervall; GCS: Glasgow Coma Scale; H\&H: Hunt und Hess-Grad 


\section{Diskussion}

\subsection{SAB allgemein}

Trotz eines verhältnismäßig geringen Vorkommens von SABs im Gesamtbild der Schlaganfallsformen (ca. 2 - $5 \%$ ) gilt diesem Krankheitsbild eine gesonderte Aufmerksamkeit. Dies liegt insbesondere daran, dass die SAB, verglichen mit anderen Unterformen des Schlaganfalls, eine extrem hohe Mortalität und Morbidität aufweist (s. Kapitel 1.1). Da die große Mehrzahl der Blutungen durch Ruptur eines Aneurysmas entsteht (ca. 85 \%; van Gijn et al. 2007; van Gijn und Rinkel 2001) und die Aneurysma-Behandlung nach aSAB Gegenstand dieser Arbeit ist, wird in der Folge nicht mehr auf die SAB anderer Ursachen wie z. B. tSAB eingegangen und die nachfolgenden Ausführungen beziehen sich bis auf Weiteres auf die aSAB.

Die Inzidenz einer SAB ist mit ca. 10/100000 Personen/Jahr angegeben, wobei es regionale Unterschiede gibt und z. B. in Regionen wie Japan oder Finnland eine deutlich höhere Inzidenz von bis zu 20/100000 Personen/Jahr vorliegt (Krex et al. 2001; Linn et al. 1996; Pobereskin 2001). Die Gründe für diese Unterschiede sind nicht bekannt. Frauen sind wesentlich häufiger betroffen als Männer, Linn et al. berichten ein 1,6-fach höheres Risiko bei Frauen (Linn et al. 1996). Dieses erhöhte Risiko scheint sich mit zunehmendem Alter zu verstärken (Rooij et al. 2007). Auch unser Patientenkollektiv wies eine deutliche Mehrheit bei weiblichen Patienten auf (65,6 \%). Die Prävalenz der SAB ist schwierig zu erörtern. Es gibt einige Studien, die eine Prävalenz intrakranieller Aneurysmen angeben und daraus das Risiko für eine Ruptur eines dieser Aneurysmen und eine damit einhergehende aSAB bestimmen. So wird die generelle Prävalenz mit ca. 3 - $5 \%$ angegeben (Dumont et al. 2002; Etminan et al. 2015), wobei sich in anderen Studien auch Schwankungen von $0,2 \%$ bis zu $9 \%$ zeigen (Rinkel et al. 1998; Winn et al. 2002). Die Gründe für unterschiedliche Ergebnisse liegen in der Art der Studien begründet. So stehen einerseits retrospektive, auf Autopsien basierende Studien, prospektiven, angiographisch geführten Studien gegenüber.

Das Risiko einer Ruptur wird mit ca. 0,5 - $2 \%$ pro Jahr angegeben, wobei es Abhängigkeiten von der Größe des Aneurysmas gibt. Die meisten der 
unrupturierten Aneurysmen (80 - 90 \%) haben eine Größe von bis zu 10 mm Durchmesser (Winn et al. 2002). Je größer der Durchmesser wird, desto größer wird auch die Gefahr einer Ruptur (Wiebers et al. 1987; Juvela et al. 2013; Rinkel et al. 1998; van Gijn et al. 2007). Ebenfalls ein deutlich erhöhtes Risiko haben Patienten mit unrupturierten Aneurysmen, wenn sie bereits einmal eine aSAB erlitten haben (ISUIA - International Study of Unruptured Intracranial Aneurysms Investigators 1998; Wiebers et al. 2003). Bei 20 - $30 \%$ der Patienten finden sich multiple Aneurysmen (Schievink 1997b; Wachter et al. 2011a). Dies deckt sich mit den Ergebnissen aus unserer Untersuchung. Auch in unserer Studie wurden bei 20,8 \% der Patienten multiple Aneurysmen diagnostiziert.

Aufgrund des Voranschreitens der diagnostischen Möglichkeiten in den letzten Jahren und der damit verbundenen häufigeren Diagnosesicherung intrakranieller unrupturierter Aneurysmen gibt es eine anhaltende Diskussion darüber, ob bzw. in welchen Fällen unrupturierte Aneurysmen therapiert werden sollten, um z. B. das Eintreten einer aSAB zu verhindern. So muss für jeden Patienten individuell entschieden werden, wie hoch das Risiko für eine Ruptur - andererseits aber auch das einer möglichen Intervention einzuschätzen ist (Etminan et al. 2015).

\subsubsection{Anatomische Grundlagen}

Die Schwere der Erkrankung liegt auch in den anatomischen Voraussetzungen begründet. Die für die Blutversorgung des Gehirns zuständigen Gefäße verlaufen im Subarachnoidalraum. Somit entstehen auch die Aneurysmen dieser Gefäße innerhalb des Subarachnoidalraumes, sodass sich bei einer Ruptur der Aneurysmen das Blut direkt innerhalb dieses Raumes verteilt.

Der Subarachnoidalraum befindet sich innerhalb der Leptomeninx encephali, der weichen Hirnhaut, die sich aus der Pia mater encephali, die direkt dem Hirnparenchym aufliegt, und der Arachnoidea encephali, die der äußeren (harten) Hirnhaut (Dura mater encephali) innen aufliegt, zusammensetzt. Zwischen der Pia mater und der Arachnoidea entspannt sich der Subarachnoidalraum, der auch als äußerer Liquorraum bezeichnet wird (s. Abbildung 4-1). Er gleicht die räumlichen Differenzen zwischen der zerfurchten Hirnoberfläche und der glatten Schädelkalotte durch die Bildung von sogenannten Zisternen aus, in denen sich bei 
Eintreten einer SAB vermehrt Blut sammelt. Kontakt zum inneren Liquorraum, den vier Hirnventrikeln, besteht über Öffnungen am vierten Ventrikel und über die Lamina terminalis am Vorderrand des dritten Ventrikels, damit eine ständige Liquorzirkulation aufrechterhalten werden kann. Über diese Verbindungen kann es im Zuge einer Subarachnoidalblutung auch zum Bluteintritt in das Ventrikelsystem - einer intraventrikulären Blutung - kommen. Zusätzlich kann ebenfalls eine Einblutung ins Hirnparenchym auftreten, womit in der Regel die Schädigung oder sogar Zerstörung des betroffenen Hirngewebes und, je nach betroffenem Hirnareal, entsprechende neurologische Defizite einhergehen.

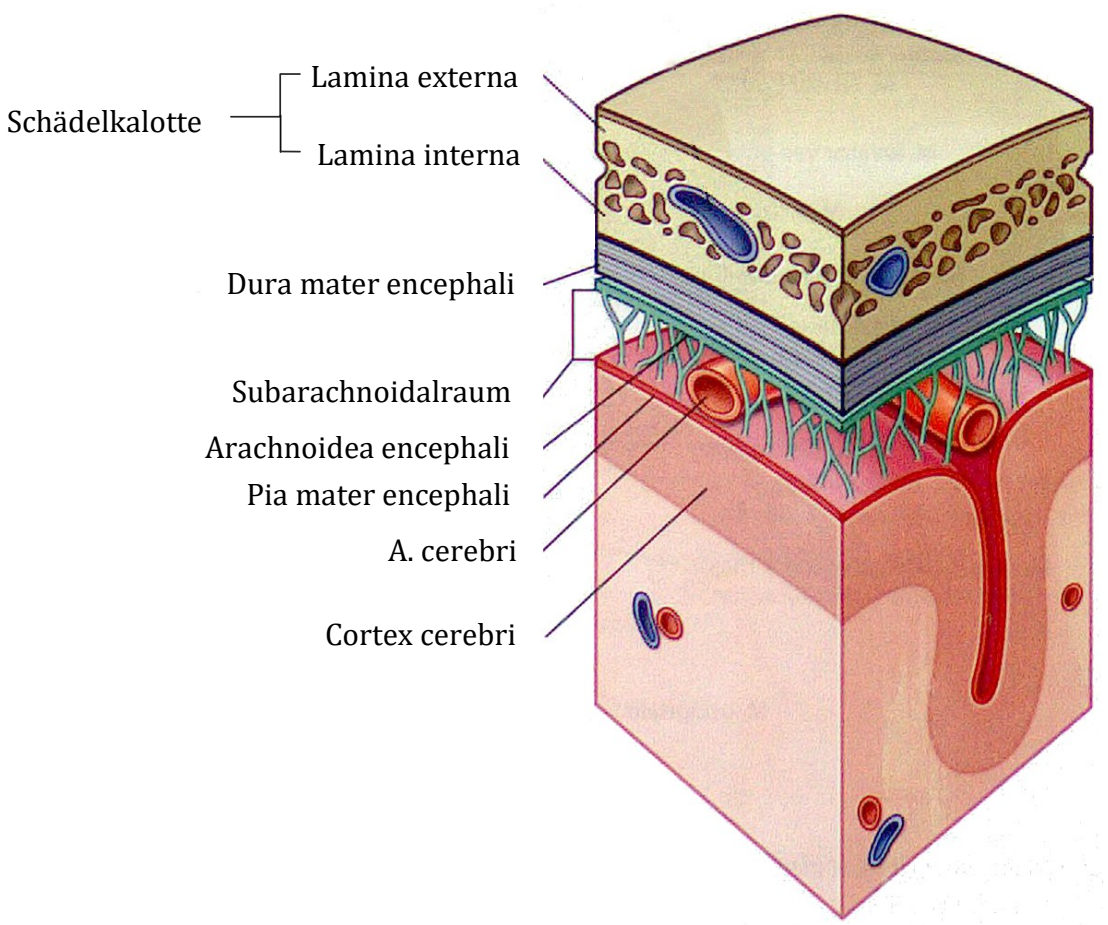

\section{Abbildung 4-1: Anordnung der Hirnhäute und deren Räume.}

(Modifiziert entnommen aus Drake et al. 2013, S. 442; die Verwendung erfolgt mit Genehmigung des Elsevier-Verlags.)

Die intrakraniellen Aneurysmen bilden sich an den Gefäßen des Circulus arteriosus Willisi (Greenberg 2001; Moskopp und Wassmann 2005; van Gijn et al. 2007). Dieser umfasst die paarig angelegten Arterien, die, an der Hirnbasis liegend, im vorderen Gebiet der A. carotis interna und im hinteren Stromgebiet der A. basilaris bzw. den $A a$. vertebrales entspringen. Die aus dem vorderen und hinteren Stromkreislauf hervorgehenden Arterien $A a$. cerebri mediae, anteriores und 
posteriores bilden durch die Aa. communicantes anteriores und posteriores einen Kreislauf, um eine durchgehende Blutversorgung des Gehirns zu sichern. Gerade Gefäßaufzweigungen (Bifurkationen) sind bevorzugte Stellen für die Ausbildung von Aneurysmen. Gründe dafür liegen unter anderem in den durch die Aufzweigung bedingten Veränderungen der Gefäßwandschichten, die eine Aussackung der Gefäßwand begünstigen. Zusätzlich sorgt eine Bifurkation für hämodynamische Änderungen des Blutdurchflusses innerhalb der Gefäße, welche ebenfalls die Entwicklung von Aneurysmen fördern können (Krex et al. 2001). Häufigkeitsverteilungen der Aneurysma-Lokalisationen zeigen, dass mehrheitlich im vorderen Stromgebiet des Circulus arteriosus Willisi Aneurysmen auftreten (80 - 95 \%; Greenberg 2001; Kassell et al. 1990a; Winn et al. 2002). Dazu passt auch die Verteilung in den Ergebnissen unserer Studie - hier befanden sich 86,8 \% der operierten Aneurysmen im anterioren Bereich (s. Abbildung 1-1).

\subsubsection{Risikofaktoren}

Die Entstehung zerebraler Aneurysmen und das Eintreten einer SAB durch deren Ruptur sind in der Regel auf Defekte und/oder Veränderungen in den Gefäßwandschichten zurückzuführen. Diese können angeboren oder erworben sein und werden durch bestimmte Risikofaktoren begünstigt. In einem umfassenden Review über 37 Studien mit insgesamt 3936 Fällen von SAB zeigten Feigin et al., dass vor allem Bluthochdruck, Rauchen und exzessiver Alkoholgenuss signifikante Einflüsse auf ein erhöhtes SAB-Risiko haben (Feigin et al. 2005).

Einer der wichtigsten Faktoren ist das Vorhandensein eines Hypertonus. Mehrere Studien zeigen, dass bei Patienten mit nicht rupturierten Aneurysmen, besonders aber bei Patienten mit eingetretener aSAB signifikant häufiger ein Bluthochdruck vorliegt als bei Patienten, bei denen kein Aneurysma nachgewiesen wurde. Das Risiko, bei einem nachgewiesenen, unrupturierten Aneurysma in der Folge eine aSAB zu erleiden, ist bei Patienten mit Bluthochdruck signifikant höher als bei vergleichbaren Patienten mit normalem Blutdruck (Isaksen et al. 2002; Sacco et al. 1984; Taylor et al. 1995).

Ein zusätzlich häufig angegebener Risikofaktor ist das Rauchen. Auch hier zeigen mehrere Studien, dass Zigarettenkonsum ebenso wie übermäßiger Alkoholgenuss eine aSAB begünstigen (Isaksen et al. 2002; Teunissen et al. 1996; Weir et al. 1998). 
Andere Ursachen wie genetische Faktoren oder familiäre Häufungen werden vermutet und untersucht. Schievink zeigte, dass das aSAB-Risiko bei Verwandten ersten Grades von Patienten mit erlittener aSAB bis zu vierfach höher ist und 7 - $20 \%$ der aSAB-Patienten auch Verwandte mit intrakraniellen Aneurysmen haben (Schievink 1997a). Neuere Studien zeigen, dass besonders für jene Personen ein hohes Risiko besteht, bei denen zwei oder mehr Verwandte ersten Grades betroffen sind. In solchen Fällen sollte ein Screening auf intraktranielle Aneurysmen durchgeführt werden, um das Risiko einschätzen zu können und ggf. eine aSAB zu verhindern (Bor et al. 2008; Bor et al. 2014; Vlak et al. 2011).

Ebenfalls als begünstigend für Gefäßmalformationen wie Aneurysmen und deren Rupturen werden Grunderkrankungen wie das Marfan- oder das Ehlers-DanlosSyndrom angesehen (Schievink et al. 1994).

In unserer Studie wurden für die Patienten der UMG ebenfalls Daten zu Vorerkrankungen erhoben. Auch hier zeigte sich, dass bei mehr als der Hälfte aller Patienten $(56,4 \%, \mathrm{n}=298)$ Vorerkrankungen bekannt waren und dabei in fast allen Fällen (94,1 \%) ein Hypertonus vorlag. Aufgrund fehlender Daten in der Aachener Patientensammlung wurden diese Feststellungen in die vorliegende Untersuchung nicht mit einbezogen.

\subsubsection{Intervention}

Zur Behandlung der aSAB und zum Verschluss des rupturierten Aneurysmas stehen hauptsächlich zwei interventionelle Therapiemöglichkeiten zur Verfügung. Lange Zeit bestand lediglich die Möglichkeit, das Aneurysma auf mikrochirurgischem Wege mittels Kraniotomie und Clipping auszuschalten und somit eine erneute Blutung zu verhindern. Seit Mitte der 1990er Jahre findet alternativ auch eine nicht-chirurgische Methode Anwendung - die von Guglielmi entwickelte Coiling-Therapie. Nach durchgeführter Diagnostik und genauer Lageund Formbestimmung des $\mathrm{zu}$ behandelnden Aneurysmas wird entschieden, welche Therapieform beim jeweiligen Patienten Anwendung findet.

Eine neue Entwicklung in der Aneurysma-Therapie stellt der sog. Flow diverter dar. Diese spezielle Form eines Stents wird vor allem bei der Behandlung von besonders großen (sog. giant aneurysms) oder breitbasigen Aneurysmen eingesetzt, die z. B. für ein Coiling nicht zugänglich sind oder anderweitig nur 
unzureichend ausgeschaltet werden können. Diese Stents aus engmaschigem Material bewirken eine Abänderung des Blutflusses innerhalb des Aneurysmalumens und stellen eine reguläre Gefäßdurchblutung wieder her. Zusätzlich werden durch die Flussveränderung thrombotische Prozesse innerhalb des Aneurysmasackes in Gang gesetzt und dadurch ein Verschluss des Aneurysmas bewirkt, während das Trägergefäß ganz normal durchblutet wird. Es wurde darüber hinaus gezeigt, dass sich entlang des Stents neue Gefäßwandschichten bilden, sodass eine Rekonstruktion des Trägergefäßes ermöglicht wird (D'Urso et al. 2011). Diese Art der Behandlung findet allerdings erst seit wenigen Jahren Anwendung und es fehlen Langzeit-Daten zur Beurteilung des dauerhaften Behandlungserfolges.

Alternative Behandlungsmethoden wie das sog. wrapping, bei dem das Aneurysma mitsamt dem zuführenden Gefäß durch Muskel-, Fasziengewebe oder synthetisches Material umhüllt und damit eine Blutstillung erreicht wird, oder das sog. trapping, durch welches ein Verschluss des zuführenden Gefäßes proximal und distal des Aneurysmas mittels Clips oder endovaskulären Ballons herbeigeführt wird (Moskopp und Wassmann 2005), finden nur selten Anwendung und werden im weiteren Verlauf nicht erörtert.

\subsubsection{Coiling}

Bei der endovaskulären Behandlungsmethode ist keine chirurgische Intervention (Kraniotomie) notwendig. Mit Hilfe von Mikrokathetern wird ein spiralförmiger Platin-Draht - ein sogenanntes Coil - durch die zuführenden Gefäße bis zum Aneurysma vorgebracht. Nun führt man die Platin-Spirale durch den Aneurysmahals ins Innere des Aneurysmas und legt so viele Coils ein, bis das Aneurysma komplett ausgefüllt ist (s. Abbildung 4-2). Anschließend wird mit niedrigen Stromstärken eine elektrische Ladung auf die Coils gebracht, durch die eine Spannung zu den ebenfalls elektrisch geladenen Thrombozyten entsteht. Die Ladung wird auch genutzt, um das Coilpaket nach korrekter Platzierung im Aneurysmasack vom Mikrokatheter abzulösen. Der Kontakt zwischen Blut und Coil bewirkt elektrothrombotische Prozesse, sodass sich im Aneurysmalumen um die eingelegten Coils ein Thrombus bildet, der die Blutung stoppt und das Aneurysma 
verschließt (Guglielmi et al. 1991). Die Lage der Coils sowie die Vollständigkeit des Aneurysmaverschlusses werden neuroradiologisch kontrolliert (s. Abbildung 4-3).

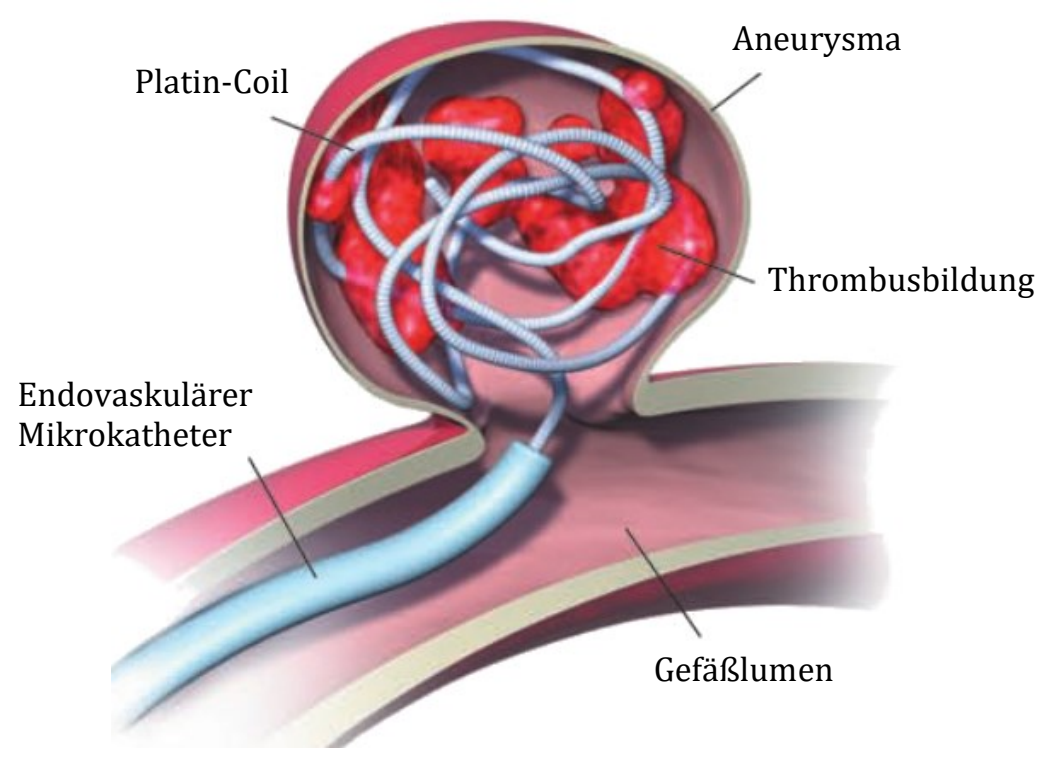

Abbildung 4-2: Schematische Darstellung eines Aneurysma-Verschlusses mittels Coiling.

(Modifiziert entnommen aus Suarez et al. 2006; die Verwendung erfolgt mit Genehmigung von Dr. J. Suarez, Copyright Massachusetts Medical Society.)
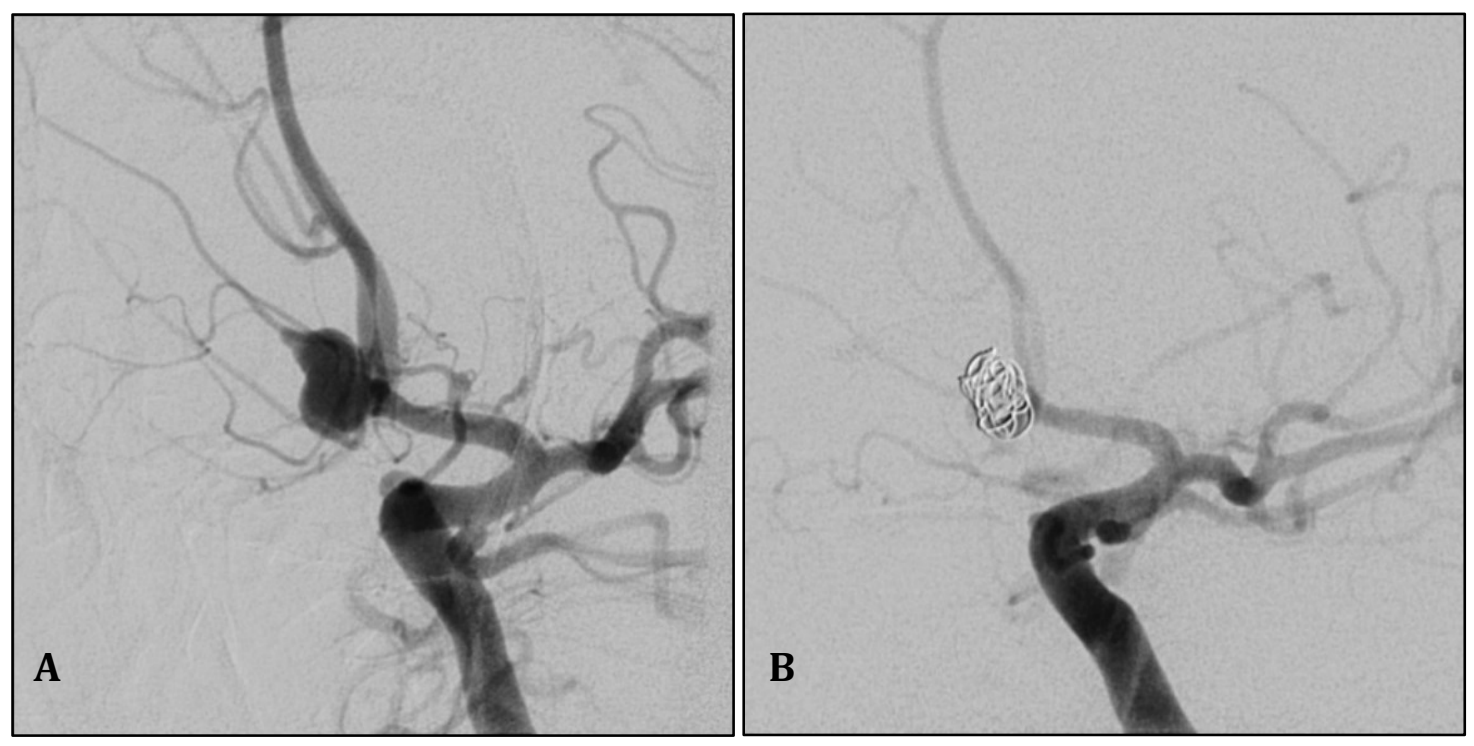

Abbildung 4-3: DSA eines AComA-Aneurysmas.

Zustand vor [A] und nach [B] Coiling. (Zur Verfügung gestellt vom Institut für Diagnostische und Interventionelle Neuroradiologie, UMG.) 


\subsubsection{Clipping}

Ist eine chirurgische Intervention angezeigt, so kommt es auf die Lage des Aneurysmas und des zuführenden Gefäßes an, welche Kraniotomie gewählt wird (s. Kapitel 1.3.1). Nach Darstellung des Aneurysmas über den jeweiligen Zugang wird dieses durch einen Titan-Clip am Hals verschlossen und so eine erneute Ruptur verhindert (s. Abbildung 4-4; Abbildung 4-5 A und B).

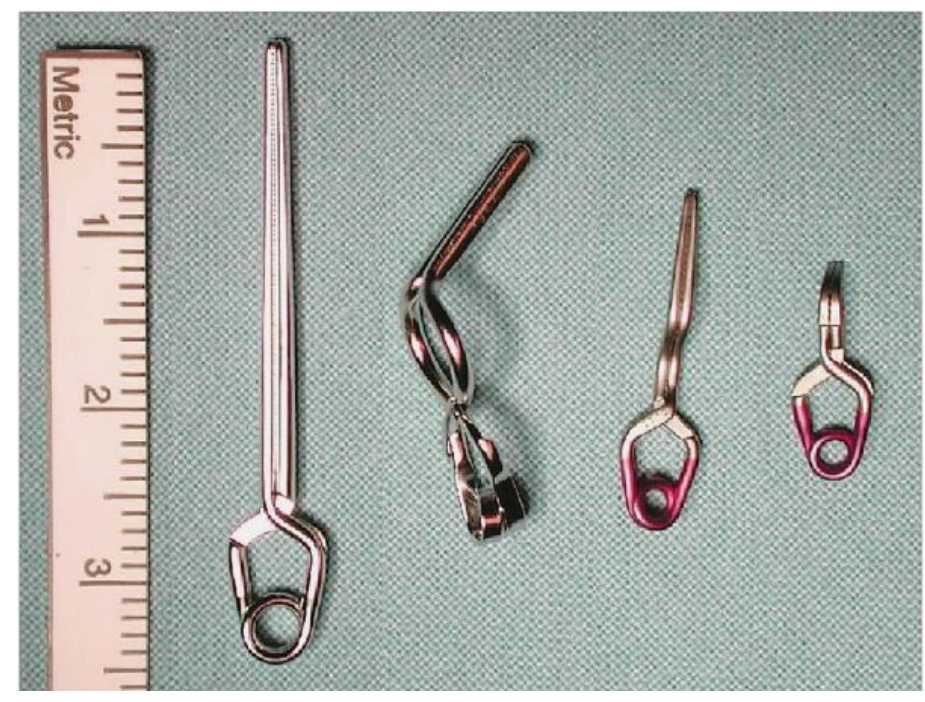

\section{Abbildung 4-4: Titan-Clips verschiedener Form und Größe}

(entnommen aus Ammerer et al. 2003; die Verwendung erfolgt mit Genehmigung des Verlags Krause \& Pachernegg GmbH.)

Das operierte Gefäß und auch der umgebende Bereich werden intraoperativ regelmäßig mit Nimodipin gespült, um Vasospasmen durch Manipulation der entsprechenden Gefäße zu reduzieren. Zusätzlich erfolgen intraoperativ nach Anlegen der Clips ICG-Angiographien oder Mikrodoppler-Sonographien zur Kontrolle des Blutflusses in allen beteiligten Gefäßen, um eine akzidentelle Okklusion von Perforatoren oder Trägergefäßen auszuschließen (s. Abbildung 4-5 C). Zudem soll eine Restperfusion bedingt durch ein sog. Dog-Ear-Remnant (Restaneurysma) ausgeschlossen werden (Siasios et al. 2012). Somit können Risiken minimiert werden, dass es im weiteren Verlauf zu Komplikationen wie erneuten Blutungen aus inkomplett verschlossenen Aneurysmen oder neurologischen Schäden durch Minderdurchblutung umgebender Hirnareale kommt (Mielke et al. 2014; Washington et al. 2013). 

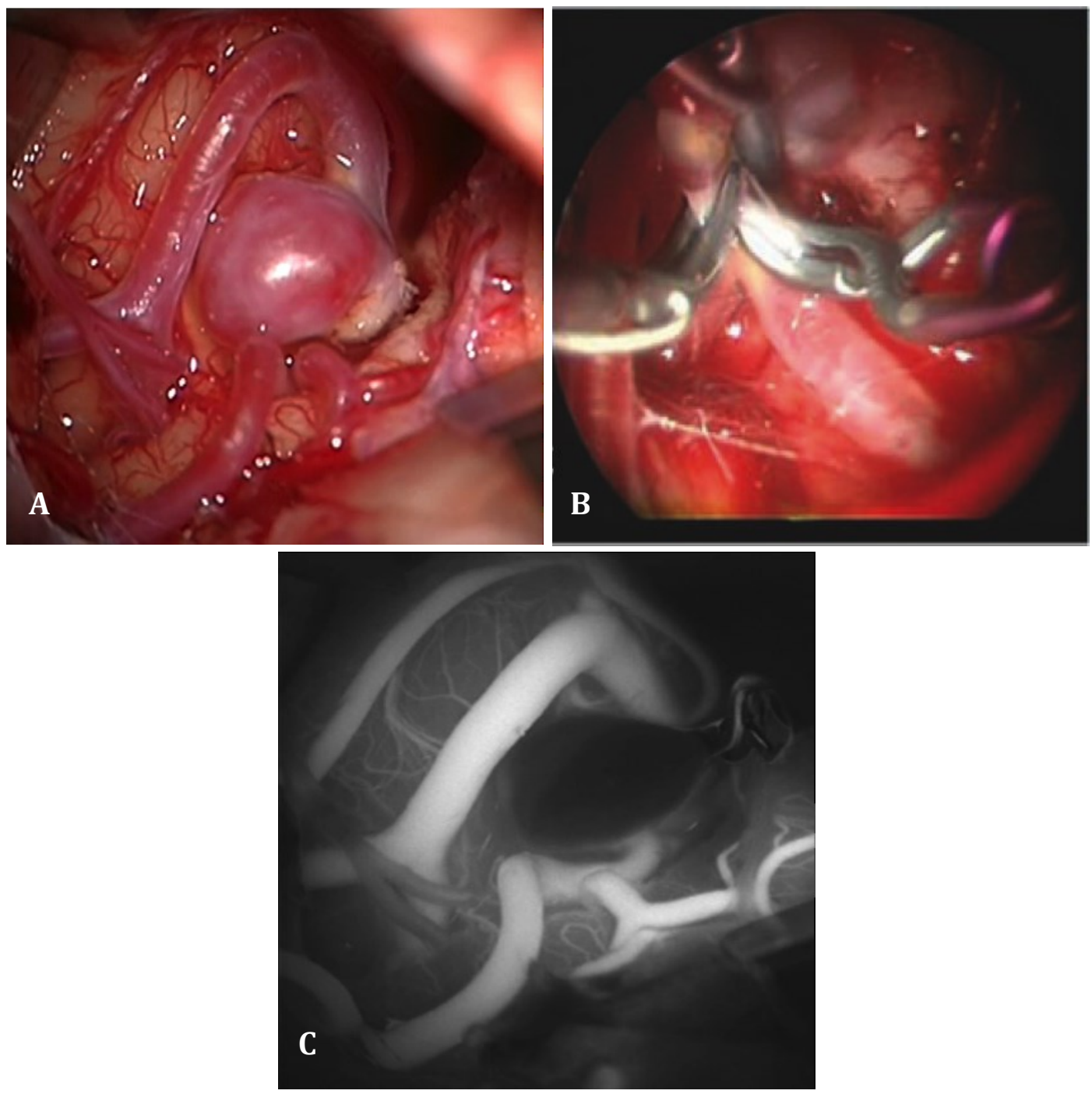

Abbildung 4-5: Intraoperative Aufnahme eines Aneurysmas.

Zustand vor [A] und nach [B] Clipping. [C] ICG-Angiographie nach Clip-Anlage ohne Restperfusion des Aneurysmas. (Zur Verfügung gestellt von Prof. Dr. D. Mielke, Klinik für Neurochirurgie, UMG.)

\subsubsection{Coiling vs. Clipping}

Bei der Betrachtung unserer Studie zeigt sich, dass innerhalb des Studienzeitraumes von 23 Jahren (1990 bis 2013) eine deutliche Verschiebung der eingesetzten Therapien stattgefunden hat. So ist innerhalb der Aachener Gruppe zu erkennen, dass aufgrund der im Laufe der 1990er Jahre noch nicht so weit verbreiteten Coiling-Technik beinahe $90 \%$ aller Patienten mikrochirurgisch mit Aneurysma-Clips versorgt wurden (398 von 456 Patienten). Hingegen ist im späteren Verlauf der Studie (2005 bis 2013) in der UMG mehr als die Hälfte aller Patienten durch Coiling behandelt worden (162 von 299 Patienten, 54,2 \%). Dementsprechend sank auch die Zahl der Patienten, die intraoperativ ein 
temporäres Clipping erfahren haben, mit der Zahl der generell chirurgisch versorgten Patienten. Waren es in der Aachener Klinik noch 40,5 \% aller ClippingPatienten, bei denen auch temporär Gefäße verschlossen wurden, waren es in Göttingen lediglich noch 13,9\%.

Im Laufe der Jahre, seit der Etablierung des Coilings, wird immer wieder diskutiert, ob die - langjährig bevorzugte und erprobte - Methode des mikrochirurgischen Clippings oder das Coiling bessere Behandlungsergebnisse für die Patienten bieten. In einer groß angelegten Studie wurden im Jahr 2005 Ergebnisse präsentiert, die einen direkten Vergleich zwischen den beiden Behandlungsmethoden ermöglichten. Im Zuge dieses International Subarachnoid Aneurysm Trial (ISAT, Molyneux et al. 2005) wurden 2143 Patienten untersucht, die eine aSAB erlitten haben und nach bestimmten Randomisierungskriterien entweder durch Clipping oder durch Coiling therapiert wurden. Die Studie ergab, dass bei Patienten mit endovaskulärem Aneurysmaverschluss das Risiko für ein schlechtes Outcome (Tod oder schwere Behinderung) um $7 \%$ geringer ist als bei chirurgisch versorgten Aneurysmen. Dieses zeigte sich auch im weiteren Verlauf ein Jahr nach der Erkrankung und blieb über den Zeitraum von 7 Jahren bestehen.

Allerdings muss zu dieser Untersuchung erwähnt werden, dass die in die Studie einbezogenen Patienten größtenteils in gutem klinischen Zustand nach Eintreten der SAB behandelt wurden (88 \% mit WFNS-Grad I oder II). Die meisten behandelten Aneurysmen befanden sich gut zugänglich im vorderen Stromgebiet des Circulus arteriosus Willisi (95 \%) mit einer kleinen Größe (90\% aller Aneurysmen hatten einen Durchmesser $<10$ mm). Für diese Art von Aneurysmen, die für beide Therapiemöglichkeiten gleichermaßen zur Verfügung stehen, scheint eine endovaskuläre Therapie bessere Ergebnisse zu erzielen. Die Daten zeigten aber auch, dass es in der Gruppe der chirurgisch behandelten Patienten eine deutlich höhere Anzahl an Nachblutungen im Vorfeld der Intervention gab als in der Gruppe der Coiling-Patienten, wodurch es in diesen Fällen sicher auch zu einer Verschlechterung des Outcomes kam. So stellten Molyneux et al. in der Auswertung ihrer Analyse fest, dass bei Ausschluss der Patienten, die durch eine präoperative Nachblutung verstorben sind, die Mortalität nach zwei Monaten in beiden Gruppen in etwa gleich hoch war (Molyneux et al. 2005). Darüber hinaus ergab die Studie allerdings auch, dass mit Coils versorgte Aneurysmen ein höheres Risiko für 
postinterventionelle Nachblutungen haben und bei angiographischer Kontrolle eine geringere Verschlussrate haben als durch Clips verschlossene Aneurysmen. Bezüglich der Verschlussrate und den Nachblutungen kommen McDougall et al. in ihrer Studie, dem Barrow Ruptured Aneurysm Trial (BRAT, McDougall et al. 2012), zu ähnlichen Ergebnissen. Auch hier wurden Patienten mit Coiling und Clipping gegenübergestellt. Im Gegensatz zur ISAT-Studie war hier das Ziel, alle aSABPatienten unabhängig von Lage und Größe des Aneurysmas einzubeziehen, um den Einfluss der Behandlungsart zu analysieren. Die 6-Jahres-Daten dieser Studie zeigten, wenn auch mit deutlich geringeren Fallzahlen als beim ISAT, dass zwischen den beiden Gruppen kein signifikanter Unterschied in Bezug auf ein schlechteres Outcome festzustellen ist, wenn man alle behandelten Aneurysmen betrachtet. Allerdings stellte sich bei der Analyse der Aneurysma-Lokalisation heraus, dass für Aneurysmen im posterioren Stromgebiet ein besseres Outcome durch Coiling erreicht werden kann, wohingegen bei anterior gelegenen Aneurysmen kein Unterschied zwischen beiden Methoden ersichtlich war (Spetzler et al. 2015).

Letztendlich muss die Entscheidung zwischen den beiden Therapien abhängig vom Gesamtzustand des Patienten, der Lage und Größe des Aneurysmas und den in der jeweiligen Klinik zur Verfügung stehenden Möglichkeiten individuell erörtert werden (van der Schaaf et al. 2005).

Sandström et al. zeigten, dass bei Patienten in schlechtem klinischen Zustand (WFNS-Grad IV - V) kein signifikanter Unterschied im Outcome der Patienten zwischen Coiling und Clipping festzustellen ist und beide Methoden in der richtigen Anwendung gute Ergebnisse erzielen können (Sandström et al. 2013).

Neuere Studien belegen, dass auch für postoperative Komplikationen keine signifikanten Unterschiede zwischen Coiling und Clipping ausschlaggebend sind, beispielsweise in Bezug auf Vasospasmen oder zerebrale Infarkte (Jones et al. 2015; Kanamaru et al. 2015). Eine umfangreiche Meta-Analyse von Li et al. dagegen weist auf erhöhtes Auftreten von Vasospasmen nach Clipping hin, konnte dies bei Infarkten allerdings nicht nachweisen (Li et al. 2013).

In unserer Datenbank aller Aachener und Göttinger aSAB-Patienten (Therapie durch Clipping oder durch Coiling) lässt sich ablesen, dass ein größerer Anteil an Patienten, die mit Coiling therapiert wurden, ein schlechteres Outcome verglichen mit operierten Patienten aufwiesen (GOS I oder II, $33 \%$ bei Coiling, $16 \%$ bei 
Clipping). Ebenso zeigt sich, dass Patienten mit Clipping häufiger in gutem Zustand entlassen wurden (GOS IV oder V, 47 \% bei Coiling, 61 \% bei Clipping). Zu diesen Ergebnissen passt allerdings auch, dass die Schwere der aSAB bei den operierten Patienten anteilig geringer war als bei den Coiling-Patienten. So wurden 69,7 \% der Clipping-Patienten mit einer aSAB vom Hunt und Hess-Grad I - III eingeliefert. Lediglich 30,3 \% wiesen eine schwere aSAB (H\&H IV oder V) auf. Bei den endovaskulär behandelten Patienten liegt eine deutliche Verschiebung zur schweren aSAB vor (57,8 \% mit H\&H I - III; 42,2 \% mit H\&H IV oder V). Ein Vergleich zwischen Clipping und Coiling ist allerdings nicht Gegenstand dieser Studie gewesen und es wurden dahingehend keine Analysen durchgeführt. Die Patienten, die in unserer Datenbank gesammelt wurden, sind nicht nach Kriterien aufgenommen worden, die eine detaillierte und aussagekräftige Untersuchung diesbezüglich ermöglichte, sodass dies hier lediglich als deskriptive Einschätzung ohne eine genaue statistische Einordnung aufgeführt wird.

\subsection{Temporäres Gefäß-Clipping}

Ein großes Risiko im Zuge des Aneurysma-Clippings ist die intraoperative Ruptur des Aneurysmas. Diese Komplikation ist neben dem postoperativen Vasospasmus eine der Hauptgefahren bei der Behandlung und hat ebenfalls deutliche Auswirkungen auf das Outcome des Patienten. Angaben zur Häufigkeit dieser Komplikation schwanken in der Literatur stark zwischen unter $10 \%$ bis hin zu $40 \%$. Batjer und Samson zeigten in ihrer Studie mit über 300 Patienten eine Häufigkeit von 19 \% (Batjer und Samson 1986), Schramm und Cedzich berichteten von bis zu 40 \% (in Abhängigkeit vom Zeitpunkt der Operation nach Eintreten der SAB; Schramm und Cedzich 1993). In einer sehr umfangreichen Studie von Leipzig et al. mit 1269 Patienten wurde eine Rate von lediglich 6,7 \% intraoperativer Ruptur erreicht; sie führten den Rückgang im Vergleich zu älteren Studien unter anderem auf sicherere OP-Methoden, beispielsweise durch den Einsatz verbesserter OP-Mikroskope, zurück (Leipzig et al. 2005). Trotz aller Schwankungen in der Häufigkeit sind die mit einer Ruptur einhergehenden Konsequenzen in allen Studien gleichermaßen mit deutlich erhöhter Mortalität und Morbidität benannt. 
Der Einsatz temporärer Gefäßclips (s. Abbildung 4-6) bietet eine Möglichkeit, das Risiko einer intraoperativen Ruptur zu senken und damit ein besseres Outcome zu ermöglichen. Seit Beginn der breiten und regelmäßigen Anwendung des temporären Clippings in den 1960er und 1970er Jahren durch Pool und Suzuki (Pool 1961; Suzuki et al. 1979) wurde immer wieder dessen Einfluss auf Komplikationen - insbesondere die intraoperative Ruptur - untersucht und gezeigt, dass die temporäre Gefäßausschaltung ein probates Mittel zur Reduzierung des Rupturrisikos und der damit verbundenen Verschlechterung des Outcomes ist (Batjer und Samson 1986; Charbel et al. 1991; Dhandapani et al. 2013; Leipzig et al. 2005).

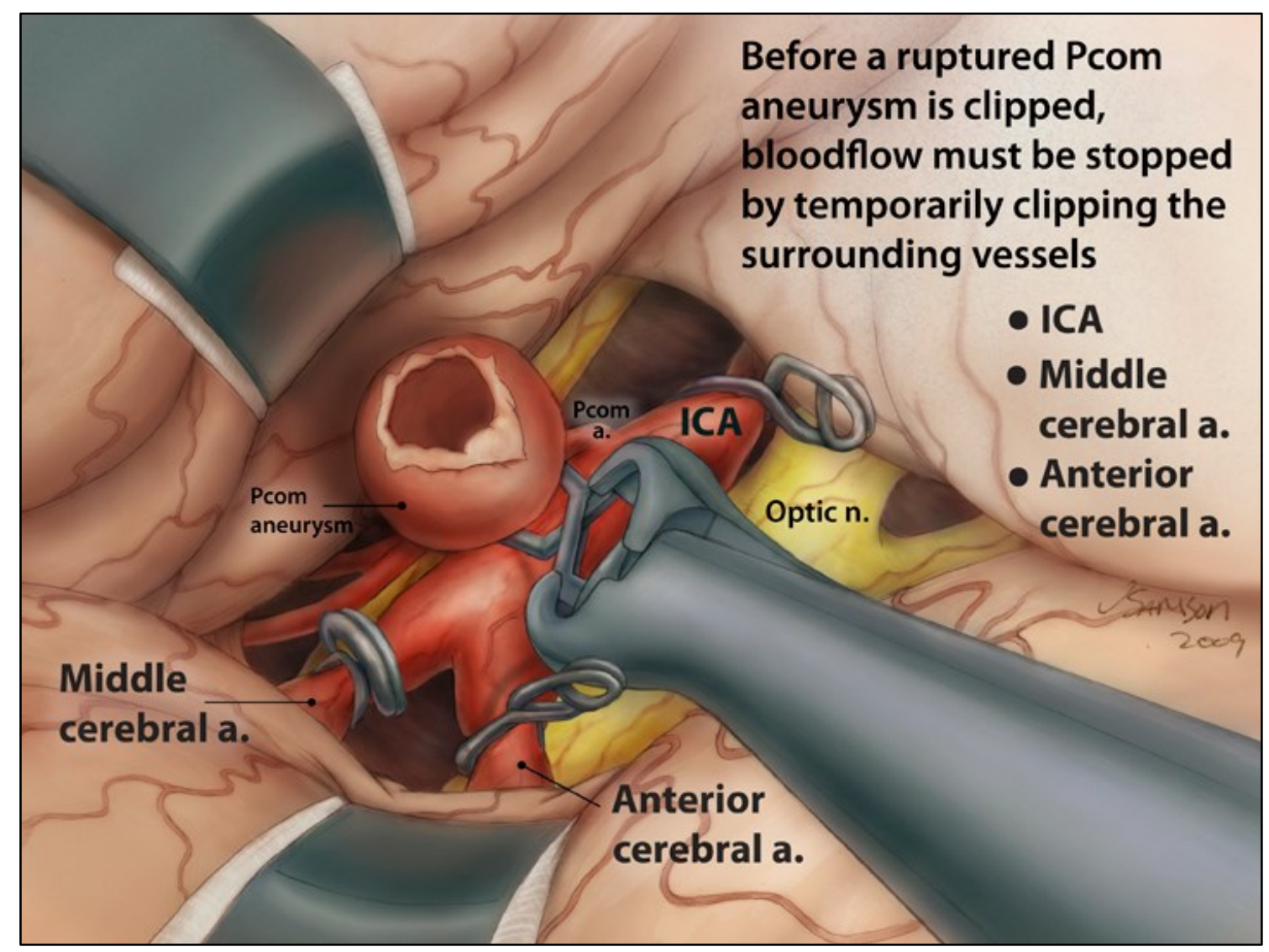

Abbildung 4-6: Zeichnerische Darstellung eines temporären Clippings.

Clipping eines PComA-Aneurysmas nach Anbringen von drei temporären Gefäßclips an den umliegenden Gefäßen MCA, ACI und ACA.

Illustration von Joseph Samson (C 2009). Die Verwendung erfolgt mit freundlicher Genehmigung von J. Samson, Department of Art as Applied to Medicine, Johns Hopkins University, School of Medicine, Baltimore, Maryland, USA. 
Zusätzlich ermöglicht temporäres Clipping eine Reduzierung des Blutdrucks im Bereich des Aneurysmas, sodass beispielsweise bei kompliziert liegenden oder besonders groß ausgeprägten Aneurysmen (giant aneuryms) eine bessere Möglichkeit zum Verschluss des Aneurysmahalses gegeben ist. Die zeitweise Ausschaltung des zuführenden Gefäßes lässt außerdem das Entfernen von arteriosklerotischer Plaque aus dem Bereich der Gefäßmalformation und eine gute Rekonstruktion des Trägergefäßes zu (Griessenauer et al. 2014; Taylor et al. 1996). Gut untersucht ist mittlerweile der Zusammenhang zwischen der Dauer des temporären Clippings und den intra- bzw. postoperativen Folgen, sodass generell empfohlen wird, mit dem Ausschalten der zuführenden Gefäße eine Zeitdauer von 15 bis 20 Minuten nicht zu überschreiten, um keine neurologischen Schäden hervorzurufen (Griessenauer et al. 2014; Samson et al. 1994). Hierbei wird allerdings auch diskutiert, ob mehrmaliges temporäres Clipping bei zwischenzeitlicher Reperfusion des Gefäßes ebenfalls Einflüsse hat. Dhandapani et al. zeigten in ihrer Studie, dass auch bei wiederholten Clipping-Episoden bis zu 20 Minuten kein Einfluss auf das Outcome gegeben ist, wohingegen längere Ausschaltzeiten durchaus eine Verschlechterung bedingen können (Dhandapani et al. 2013).

Unsere Studie wies in diesem Zusammenhang ähnliche Ergebnisse auf. Bei Patienten mit temporärem Clipping konnte kein signifikanter Einfluss auf das Outcome $(p=0,19)$ oder das Auftreten von DINDs $(p=0,26)$ ermittelt werden. In unseren Untersuchungen fand die Dauer der jeweiligen intraoperativen Gefäßausschaltung allerdings keine Berücksichtigung, sodass diesbezüglich keine Analyse auf den postoperativen Zustand möglich war. Ebenfalls wurden keine Informationen darüber gesammelt, ob intraoperativ nur einmalig oder mehrmals temporäre Clips angelegt wurden.

\subsection{Zerebraler Vasospasmus}

Die Entwicklung zerebraler Vasospasmen ist nach wie vor ein grundlegendes Problem in der Behandlung von SABs. Als einer der häufigsten Komplikationen nach SAB und einem der Hauptgründe für ein vermindertes Outcome (Kassell et al. 1990a) gilt dem Vasospasmus seit langem eine besondere Aufmerksamkeit im Konzept der SAB-Therapie, und eine gezielte Behandlung zur Vorbeugung oder 
Verringerung der Spasmen ist eines der vorrangigen Ziele nach erfolgreichem Aneurysmaverschluss.

Als Vasospasmus bezeichnet man eine pathologische Verengung eines Gefäßes. Eine Gefäßverengung führt automatisch $\mathrm{zu}$ einer Erhöhung der Blutflussgeschwindigkeit innerhalb des Gefäßes. Dies ist gerade bei zerebralen Gefäßen von hoher Bedeutung, da eine erhöhte Flussgeschwindigkeit eine Minderung der Sauerstoffversorgung der umgebenden Gewebe bedingen kann. Eine so entstehende Unterversorgung des Hirngewebes kann zu Infarkten und damit einhergehenden schwerwiegenden neurologischen Schädigungen führen. Das Auftreten zerebraler Vasospasmen im Zuge einer Subarachnoidalblutung ist nach wie vor sehr bedeutsam in der Therapie und dem Ausgang der Erkrankung. Sie sind neben Nachblutungen hauptverantwortlich für eine hohe Morbidität und Mortalität nach erfolgter Aneurysmaversorgung (Dorsch und King 1994; Kassell et al. 1990a). Bei bis zu 70 \% aller SAB-Patienten treten Vasospasmen auf (Biller et al. 1988; Crowley et al. 2011; Dorsch und King 1994; Torbey et al. 2001; Weir 1995). Auch in unserer Studie waren 61,4 \% aller Patienten nach dem AneurysmaClipping von Spasmen betroffen. Häufig führen Therapieversuche, die auf den Vasospasmus zielen, lediglich zur Minderung und nicht zum Ausschalten desselben, sodass trotz Therapie das Eintreten von Hirnschädigungen und damit verbundenen neurologischen Defiziten nicht verhindert werden kann.

Ursachen der Vasospasmen sind vielfältig, in der Literatur seit langem diskutiert und letztlich noch nicht vollends aufgeklärt. Als gesichert gilt, dass sowohl Bestandteile des durch die Aneurysmaruptur entstehenden extravasalen Blutgerinnsels als auch einzelne Stoffe der defekten Gefäßwand dazu führen, dass sich das betroffene Gefäß kontrahiert. Angeführt werden als hervorrufende Substanzen u.a. Hämoglobin, Endothelin, fehlendes Stickstoffmonoxid (NO) und eine erhöhte Reaktion der glatten Muskelzellen innerhalb der Gefäßwand auf freie Calcium-Ionen $\left(\mathrm{Ca}^{2+}\right)$, durch die direkt oder indirekt eine Vasokonstriktion hervorgerufen wird (Cossu et al. 2014; Pluta et al. 2009; Weir 1995). Ebenfalls wird darüber diskutiert, ob eine immunologische und/oder infektiöse Reaktion der Gefäßwände mit der Entstehung der Vasospasmen in Verbindung steht (Lin et al. 2014). 


\subsubsection{Risikofaktoren}

Gegenstand vieler Untersuchungen bisher war es, gewisse Risikofaktoren für die Entwicklung eines Vasospasmus aufzuzeigen. Es zeigte sich, dass vor allem ein schlechter klinischer Zustand nach Eintreten der SAB bzw. die Schwere und das Ausmaß der SAB deutlichen Einfluss haben.

Die Menge des im Subarachnoidalraum angesammelten Blutes erwies sich in vielen Studien als signifikanter Faktor bei Auftreten von Vasospasmen. Beurteilt nach dem Fisher-Grad haben Patienten mit großen Blutansammlungen und/oder intraventrikulären oder intrazerebralen Einblutungen (Grad III - IV) ein hohes Risiko für die Entwicklung postoperativer Vasospasmen (Frontera et al. 2009; Gonzalez et al. 2007; Macdonald et al. 2003; Rabb et al. 1994; Reilly et al. 2004; Treggiari-Venzi et al. 2001). Ebenso ist ein schlechter neurologischer Ausgangszustand nach Eintreten der SAB ein Faktor, der das Auftreten von Vasospasmen begünstigt. So haben Patienten mit hohem Hunt und Hess-Grad (IV - V) oder WFNS-Grad (IV - V) ein erhöhtes Risiko, Vasospasmen zu entwickeln (Dumont et al. 2010; Gonzalez et al. 2007; Inagawa et al. 2014; Macdonald et al. 2003).

Damit deckt sich, dass auch unsere Studie bei Analyse des direkten Einflusses auf den Vasospasmus eine Signifikanz bei hohem Fisher-Grad (III oder IV, p = 0,004, Chi-Quadrat-Test) und schlechtem neurologischen Zustand nach GCS (GCS 3 - 12 entsprechend WFNS-Grad IV - V; p = 0,04, s. Kapitel 3.2.6) zeigte. In unserem Fall beeinflusste dagegen der Hunt und Hess-Grad den Vasospasmus nicht signifikant $(\mathrm{p}=0,08)$ in der direkten Analyse.

Allerdings zeigte sich in der logistischen Regressionsanalyse in Bezug auf den Vasospasmus bei temporärem Clipping, dass weder der initiale GCS-Wert, der Hunt und Hess-Grad noch der Fisher-Grad eine Signifikanz für das Auftreten eines Vasospasmus aufwiesen (GCS: $p=0,83$; H\&H: $p=0,43$; Fisher: $p=0,38$, s. 3.2.8). Gründe dafür können darin liegen, dass innerhalb dieser multivariaten Analyse andere Faktoren durch ihre sehr starke Signifikanz (z.B. Alter, p < 0,0001; OP-Zeitpunkt, $\mathrm{p}=0$,0007) ein Absinken der Signifikanz der restlichen Faktoren bedingten. 
Viel diskutiert wird im Zusammenhang mit dem Vasospasmus auch das Alter der Patienten. Häufig ist in Studien zu beobachten, dass junge Patienten deutlich öfter an Vasospasmen leiden und das Risiko mit zunehmendem Alter abnimmt (Boecher-Schwarz et al. 1994; Charpentier et al. 1999; Magge et al. 2010; Rabb et al. 1994; Torbey et al. 2001; Wachter et al. 2011b). Wie allerdings auch Lanzino et al. und Gonzalez et al. zeigten, gibt es ebenso Studien mit hohen Fallzahlen, deren Analysen keinen signifikanten Einfluss des Alters auf den Vasospasmus nachweisen (Lanzino et al. 1996; Gonzalez et al. 2007). Genaue Ursachen für diese schwankenden Ergebnisse sind bisher nicht eindeutig herausgefunden worden. Eine Abnahme der Gefäßreaktivität auf äußere Faktoren bei einigen Patienten im höheren Alter sowie eventuelle Einflüsse durch öfter auftretende kardiovaskuläre Allgemeinerkrankungen wie beispielsweise Arteriosklerose im Vergleich zu jüngeren Patienten werden diskutiert (Lanzino et al. 1996; Magge et al. 2010). Häufig sind auch Unterschiede in der Definition des Vasospasmus, in der Art der Messung desselben und in der Festlegung der Alterskategorien Faktoren, die einen direkten Vergleich dieser Studien erschweren (Lee et al. 2013).

Unabhängig von diesen Erkenntnissen erzielte die Analyse unserer Daten eine hohe Signifikanz für das Alter als Risikofaktor für einen Vasospasmus. Die Altersgrenze wurde bei 60 Jahren gesetzt und Patienten über 60 Jahre zeigten deutlich weniger Vasospasmen. Sowohl im Chi-Quadrat-Test $(\mathrm{p}<0,0001)$ als auch in der multivariaten Analyse mehrerer Einflussfaktoren ( $p<0,0001)$ erwies sich ein Alter von $\leq 60$ Jahren als deutlich erhöhtes Risiko für die VasospasmusEntwicklung.

\subsubsection{Vasospasmusbehandlung}

Mehrere Wege stehen zur Diagnostik von Vasospasmen zur Verfügung. Neben angiographischen Untersuchungen (CTA, DSA) wird die transkranielle Dopplersonographie (TCD) zur Beurteilung des zerebralen Blutflusses sehr häufig verwendet (Aaslid et al. 1986). Symptomatische Vasospasmen, die mit neurologischen Defiziten durch Ischämien (DIND) einhergehen, werden durch das Auftreten eben solcher Defizite, die nicht in Zusammenhang mit anderen Komplikationen wie z.B. Hydrozephalus, erneuter Blutung, Infektionen oder OP-bezogenen Veränderungen gebracht werden können, diagnostiziert (Torbey et 
al. 2001). Vasospasmus-induzierte Infarkte können zusätzlich per cCT dargestellt werden. Die grundsätzliche Maßnahme zur Behandlung eines Vasospasmus besteht in der Relaxation der betroffenen Gefäße, um eine ausreichende Durchblutung sicherzustellen. Natürlich kommt auch der Prävention eine große Bedeutung $\mathrm{zu}$, sodass bei der initialen aSAB-Behandlung bereits eine Risikominimierung stattfindet. Dies beinhaltet im Falle einer chirurgischen Intervention, dass intraoperativ das subarachnoidal angesammelte Blut(gerinnsel) soweit möglich vollständig entfernt wird und in den Folgetagen (auch durch medikamentöse Unterstützung) die betroffenen Gebiete schnellstmöglich von Blutresten befreit werden, um die umliegenden Gefäße nicht durch freigesetzte Blutbestandteile $\mathrm{zu}$ reizen (Reilly et al. 2004). Angelegte Drainagen (EVD, LD) dienen neben der Liquorableitung zur Verringerung des Hirndrucks bei Hydrozephalus zusätzlich zur Auswaschung von Blut aus dem Liquor.

Darüber hinaus unterläuft das OP-Gebiet während des Clippings regelmäßigen Spülungen mit Nimodipin, einem Calcium-Antagonisten, der eine Gefäßkontraktion gerade im Bereich der durch das Clipping manipulierten Gefäße verhindern soll. Nimodipin wird zusätzlich postoperativ eingesetzt, um Vasospasmen zu behandeln. Die Patienten unserer Studie wurden prophylaktisch in den ersten 14 Tagen nach Operation mit einer standardmäßigen Nimodipingabe $(10 \mathrm{ml} / \mathrm{h}$ intravenös $)$ versorgt. Der positive Effekt von Nimodipin auf Vasospasmen ist seit vielen Jahren bekannt und es erfährt eine breite, auch standardmäßige Anwendung (Barker und Ogilvy 1996; Dorhout Mees et al. 2007).

Bei notwendigen weiterführenden Maßnahmen zum Management von behandlungsbedürftigen Vasospasmen wurde in unserem Fall eine modifizierte Triple- $H$-Therapie angewendet, die hauptsächlich aus einer strengen Hypertension und einer milden Hypervolämie bestand (s. Kapitel 2.4.3). Gerade diese beiden Anteile der Triple-H-Therapie sind seit langem fester Bestandteil der Vasospasmusbehandlung, auch wenn nach wie vor über das Ausmaß und die Häufigkeit des Einsatzes diskutiert wird. So gibt es bisher keinen eindeutigen Konsens darüber, ob beispielsweise eine standardmäßige, prophylaktische oder eine nur symptombezogene Therapie sinnvoll ist, insbesondere auch bei Vorliegen kardiopulmonaler Vorerkrankungen (Findlay et al. 2015; Kassell et al. 1985; Meyer et al. 2011). Die nicht-modifizierte Anwendung der Triple-H-Therapie hat in einigen Fällen zu weiterführenden Komplikationen vor allem im kardiovaskulären 
und pulmonalen Bereich geführt, welche ein Risiko erhöhter Morbidität und Mortalität zur Folge hatten. Insbesondere die induzierte Hypertension und Hypervolämie können bei bereits vorerkrankten, älteren Patienten schwerwiegende Komplikationen wie Lungenödeme, myokardiale Ischämien oder kardiale Arrhythmien hervorrufen (Raabe et al. 2005; Taccone et al. 2009; Zada et al. 2010). Raabe et al. zeigten in ihrer Studie auch, dass Modifizierungen der Triple-H-Therapie wie beispielsweise moderate Hypertension, Hämodilution und Normovolämie zu verringertem Auftreten von Komplikationen im Vergleich zur hypervolämischen Therapie führten (Raabe et al. 2005). Eine frühere Untersuchung kam allerdings zu dem Ergebnis, dass zwischen zwei Gruppen mit und ohne prophylaktische Triple- $H$-Therapie kein signifikanter Unterschied auf das Vasospasmus-Vorkommen oder das Outcome festzustellen war. Dabei kam es aber auch hier in der Gruppe mit Triple-H-Therapie häufiger zu therapiebegleitenden Komplikationen (Egge et al. 2001).

Der Einsatz und der Umfang einzelner Komponenten der Triple- $H$-Therapie sollte dementsprechend immer in Abhängigkeit vom Gesamtzustand des Patienten abgewogen werden, um das Risiko zusätzlicher Komplikationen gering zu halten. In seltenen Fällen fanden neben den aufgeführten konservativen auch invasivere Therapiemethoden wie Ballondilatation betroffener Gefäße oder die intraarterielle Gabe von Nimodipin zur Minderung der Gefäßspasmen Anwendung. Die genannten Therapiebestandteile wurden über den gesamten Studienzeitraum von 1990 bis 2013 nach gleichen Kriterien und Vorgaben eingesetzt.

\subsection{Vasospasmus und temporäres Clipping}

Die grundsätzliche Frage, inwieweit eine (chirurgische) Manipulation der betroffenen Gefäße und deren umgebenden Gewebe Einfluss auf die Entwicklung von Vasospasmen hat, wird seit Längerem diskutiert. Die chirurgische Freilegung der Gefäße und Aneurysmen bringt zwangsläufig eine solche Manipulation mit sich. Auch das Anlegen der definitiven Clips stellt eine Beeinflussung des tragenden Gefäßes dar. Es bleibt zu erörtern, ob durch das Anbringen noch zusätzlicher, temporärer Clips weitere Auswirkungen folgen oder ob die kurzzeitige Clip-Ligatur der behandelten Gefäße keine postoperativen Komplikationen nach sich zieht. 
$\mathrm{Li}$ et al. zeigten beispielsweise in einer umfangreichen Meta-Analyse, dass nach Clipping signifikant häufiger Vasospasmen auftraten als nach Coiling. Allerdings gab es keine signifikanten Unterschiede in Bezug auf postoperative, ischämische Infarkte der operierten Gebiete (Li et al. 2013). Die Untersuchungen von Woertgen et al. lassen den Schluss zu, dass temporäres Clipping mit dazu beitragen kann, dass sich postoperativ Vasospasmen entwickeln, wohingegen auch hier kein signifikanter Einfluss auf die Entwicklung postoperativer Ischämien erkennbar ist (Woertgen et al. 2008). Sowohl eine neuere Studie von Suzuki und Taki als auch eine ältere Meta-Analyse von de Oliveira et al. dagegen kamen zu dem Ergebnis, dass die Wahl der Therapie (Coiling oder Clipping) keine Auswirkung auf das Entstehen von Vasospasmen und deren Folgen wie DIND oder Infarkte hat (de Oliveira et al. 2007; Suzuki und Taki 2013). Häufig wird als Limitation solcher Studien angegeben, dass die Definition und Diagnostik des Vasospasmus eine große Rolle in der Auswertung spielt. So gibt es oft Differenzen in der Auswertung zwischen angiographisch oder dopplersonographisch gemessenen Spasmen oder den als grenzwertig angegebenen Flussgeschwindigkeiten. Andere Autoren registrieren lediglich symptomatische Vasospasmen (DIND) oder im cCT aufgetretene Infarkte. Somit wird eine einheitliche Auswertung und Aussage deutlich erschwert.

Weitere Studien, die speziell auf temporäres Clipping eingehen, untersuchten eher den Einfluss auf das generelle Outcome der Patienten, ohne ein direktes Augenmerk auf Vasospasmen und/oder Infarkte zu legen. Dort zeigte sich, dass, insbesondere bei einer zeitlichen Beschränkung der vorübergehenden Gefäßausschaltung auf 15 bis 20 Minuten, keine signifikante Beeinflussung des Outcomes festzustellen ist. Vor allem bei Patienten mit intraoperativen Aneurysma-Rupturen wird das Outcome sogar eher durch das temporäre Clipping verbessert (Dhandapani et al. 2013; Griessenauer et al. 2014).

In unserer Studie lag der Fokus auf den postoperativ durch TCD gemessenen zerebralen Blutflüssen und den so aufgezeigten Vasospasmen. Der Grenzwert lag in diesem Fall bei $120 \mathrm{~cm} / \mathrm{s}$ mittlerer Flussgeschwindigkeit. Zusätzlich registrierten wir aufgetretene DINDs und Spasmus-induzierte, postoperative Infarkte und werteten diese in Bezug auf stattgefundenes temporäres Clipping aus. In der Analyse der einzelnen Faktoren konnten wir im Gegensatz zu den Studien von Woertgen et al. und $\mathrm{Li}$ et al. keine signifikante Beziehung zwischen 
temporärem Clipping und aufgetretenem Vasospasmus nachweisen. Sowohl in der Gruppe der Patienten ohne (59,3\%) als auch bei Patienten mit temporärem Clipping (63,3 \%) entwickelten sich in ähnlich häufigem Ausmaß Vasospasmen $(\mathrm{p}=0,39)$. Vergleichbare Ergebnisse - ähnlich wie bei den vorangehenden Studien - zeigte die Analyse bei aufgetretenen DINDs (21,5 \% vs. 26,2 \%; p = 0,26). Auffällig in unserer Studie war hingegen das Auftreten Spasmus-induzierter Infarkte bei postoperativen cCT-Aufnahmen. Hier gab es deutlich weniger Infarkte bei Patienten mit temporärem Clipping. 74,3 \% aller Infarkte zeigten sich bei Patienten ohne temporäres Clipping $(\mathrm{p}=0,02)$. Dieses Ergebnis entsprach nicht den vorangegangenen Überlegungen. Die Erwartung lag eher darin, dass es durch die zusätzliche Manipulation der Gefäße mit weiteren Clips auch zu einer erhöhten Gefahr von Infarkten kommt. Eine Möglichkeit zur Erklärung ist, dass sich durch das Anlegen temporärer Clips eine übersichtlichere OP-Situation ergibt, die eine sorgfältigere Präparation der entsprechenden Gefäße und umliegenden Gewebe ermöglicht, sodass eine möglichst geringe Reizung dieser Regionen erfolgt. Eine so durchgeführte, eventuell schonendere Operation könnte zu einer Senkung der postoperativen Infarkt-Rate führen. In dieser Hinsicht wären weiterführende Studien zu genaueren Untersuchung dieses Ergebnisses wünschenswert.

In der multivariaten, logistischen Regressionsanalyse der Einflussfaktoren auf den Vasospasmus untersuchten wir temporäres Clipping als Hauptfaktor. Temporäres Clipping zeigte keinen signifikanten Einfluss darauf, ob postoperativ ein Vasospasmus entsteht oder nicht (OR =0,88; [0,57-1,35] 95\% KI; p =0,84). Somit erwies es sich auch in dieser Hinsicht als eine sichere Behandlungsmethode.

Die Analyse des Outcomes der Patienten nach GOS zeigte wie auch schon vorangehende Studien, dass das temporäre Clipping keine negativen Auswirkungen hat. Der prozentuale Anteil an Patienten mit gutem Behandlungsergebnis (GOS IV - V) war in der Gruppe der mit temporärem Clipping behandelten Aneurysmen sogar etwas höher als in der Vergleichsgruppe ohne temporäres Clipping, allerdings war auch dieser Unterschied nicht signifikant $(59,7 \%$ vs. 64,6 \%; $p=0,19)$. Auch hier zeigte sich, dass der Einsatz temporärer Clips im Zuge der Operation keine nachteilige Auswirkung auf den weiteren Heilungsverlauf des Patienten hatte.

Diese Ergebnisse unserer Studie reihen sich in die Ergebnisse vorhandener Studien wie die von Samson et al., Dhandapani et al. oder Griessenauer et al. ein 
und bestätigen mit einer deutlich höheren Patientenzahl als vorangegangene Studien, dass die Anwendung temporärer Clips keinen negativen Einfluss auf die postoperative Entwicklung, insbesondere auf die Entwicklung von Vasospasmen und das Outcome des Patienten hat und in bestimmten Fällen (wie z.B. einer intraoperativen Ruptur) eher ein besseres Behandlungsergebnis ermöglicht.

\subsection{Limitationen der Studie}

\subsubsection{Datensammlung}

Die für diese Studie ausgewählten Daten wurden retrospektiv über mehrere Jahre zusammengetragen. Die bereits bestehende Datenbank mit den Patienten der Aachener Universitätsklinik wurde übernommen und die Göttinger Patienten nach gleichem Schema in eine neue Datenbank aufgenommen. Aufgrund der retrospektiven Zusammenstellung ist sowohl bei der Aachener als auch bei der Göttinger Datenbank das Problem entstanden, dass nicht zu allen Patienten ein vollständiger Datensatz erhoben werden konnte. Das führte dazu, dass Patienten, zu denen nicht genügend Daten vorhanden waren, nicht in die Analyse mit einbezogen werden konnten. Entscheidende Kriterien waren in diesem Fall Angaben zu GCS, Hunt und Hess-Grad, Fisher-Grad, Art der Intervention (Clipping oder Coiling), Auftreten eines Vasospasmus und GOS. Dadurch reduzierte sich die Patientenzahl der Aachener Datenbank von ursprünglich 758 auf 456. Aus dem Göttinger Patientenkollektiv fanden von 322 Patienten letztlich 299 Eingang in die endgültige Datenbank. Das so entstandene Gesamtkollektiv von 755 Patienten wurde für die hier vorliegende Untersuchung auf die 535 Patienten reduziert, die einer chirurgischen Therapie mit Clipping der Aneurysmen unterzogen wurden. Allerdings ist auch zur vorliegenden Datenbank anzumerken, dass vereinzelt Daten nicht mehr erhoben werden konnten und somit fehlen. Dadurch erklärt sich die variierende Fallzahl der untersuchten Faktoren. Dies ist bei einer retrospektiven Analyse nicht vermeidbar. An dieser Stelle wäre eine prospektive Studie wünschenswert, in der alle nötigen Daten laufend erhoben werden können, sodass eine vollständige Datenbank zur Auswertung der Untersuchung zur Verfügung stünde. 
Darüber hinaus ist es ein weiterer limitierender Faktor, dass zwei Datenbanken aus unterschiedlichen Kliniken verwendet und für dieselbe Untersuchung zusammengesetzt wurden. Diesbezüglich sehen wir allerdings keine allzu große Einschränkung der Studie, da in beiden Kliniken kontinuierlich unter demselben Therapie-Protokoll, welches von Prof. Dr. V. Rohde von der Aachener Klinik an die UMG übertragen wurde, agiert wurde. Die beiden Patientenkollektive sind somit durchaus miteinander vergleichbar.

\subsubsection{Studiendauer}

Der sehr lange Zeitraum der Studie (1990 - 2013) bringt auch eine gewisse Ungenauigkeit der Untersuchung mit sich. Innerhalb dieser Zeit hat sich sowohl auf dem Gebiet der Diagnostik als auch der Therapiemöglichkeiten viel weiterentwickelt, sodass zum Ende des Studienzeitraumes genauere, vielfältigere und auch umfangreichere Untersuchungen möglich waren, beispielsweise durch neuartige Methoden wie die ICG-Angiographie. Dadurch sind unter Umständen auch die diagnostischen Ergebnisse im Vergleich von Studienbeginn zu Studienende beeinflusst worden. Allerdings ist dahingehend festzuhalten, dass die hauptsächlich in der Untersuchung eine Rolle spielenden Faktoren wie Clipping, TCD-Messungen oder cCT-Aufnahmen auch bereits zu Studienbeginn etablierte Methoden gewesen sind und somit eine gewisse Kontinuität auch über einen so langen Studienzeitraum gewährleistet werden konnte.

\subsubsection{Temporäres Clipping}

Das Hauptaugenmerk unserer Studie lag auf dem temporären Clipping. Hierbei wurde für die Clipping-Operationen festgehalten, ob eine zwischenzeitliche Gefäßausschaltung stattgefunden hat oder nicht. Genauere intraoperative Details könnten hier eventuell noch weitere und aussagekräftigere Ergebnisse in der Untersuchung liefern. So wären Informationen über die Verweildauer der temporären Clips oder auch ihre Anzahl sowie die Häufigkeit des Anlegens der Clips innerhalb einer Operation wünschenswert und könnten weiterführende Analysen ermöglichen. Weiterhin könnte die Angabe darüber, ob das Clipping 
aufgrund einer intraoperativen Aneurysmaruptur oder rein zur besseren Gefäßpräparation erfolgte, weitere Erkenntnisse liefern.

In diesen Bereichen standen uns leider nur in unzureichendem Maße Daten zur Verfügung, sodass diese nicht in die Untersuchung mit einbezogen werden konnten.

\subsection{Ausblick}

Zerebrale Vasospasmen und DIND werden auch weiterhin Hauptziele in der Behandlung von aSAB und auch in der wissenschaftlichen Untersuchung bleiben. Wirksame Konzepte zum Management dieser schwerwiegenden Komplikation sind nach wie vor Gegenstand ständiger Weiterentwicklung und Analyse. Unsere Studie leistet in diesem Zusammenhang mit einem sehr großen Patientenkollektiv und einer umfangreichen Datensammlung über einen langen Zeitraum einen Beitrag in Bezug auf die Art der Intervention und deren mögliche Beeinflussung des postoperativen Verlaufes. Weiterführende Studien wären auch in diesem Bereich wünschenswert, beispielsweise mit prospektiv gesammelten Daten und genaueren intra- und postoperativen Details bezüglich des Clippings und der Vasospasmusdiagnostik, um weitere Einflussfaktoren auf die Spasmusentwicklung noch genauer analysieren zu können. 


\section{Zusammenfassung}

Der Behandlung von aneurysmatischen Subarachnoidalblutungen kommt aufgrund der Schwere der Erkrankung, verbunden mit hoher Mortalität und Morbidität, nach wie vor eine hohe Bedeutung im Bereich der neurologischen Krankheitsbilder zu. Die Vor- und Nachteile verschiedener Therapie-Möglichkeiten wie Coiling, Clipping oder auch temporäres Clipping werden immer wieder diskutiert. Auch die Behandlung der mit der Erkankung einhergehenden Komplikationen, insbesondere der Vasospasmen, steht im Fokus der Diskussion und therapeutischen Weiterentwicklung, sind sie doch einer der Hauptgründe für die blutungsassoziierte Mortalität und Morbidität.

Für diese Arbeit wurde eine retrospektive Datenbank angefertigt, in der alle Patienten, die im Zeitraum von 1990 - 2005 in der Neurochirurgischen Universitätsklinik der RWTH Aachen und von 2005 - 2013 in der Abteilung für Neurochirurgie der Universitätsmedizin Göttingen mit aSAB behandelt worden sind, zusammengetragen wurden. Aus diesem Patientenkollektiv wurden alle Patienten mit chirurgischem Aneurysmaverschluss (Clipping) in einer statistischen Untersuchung analysiert. Hauptziel der Analyse war der Einfluss einer intraoperativen temporären Gefäßausschaltung auf das Auftreten postoperativer Vasospasmen.

In unserer Untersuchung konnte gezeigt werden, dass das temporäre Clipping der Gefäße keinen signifikanten Einfluss auf die postoperative Entwicklung, speziell auf die Häufigkeit der Vasospasmen, hat. Andere Faktoren, wie die Schwere und Ausdehnung der aSAB im Vorfeld der Operation und das Alter des Patienten wirken sich, wie in vorhergehenden Studien auch gezeigt, durchaus auf das Vasospasmus-Aufkommen aus. Bei schweren und ausgedehnten Blutungen treten häufiger Vasospasmen auf, ebenso bei Patienten unter 60 Jahren. Auch in Zukunft wird das Interesse daran hoch bleiben, die Einflussfaktoren und die Behandlungsmöglichkeiten des Vasospasmus $\mathrm{zu}$ untersuchen und durch verbesserte Therapien die dadurch bedingte Mortalitäts- und Morbiditätsrate zu senken.

Die Operationsmethode des temporären Clippings erwies sich in unserer Untersuchung dagegen, wie schon zuvor in anderen Studien zu ähnlicher Thematik, 
als sichere Behandlung bei der chirurgischen Aneurysmaversorgung ohne negativen Einfluss auf den postoperativen Verlauf und das Outcome des Patienten. 


\section{Literaturverzeichnis}

Aaslid R, Markwalder TM, Nornes H (1982): Noninvasive transcranial Doppler ultrasound recording of flow velocity in basal cerebral arteries. J Neurosurg $\underline{57}, 769-774$

Aaslid R, Huber P, Nornes H (1984): Evaluation of cerebrovascular spasm with transcranial Doppler ultrasound. J Neurosurg $\underline{60}, 37-41$

Aaslid R, Huber P, Nornes H (1986): A transcranial Doppler method in the evaluation of cerebrovascular spasm. Neuroradiology $\underline{28}, 11-16$

Ammerer HP, Dobner T, Ive-Schappelwein H, Loyoddin M (2003): Die Behandlung intrakranieller Aneurysmen - eine Herausforderung im Wandel der Zeit. J Neurol Neurosurg Psychiatry $4,14-$ 21

Barker FG, Ogilvy CS (1996): Efficacy of prophylactic nimodipine for delayed ischemic deficit after subarachnoid hemorrhage: a metaanalysis. J Neurosurg $\underline{84}, 405-414$

Batjer H, Samson D (1986): Intraoperative aneurysmal rupture: incidence, outcome, and suggestions for surgical management. Neurosurgery $\underline{18}, 701-707$

Biller J, Godersky JC, Adams HP (1988): Management of aneurysmal subarachnoid hemorrhage. Stroke $19,1300-1305$

Boecher-Schwarz HG, Ungersboeck K, Ulrich P, Fries G, Wild A, Perneczky A (1994): Transcranial Doppler diagnosis of cerebral vasospasm following subarachnoid haemorrhage: Correlation and analysis of results in relation to the age of patients. Acta Neurochir $\underline{127}, 32-36$

Bor ASE, Rinkel GJE, Adami J, Koffijberg H, Ekbom A, Buskens E, Blomqvist P, Granath F (2008): Risk of subarachnoid haemorrhage according to number of affected relatives: a population based case-control study. Brain 131, 2662-2665

Bor ASE, Rinkel GJE, van Norden J, Wermer MJH (2014): Long-term, serial screening for intracranial aneurysms in individuals with a family history of aneurysmal subarachnoid haemorrhage: a cohort study: A cohort study. Lancet Neurol 13, 385-392

Charbel FT, Ausman JI, Diaz FG, Malik GM, Dujovny M, Sanders J (1991): Temporary clipping in aneurysm surgery: Technique and results. Surg Neurol $\underline{36}, 83-90$

Charpentier C, Audibert G, Guillemin F, Civit T, Ducrocq X, Bracard S, Hepner H, Picard L, Laxenaire MC (1999): Multivariate Analysis of Predictors of Cerebral Vasospasm Occurrence After Aneurysmal Subarachnoid Hemorrhage. Stroke 30, 1402-1408

Cossu G, Messerer M, Oddo M, Daniel RT (2014): To look beyond vasospasm in aneurysmal subarachnoid haemorrhage. Biomed Res Int 2014, 628597 
Crowley RW, Medel R, Dumont AS, Ilodigwe D, Kassell NF, Mayer SA, Ruefenacht D, Schmiedek P, Weidauer S, Pasqualin A (2011): Angiographic vasospasm is strongly correlated with cerebral infarction after subarachnoid hemorrhage. Stroke $\underline{42}$, 919-923

de Oliveira JG, Beck J, Ulrich C, Rathert J, Raabe A, Seifert V (2007): Comparison between clipping and coiling on the incidence of cerebral vasospasm after aneurysmal subarachnoid hemorrhage: a systematic review and meta-analysis. Neurosurg Rev $\underline{30}, 22-30$

Dhandapani S, Pal SS, Gupta SK, Mohindra S, Chhabra R, Malhotra SK (2013): Does the impact of elective temporary clipping on intraoperative rupture really influence neurological outcome after surgery for ruptured anterior circulation aneurysms? - A prospective multivariate study. Acta Neurochir $\underline{155}, 237-246$

Dorhout Mees SM, Rinkel GJE, Feigin VL, Algra A, van den Bergh, W M, Vermeulen M, van Gijn J (2007): Calcium antagonists for aneurysmal subarachnoid haemorrhage. Cochrane Database Syst Rev, CD000277

Dorsch NW, King MT (1994): A review of cerebral vasospasm in aneurysmal subarachnoid haemorrhage Part I: Incidence and effects. J Clin Neurosci 1, 19-26

Drake RL, Bräuer L, Gray H: Gray's Atlas der Anatomie. Elsevier Urban \& Fischer, München 2013

Dumont AS, Lanzino G, Kassell NF (2002): Unruptured aneurysms. J Neurosurg 96, 52-56

Dumont AS, Crowley RW, Monteith SJ, Ilodigwe D, Kassell NF, Mayer S, Ruefenacht D, Weidauer S, Pasqualin A, Macdonald RL (2010): Endovascular treatment or neurosurgical clipping of ruptured intracranial aneurysms: effect on angiographic vasospasm, delayed ischemic neurological deficit, cerebral infarction, and clinical outcome. Stroke $\underline{41}, 2519-2524$

D'Urso PI, Lanzino G, Cloft HJ, Kallmes DF (2011): Flow diversion for intracranial aneurysms: a review. Stroke $\underline{42}, 2363-2368$

Edlow JA, Caplan LR (2000): Avoiding pitfalls in the diagnosis of subarachnoid hemorrhage. N Engl J Med $\underline{342}, 29-36$

Egge A, Waterloo K, Sjoholm H, Solberg T, Ingebrigtsen T, Romner B (2001): Prophylactic hyperdynamic postoperative fluid therapy after aneurysmal subarachnoid hemorrhage: a clinical, prospective, randomized, controlled study. Neurosurgery 49 , 593-605

Etminan N, Brown RD, Beseoglu K, Juvela S, Raymond J, Morita A, Torner JC, Derdeyn CP, Raabe A, Mocco J (2015): The unruptured intracranial aneurysm treatment score: A multidisciplinary consensus. Neurology $\underline{85}, 881-889$

Feigin VL, Rinkel GJE, Lawes CMM, Algra A, Bennett DA, van Gijn J, Anderson CS (2005): Risk factors for subarachnoid hemorrhage: an updated systematic review of epidemiological studies. Stroke $\underline{36}, 2773-2780$

Findlay JM, Nisar J, Darsaut T (2015): Cerebral Vasospasm: A Review. (Can J Neurol Sci, im Druck) 
Fisher CM, Kistler JP, Davis JM (1980): Relation of cerebral vasospasm to subarachnoid hemorrhage visualized by computerized tomographic scanning. Neurosurgery $\underline{6}, 1-9$

Frontera JA, Fernandez A, Schmidt JM, Claassen J, Wartenberg KE, Badjatia N, Connolly ES, Mayer SA (2009): Defining vasospasm after subarachnoid hemorrhage: what is the most clinically relevant definition? Stroke $\underline{40}, 1963-1968$

Gonzalez NR, Boscardin WJ, Glenn T, Vinuela F, Martin NA (2007): Vasospasm probability index: a combination of transcranial doppler velocities, cerebral blood flow, and clinical risk factors to predict cerebral vasospasm after aneurysmal subarachnoid hemorrhage. J Neurosurg $\underline{107}$, 1101-1112

Greenberg MS: Handbook of neurosurgery. 5. Auflage; Thieme, Stuttgart, New York 2001

Griessenauer CJ, Poston TL, Shoja MM, Mortazavi MM, Falola M, Tubbs RS, Fisher WS (2014): The impact of temporary artery occlusion during intracranial aneurysm surgery on long-term clinical outcome: part I. Patients with subarachnoid hemorrhage. World Neurosurg $\underline{82}$, 140148

Guglielmi G, Vinuela F, Dion J, Duckwiler G (1991): Electrothrombosis of saccular aneurysms via endovascular approach. Part 2: Preliminary clinical experience. J Neurosurg $\underline{75}$ 8-14

Harrington DP, Boxt LM, Murray PD (1982): Digital subtraction angiography: overview of technical principles. AJR Am J Roentgenol 139, 781-786

Hoh BL, Topcuoglu MA, Singhal AB, Pryor JC, Rabinov JD, Rordorf GA, Carter BS, Ogilvy CS (2004): Effect of Clipping, Craniotomy, or Intravascular Coiling on Cerebral Vasospasm and Patient Outcome after Aneurysmal Subarachnoid Hemorrhage. Neurosurgery $\underline{55}$, 779-789

Hunt WE, Hess RM (1968): Surgical risk as related to time of intervention in the repair of intracranial aneurysms. J Neurosurg 28, 14-20

Inagawa T, Yahara K, Ohbayashi N (2014): Risk factors associated with cerebral vasospasm following aneurysmal subarachnoid hemorrhage. Neurol Med Chir (Tokyo) 54, 465-473

International Study of Unruptured Intracranial Aneurysms Investigators (1998): Unruptured intracranial aneurysms - risk of rupture and risks of surgical intervention. N Engl J Med $\underline{339}$, $1725-1733$

Isaksen J, Egge A, Waterloo K, Romner B, Ingebrigtsen T (2002): Risk factors for aneurysmal subarachnoid haemorrhage: the Tromsø study. J Neurol Neurosurg Psychiatr $\underline{73}, 185-187$

Jennett B, Bond M (1975): Assessment of outcome after severe brain damage. Lancet 1 , 480-484

Jones J, Sayre J, Chang R, Tian J, Szeder V, Gonzalez N, Jahan R, Vinuela F, Duckwiler G, Tateshima S (2015): Cerebral vasospasm patterns following aneurysmal subarachnoid hemorrhage: an angiographic study comparing coils with clips. J Neurointerv Surg $\underline{7}, 803-807$

Juvela S, Poussa K, Lehto H, Porras M (2013): Natural history of unruptured intracranial aneurysms: a long-term follow-up study. Stroke $\underline{44}$, 2414-2421 
Kanamaru K, Suzuki H, Taki W (2015): Risk factors for vasospasm-induced cerebral infarct when both clipping and coiling are equally available. Acta Neurochir Suppl 120, 291-295

Kassell NF, Sasaki T, Colohan AR, Nazar G (1985): Cerebral vasospasm following aneurysmal subarachnoid hemorrhage. Stroke $\underline{16}, 562-572$

Kassell NF, Torner JC, Haley EC, Jane JA, Adams HP, Kongable GL (1990a): The International Cooperative Study on the Timing of Aneurysm Surgery. Part 1: Overall management results. J Neurosurg $\underline{73}, 18-36$

Kassell NF, Torner JC, Jane JA, Haley EC, Adams HP (1990b): The International Cooperative Study on the Timing of Aneurysm Surgery. Part 2: Surgical results. J Neurosurg $\underline{73}, 37-47$

Krex D, Schackert HK, Schackert G (2001): Genesis of cerebral aneurysms - an update. Acta Neurochir (Wien) $\underline{143}, 429-448$

Lanzino G, Kassell NF, Germanson TP, Kongable GL, Truskowski LL, Torner JC, Jane JA (1996): Age and outcome after aneurysmal subarachnoid hemorrhage: why do older patients fare worse? J Neurosurg $\underline{85}, 410-418$

Leblanc R (1987): The minor leak preceding subarachnoid hemorrhage. J Neurosurg $\underline{66}$, 35-39

Lee Y, Zuckerman SL, Mocco J (2013): Current controversies in the prediction, diagnosis, and management of cerebral vasospasm: where do we stand? Neurol Res Int 2013, 373458

Leipzig TJ, Morgan J, Horner TG, Payner T, Redelman K, Johnson CS (2005): Analysis of Intraoperative Rupture in the Surgical Treatment of 1694 Saccular Aneurysms. Neurosurgery 56, $455-468$

Li H, Pan R, Wang H, Rong X, Yin Z, Milgrom DP, Shi X, Tang Y, Peng Y (2013): Clipping versus coiling for ruptured intracranial aneurysms: a systematic review and meta-analysis. Stroke $\underline{44}$, 29-37

Lin C, Dumont AS, Zhang JH, Zuccarello M, Muroi C (2014): Cerebral vasospasm after aneurysmal subarachnoid hemorrhage: mechanism and therapies. Biomed Res Int 2014, 679014

Linn FHH, Rinkel GJE, Algra A, van Gijn J (1996): Incidence of Subarachnoid Hemorrhage: Role of Region, Year, and Rate of Computed Tomography: A Meta-Analysis. Stroke 27, 625-629

Linn FHH, Rinkel GJE, Algra A, van Gijn J (1998): Headache characteristics in subarachnoid haemorrhage and benign thunderclap headache. J Neurol Neurosurg Psychiatry $\underline{65}, 791-793$

Macdonald RL, Rosengart AJ, Huo D, Karrison T (2003): Factors associated with the development of vasospasm after planned surgical treatment of aneurysmal subarachnoid hemorrhage. J Neurosurg 99, 644-652

Magge SN, Chen HI, Ramakrishna R, Cen L, Chen Z, Elliott JP, Winn HR, Le Roux PD (2010): Association of a younger age with an increased risk of angiographic and symptomatic vasospasms following subarachnoid hemorrhage. J Neurosurg $\underline{112}$, 1208-1215 
McDougall CG, Spetzler RF, Zabramski JM, Partovi S, Hills NK, Nakaji P, Albuquerque FC (2012): The Barrow Ruptured Aneurysm Trial. J Neurosurg 116, 135-144

Meyer R, Deem S, Yanez ND, Souter M, Lam A, Treggiari MM (2011): Current practices of triple-H prophylaxis and therapy in patients with subarachnoid hemorrhage. Neurocrit Care 14, 24-36

Mielke D, Malinova V, Rohde V (2014): Comparison of intraoperative microscopic and endoscopic ICG angiography in aneurysm surgery. Neurosurgery 10 Suppl 3, 418-425

Mindea SA, Yang BP, Bendok BR, Miller JW, Batjer HH (2006): Endovascular treatment strategies for cerebral vasospasm. Neurosurg Focus 21, E13

Molyneux AJ, Kerr RSC, Yu L, Clarke M, Sneade M, Yarnold JA, Sandercock P (2005): International subarachnoid aneurysm trial (ISAT) of neurosurgical clipping versus endovascular coiling in 2143 patients with ruptured intracranial aneurysms: A randomised comparison of effects on survival, dependency, seizures, rebleeding, subgroups, and aneurysm occlusion. Lancet $\underline{366}$, 809-817

Moskopp D, Wassmann H: Neurochirurgie: Handbuch für die Weiterbildung und interdisziplinäres Nachschlagewerk. Schattauer, Stuttgart 2005

Pluta RM, Hansen-Schwartz J, Dreier J, Vajkoczy P, Macdonald RL, Nishizawa S, Kasuya H, Wellman G, Keller E, Zauner A (2009): Cerebral vasospasm following subarachnoid hemorrhage: time for a new world of thought. Neurol Res $\underline{31}, 151-158$

Pobereskin LH (2001): Incidence and outcome of subarachnoid haemorrhage: A retrospective population based study. J Neurol Neurosurg Psychiatry 70, 340-343

Pool JL (1961): Aneurysms of the anterior communicating artery. Bifrontal craniotomy and routine use of temporary clips. J Neurosurg $\underline{18}, 98-112$

Raabe A, Beck J, Keller M, Vatter H, Zimmermann M, Seifert V (2005): Relative importance of hypertension compared with hypervolemia for increasing cerebral oxygenation in patients with cerebral vasospasm after subarachnoid hemorrhage. J Neurosurg $\underline{103}, 974-981$

Rabb CH, Tang G, Chin LS, Giannotta SL (1994): A statistical analysis of factors related to symptomatic cerebral vasospasm. Acta Neurochir $\underline{127}, 27-31$

Reilly C, Amidei C, Tolentino J, Jahromi BS, Macdonald RL (2004): Clot volume and clearance rate as independent predictors of vasospasm after aneurysmal subarachnoid hemorrhage. J Neurosurg $\underline{101}, 255-261$

Rinkel GJE, Djibuti M, Algra A, van Gijn J (1998): Prevalence and Risk of Rupture of Intracranial Aneurysms: A Systematic Review. Stroke 29, 251-256

Rooij NK de, Linn FHH, van der Plas, J A, Algra A, Rinkel GJE (2007): Incidence of subarachnoid haemorrhage: a systematic review with emphasis on region, age, gender and time trends. J Neurol Neurosurg Psychiatr 78, 1365-1372 
Sacco RL, Wolf PA, Bharucha NE, Meeks SL, Kannel WB, Charette LJ, McNamara PM, Palmer EP, D'Agostino R (1984): Subarachnoid and intracerebral hemorrhage: natural history, prognosis, and precursive factors in the Framingham Study. Neurology $\underline{34}$, 847-854

Samson D, Batjer HH, Bowman G, Mootz L, Krippner WJ, JR, Meyer YJ, Allen BC (1994): A clinical study of the parameters and effects of temporary arterial occlusion in the management of intracranial aneurysms. Neurosurgery $\underline{34}, 22-28$

Sandström N, Yan B, Dowling R, Laidlaw J, Mitchell P (2013): Comparison of microsurgery and endovascular treatment on clinical outcome following poor-grade subarachnoid hemorrhage. J Clin Neurosci 20, 1213-1218

Schievink WI, Michels VV, Piepgras DG (1994): Neurovascular manifestations of heritable connective tissue disorders. A review. Stroke 25, 889-903

Schievink WI (1997a): Genetics of intracranial aneurysms. Neurosurgery $\underline{40}$ 651-662

Schievink WI (1997b): Intracranial aneurysms. N Eng J Med 336, 28-40

Schramm J, Cedzich C (1993): Outcome and management of intraoperative aneurysm rupture. Surg Neurol 40 , 26-30

Siasios I, Kapsalaki EZ, Fountas KN (2012): The role of intraoperative micro-Doppler ultrasound in verifying proper clip placement in intracranial aneurysm surgery. Neuroradiology $\underline{54}, 1109$ 1118

Spetzler RF, McDougall CG, Zabramski JM, Albuquerque FC, Hills NK, Russin JJ, Partovi S, Nakaji P, Wallace RC (2015): The Barrow Ruptured Aneurysm Trial: 6-year results. J Neurosurg $\underline{123}$, 609-617

Suarez JI, Tarr RW, Selman WR (2006): Aneurysmal subarachnoid hemorrhage. N Engl J Med $\underline{354}$ 387-396

Sudlow C, Warlow CP (1997): Comparable Studies of the Incidence of Stroke and its Pathological Types: Results From an International Collaboration. Stroke 28, 491-499

Suzuki H, Taki W (2013): Effect of aneurysm treatment modalities on cerebral vasospasm after aneurysmal subarachnoid hemorrhage. Acta Neurochir Suppl 115, 99-105

Suzuki J, Kwak R, Okudaira Y (1979): The safe time limit of temporary clamping of cerebral arteries in the direct surgical treatment of intracranial aneurysm under moderate hypothermia. Tohoku J Exp Med 127, 1-7

Taccone FS, Lubicz B, Piagnerelli M, van Nuffelen M, Vincent J, Backer D de (2009): Cardiogenic shock with stunned myocardium during triple-H therapy treated with intra-aortic balloon pump counterpulsation. Neurocrit Care $\underline{10}$ 76-82

Taylor CL, Yuan Z, Selman WR, Ratcheson RA, Rimm AA (1995): Cerebral arterial aneurysm formation and rupture in 20,767 elderly patients: hypertension and other risk factors. J Neurosurg $\underline{83}, 812-819$ 
Taylor CL, Selman WR, Kiefer SP, Ratcheson RA, Ratcheson RA (1996): Temporary vessel occlusion during intracranial aneurysm repair. Neurosurgery $\underline{39}$, 893-905

Teasdale G, Jennett B (1974): Assessment of Coma and Impaired Consciousness. Lancet 304, 81-84

Teasdale GM, Drake CG, Hunt WE, Kassell NF, Sano K, Pertuiset B, Villiers JC de (1988): A universal subarachnoid hemorrhage scale: Report of a committee of the World Federation of Neurosurgical Societies. J Neurol Neurosurg Psychiatry $\underline{51}, 1457$

Teunissen LL, Rinkel GJ, Algra A, van Gijn J (1996): Risk factors for subarachnoid hemorrhage: a systematic review: A Systematic Review. Stroke 27, 544-549

Torbey MT, Hauser TK, Bhardwaj A, Williams MA, Ulatowski JA, Mirski MA, Razumovsky AY (2001): Effect of age on cerebral blood flow velocity and incidence of vasospasm after aneurysmal subarachnoid hemorrhage. Stroke $\underline{32}$, 2005-2011

Treggiari-Venzi MM, Suter PM, Romand JA (2001): Review of medical prevention of vasospasm after aneurysmal subarachnoid hemorrhage: a problem of neurointensive care. Neurosurgery 48, 249-261

van der Schaaf I, Algra A, Wermer M, Molyneux AJ, Clarke M, van Gijn J, Rinkel GJE (2005): Endovascular coiling versus neurosurgical clipping for patients with aneurysmal subarachnoid haemorrhage. Cochrane Database Syst Rev, CD003085

van Gijn J, Rinkel GJE (2001): Subarachnoid haemorrhage: diagnosis, causes and management. Brain $\underline{124}, 249-278$

van Gijn J, Kerr RSC, Rinkel GJE (2007): Subarachnoid haemorrhage. Lancet $\underline{369}$, 306-318

Vlak MH, Algra A, Brandenburg R, Rinkel GJE (2011): Prevalence of unruptured intracranial aneurysms, with emphasis on sex, age, comorbidity, country, and time period: A systematic review and meta-analysis. Lancet Neurol 10, 626-636

Wachter D, Kreitschmann-Andermahr I, Gilsbach JM, Rohde V (2011a): Early surgery of multiple versus single aneurysms after subarachnoid hemorrhage: an increased risk for cerebral vasospasm? J Neurosurg 114, 935-941

Wachter D, Hans F, Kreitschmann-Andermahr I, Rohde V (2011b): Lower incidence of transcranial Doppler and symptomatic vasospasm after aneurysmal subarachnoid hemorrhage and aneurysm clipping in the elderly patient? Neurosurgery $\underline{69}, 261-266$

Washington CW, Zipfel GJ, Chicoine MR, Derdeyn CP, Rich KM, Moran CJ, Cross DT, Dacey RG (2013): Comparing indocyanine green videoangiography to the gold standard of intraoperative digital subtraction angiography used in aneurysm surgery. J Neurosurg $\underline{118}$ $420-427$

Weir B, Grace M, Hansen J, Rothberg C (1978): Time course of vasospasm in man. J Neurosurg $\underline{48}$ 173-178

Weir B (1995): The pathophysiology of cerebral vasospasm. Br J Neurosurg $\underline{9}$, 375-390 
Weir BK, Kongable GL, Kassell NF, Schultz JR, Truskowski LL, Sigrest A (1998): Cigarette smoking as a cause of aneurysmal subarachnoid hemorrhage and risk for vasospasm: a report of the Cooperative Aneurysm Study. J Neurosurg $\underline{89}, 405-411$

Wiebers DO, Whisnant JP, Sundt TM, O'Fallon WM (1987): The significance of unruptured intracranial saccular aneurysms. J Neurosurg $\underline{66}, 23-29$

Wiebers DO, Whisnant JP, Huston J, Meissner I, Brown RD, Piepgras DG, Forbes GS, Thielen K, Nichols D, O'Fallon WM (2003): Unruptured intracranial aneurysms: natural history, clinical outcome, and risks of surgical and endovascular treatment. Lancet $\underline{362}$, 103-110

Winn HR, Jane JA, SR, Taylor J, Kaiser D, Britz GW (2002): Prevalence of asymptomatic incidental aneurysms: review of 4568 arteriograms. J Neurosurg $\underline{96}, 43-49$

Woertgen C, Rothoerl RD, Albert R, Schebesch K, Ullrich O (2008): Effects of temporary clipping during aneurysm surgery. Neurol Res $\underline{30}$ 542-546

Zada G, Terterov S, Russin J, Clavijo L, Giannotta S (2010): Cerebral vasospasm and concurrent left ventricular outflow tract obstruction: requirement for modification of hyperdynamic therapy regimen. Neurocrit Care 12, 265-268 


\section{Danksagungen}

Mein herzlicher Dank gilt zuallererst Frau Prof. Dr. Dorothee Mielke, geschäftsführende Oberärztin der Klinik für Neurochirurgie, Universitätsmedizin Göttingen, für die Überlassung des Themas der vorliegenden Dissertation und die durchgehend engagierte und unterstützende Betreuung. Vielen Dank für ein stets offenes Ohr und die gute Hilfe bei jeder Art von Fragen!

Den Mitarbeitern des Instituts für Medizinische Statistik der Universitätsmedizin Göttingen möchte ich für die Beratungen und Hilfestellungen $\mathrm{zu}$ statistischen Berechnungen und Auswertungen im Rahmen des „Betreuten Arbeitens“ in ihrem Institut danken.

Meiner Studiums- und Doktorandenkollegin Patricia Suntheim gebührt ein großer Dank für die tolle Zusammenarbeit während der gesamten Zeit, für alle Hilfe und den guten Austausch von Ideen und für die zahlreichen gemeinsamen Arbeitsstunden im Archiv. Danke!

Zu guter Letzt geht mein Dank an meine Schwester und in besonderem Maße an meine Mutter, für das gründliche Korrekturlesen, aber auch - und vor allem - für die immerwährende Unterstützung und Motivation über all die Jahre. Vielen, herzlichen Dank. 